\title{
Development of computational tools for modeling engine fuel economy and emissions
}

Christopher L. Marlowe
West Virginia University

West Virginia University

Follow this and additional works at: https://researchrepository.wvu.edu/etd

\section{Recommended Citation}

Marlowe, Christopher L., "Development of computational tools for modeling engine fuel economy and emissions" (2009). Graduate Theses, Dissertations, and Problem Reports. 2060.

https://researchrepository.wvu.edu/etd/2060

This Thesis is protected by copyright and/or related rights. It has been brought to you by the The Research Repository @ WVU with permission from the rights-holder(s). You are free to use this Thesis in any way that is permitted by the copyright and related rights legislation that applies to your use. For other uses you must obtain permission from the rights-holder(s) directly, unless additional rights are indicated by a Creative Commons license in the record and/ or on the work itself. This Thesis has been accepted for inclusion in WVU Graduate Theses, Dissertations, and Problem Reports collection by an authorized administrator of The Research Repository @ WVU. For more information, please contact researchrepository@mail.wvu.edu. 


\title{
Development of Computational Tools for Modeling Engine Fuel Economy and Emissions
}

\author{
Christopher L. Marlowe
}

Thesis submitted to the College of Engineering and Mineral Resources At West Virginia University

In partial fulfillment of the requirements for the degree of

Master of Science
In
Mechanical Engineering

Mario Perhinschi, Ph.D., Chair

Nigel Clark, Ph.D.

Scott Wayne, Ph.D.

Department of Mechanical and Aerospace Engineering

Morgantown, West Virginia

2009

Keywords: Emissions Modeling, Genetic Algorithm, Multivariable Regression, IBIS 


\begin{abstract}
Development of Computational Tools for Modeling Engine Fuel Economy and Emissions Christopher L. Marlowe
\end{abstract}

The Integrated Bus Information System (IBIS) is a vehicle fleet emission and fuel economy prediction software. IBIS is under development by faculty and students of West Virginia University (WVU). The overall goal of IBIS is to provide an approachable and reliable method for users, primarily transit agencies, to evaluate overall fleet emissions and fuel consumption. This approach differs from current modeling packages as IBIS is an online tool and allows for a customizable, user-defined vehicle fleet.

The modeling strategy for IBIS involves creating models using data obtained from the WVU Center for Alternative Fuels, Engines, and Emissions (CAFEE) testing database. These models are multiple variable polynomials created through regression analysis. Additionally, multiplicative and additive correction factors are computed and applied to backbone models to account for variances in vehicle configurations and technologies.

This modeling strategy includes the necessary development of tools to aid in the creation of continuous models. The first to be implemented is a polynomial regression tool. This methodology utilizes data gleaned from the WVU Center for Alternative Fuels, Engines and Emission database. The tool is designed to perform multivariable regression for standard driving cycles: where second-by-second data is available.

The accuracy of these models is reliant upon large sets of data. Furthermore, in cases where limited a dataset is available, additional information may be computed by concatenating experimental data isolated from within existing testing cycles for which testing has been preformed. This data is extracted from a driving cycle by defining periods of non-idle. These periods, or microtrips, are rearranged into new cycles of varying length by a second computational tool.

This second tool is a driving cycle generator which utilizes a genetic algorithm to reorder and concatenate microtrips such that the resulting cycle fulfills criteria supplied by the user. These parameters align with input parameters defining a driving cycle for both IBIS and the polynomial tool: parameters include average speed with idle, standard deviation of speed with idle, kinetic intensity, percentage idle, and number of stops per mile. In addition to providing additional data, the cycle generator yields insight as to acceptable limits on the user inputs defining a driving cycle.

Once the data set has been expanded by the cycle generator, the new data is reintroduced to the polynomial regression tool. Expansion of the data set allows the polynomial tool to 
generate a much more realistic trend for a domain of average speed than was previously obtained with limited data. With the integration of the cycle generator into the polynomial tool, adverse effects caused by interpolation are significantly minimized in the polynomial model.

The use of the polynomial tool has improved and accelerated the design process for models for IBIS. Additionally, the integration of the newly generated cycles through the use of a GA allows for accurate expansion of experimental data without necessitating supplementary dynamometer testing. 


\section{Acknowledgements}

Firstly, I would like to thank Dr. Mario Perhinschi for his advice and support throughout my academic career. I have learned so much under your tutelage. I would never have been able to write computer code and utilize AI techniques with such proficiency were it not for your instruction. My time under your counsel will be permanently etched in my memory, and I will be forever indebted to you.

I would also like to thank my committee members Drs. Scott Wayne and Nigel Clark for their guidance and their unwavering ability to not accept an easy answer.

Special thanks to the US Department of Transportation for the funding and opportunity to be a part of this research effort.

Additionally, I would like to thank Sergio Tamayo for breaking each piece of my software numerous times. Also, Jun Tu for his technical knowledge of the CAFEE testing procedure.

Finally, I would like to thank my family for their unwavering support, and my wife for harassing me to finish my thesis. I have never felt a lack of support, and to this I give you my utmost thanks. 


\section{Table of Contents}

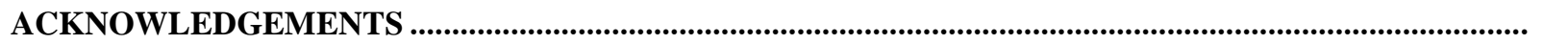

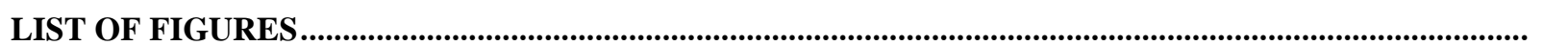

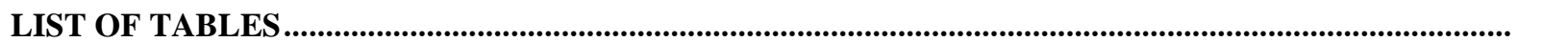

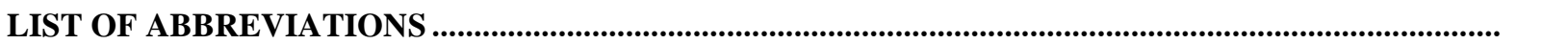

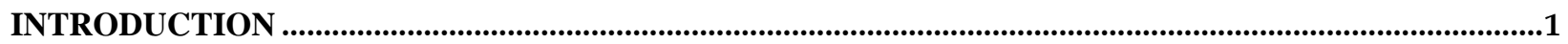

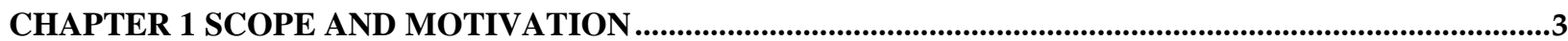

1.1 OVERVIEW OF CURRENT STATE OF THE ART Modeling OF ENGINE Fuel ECONOMY AND EMISSIONS ...............4

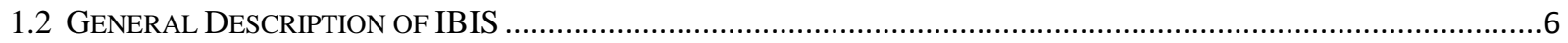

1.3 ANALYSiS of COMPUTATIONAL NEEDS, ReQUiREMENTS, AVAILABLE, AND DEVELOPED METHODOLOGIES......7

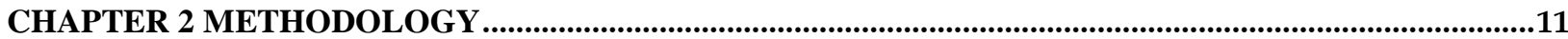

2.1 GENERAL DESCRIPTION OF THE INTERACTIVE COMPUTATIONAL ENVIRONMENT FOR MODELING ENGINE FUEL

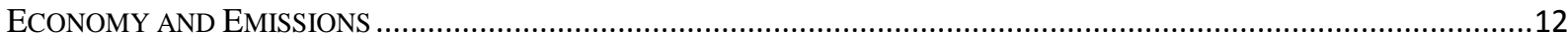

2.2 PolynOMIAL MOdELING BASED ON LINEAR REGRESSION- THEORETICAL BACKGROUND............................13

2.3 GENETIC ALGORITHM OPTIMIZATION- THEORETICAL BACKGROUND .......................................................16

CHAPTER 3 INTERACTIVE COMPUTATIONAL TOOL FOR POLYNOMIAL MODELING OF ENGINE FUEL ECONOMY AND EMISSIONS BASED ON LINEAR REGRESSION ....................................20

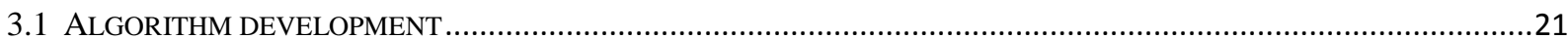

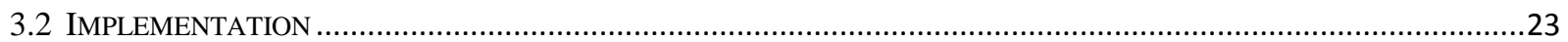

3.3 BACKBONE MODEL DEVELOPMENT USING THE POLYNOMIAL REGRESSION TOOL ........................................26

CHAPTER 4 INTERACTIVE COMPUTATIONAL TOOL FOR DRIVING CYCLE GENERATION BASED ON GENETIC ALGORITHM OPTIMIZATION ............................................................................31

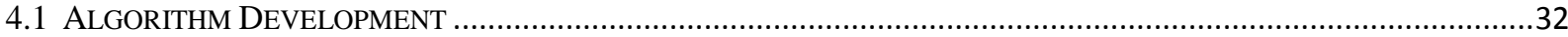

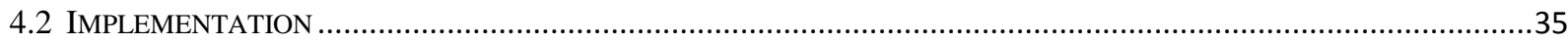

4.3 DEVELOPMENT OF DRIVING CYCLES USING THE CYCLE GENERATOR TOOL ..................................................45

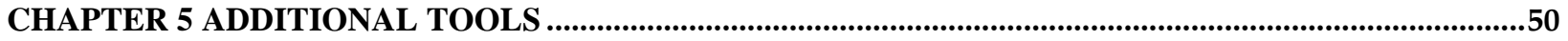

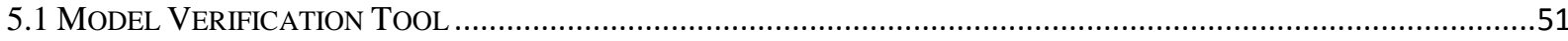

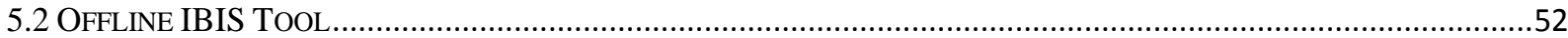

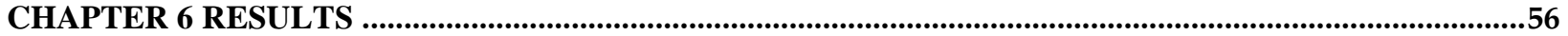

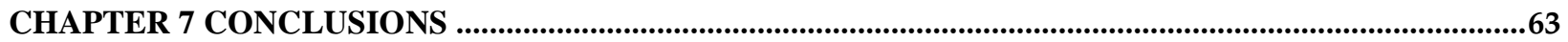

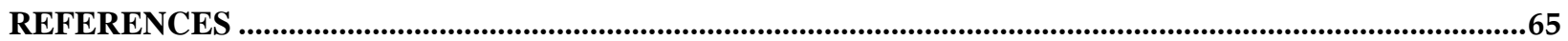

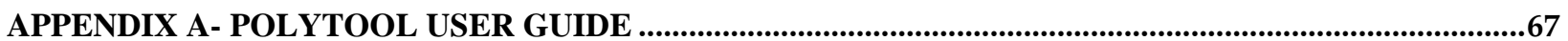

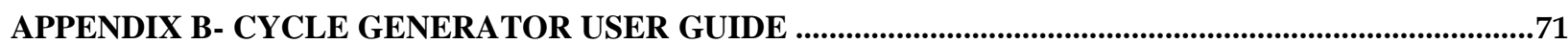

APPENDIX C- CREATED BACKBONE MODELS ....................................................................................74 


\section{List of Figures}

1.1 IBIS flowchart

3.1 PolyTool Interface

3.2 Interface for Defining Depth of Regression

3.3 Regressor Manipulation Interface

3.4 Realization of Polynomial Output

3.5 Projection of Polynomial Model

3.6 Graph Showing the Effect of the Secondary Parameter

3.7 Projection of Polynomial Model

3.8 Graph Showing the Effect of the Secondary Parameter

4.1 Genetic Algorithm Flowchart

4.2 Example of Microtrip Definition

4.3 Sample Chromosome Definition

4.4 Binary Chromosome Representation

4.5 Karyotype Alteration Example

4.6 Mutation Example (Binary Representation)

4.7 Mutation Example (Real Representation)

4.8 Crossover Example

4.9 GUI for Cycle Generator

4.10 Optimal Parameter GUI for Cycle Generator

4.11 Results Window for Cycle Generator

4.12 Results Window for Cycle Generator

4.13 Kinetic Intensity Limits

4.14 Percentage Idle Limits

4.15 Stops per Mile Limits

4.16 Standard Deviation of Speed Limits

5.1 Example Search Field

5.2 Example Search Field with Expanded Dataset

5.3 Example Polynomial Model

5.4 Example Polynomial Model with Expanded Dataset

5.5 Example Backbone Model-2006 Diesel 


\section{List of Tables}

1.1 Comparison of input parameter sets 


\section{List of Abbreviations}

CAFEE: $\quad$ Center for Alternative Fuels, Engines, and Emissions

CNG: $\quad$ Compressed Natural Gas

CO: $\quad$ Carbon Monoxide

$\mathrm{CO}_{2}$ : $\quad$ Carbon Dioxide

EMFAC: $\quad$ Emission FACtors

EPA: $\quad$ Environmental Protection Agency

g: $\quad$ grams

GA: Genetic Algorithm

gal: gallon

GUI: $\quad$ Graphical User Interface

GUIDE: GUI Development Environment

HC: $\quad$ Hydrocarbons

IBIS: $\quad$ Integrated Bus Information System

lbs: $\quad$ pounds

mph: miles per hour

MOVES: $\quad$ MOtor Vehicle Emission Simulator

NOx: $\quad$ Oxides of Nitrogen

PM: $\quad$ Particulate Matter

ULSD1: $\quad$ Ultra Low Sulfur Diesel 1

WVU: $\quad$ West Virginia University 


\section{Introduction}

In recent years, pollution has become a major societal issue. From recent Presidential elections to the "Green" movement, awareness of industrial and automotive pollution is at an alltime high. The global impact of greenhouse gas emissions is paramount to environmental contamination, whether "Global Warming" or general air-quality degradation. Therefore, testing and approximating the total effluence of new technologies is necessary. In the United States, the Environmental Protection Agency (EPA) is the overall watchdog charged with regulating exhaust emissions. While the EPA has established regulations governing the amount of certain emissions that may be produced, certain areas have created more stringent guidelines: California for example. Furthermore, transit agencies and manufacturers are becoming increasingly pressured to create "cleaner" vehicles: that is to produce vehicles that exceed federal requirements. Establishing the effectiveness of introducing new technologies into existing vehicle fleets is of note. It remains impractical to physically test each new automotive architecture, and available regulatory data for that vehicle may be inconsistent with specific "real world" usage. Therefore, an accurate and usable tool is necessary to allow fleet operators to access the effectiveness of proposed fleet configurations.

The Integrated Bus Information System (IBIS) is an online tool that allows users to predict emissions and fuel consumption for a fleet of heavy duty vehicles. Development is currently centered on transit vehicles using conventional Diesel, Compressed Natural Gas (CNG), and Diesel Hybrid Architecture.

Using the West Virginia University (WVU) Center for Alternative Fuels, Engines, and Emissions (CAFEE) database, polynomial models were constructed using specific driving characteristics. Three main categories of input parameters are considered. The first being parameters that define the architecture of the vehicle: Length, Curb Weight, Occupancy, etc. The second category defines operating characteristics, and the third environmental classifications. The modeling strategy focuses on polynomial backbone models obtained via regression using parameters from the second input category, and correction factors to address each additional input from the first and third categories. Parameters chosen to define a driving cycle were average speed with idle, standard deviation of speed with idle, kinetic intensity, percentage idle, and number of stops per mile. It was necessary to develop advanced 
computational tools to facilitate the modeling process. The development of these tools is the objective of this thesis.

The first tool developed was a polynomial regression tool; PolyTool. It was used to develop continuous models for fuel consumption and each emissions species; $\mathrm{CO}, \mathrm{CO}_{2}, \mathrm{HC}, \mathrm{PM}$, and $\mathrm{NO}_{\mathrm{x}}$. The PolyTool allowed for multiple inputs and varying degrees for the polynomial output. Additionally, the user may decide which standard driving cycles are to be used in the regression. The tool may also be modified to model different buses or fuel types given that sufficient data is available.

PolyTool is effective given that enough data are supplied, in terms of the domain of average speed. Initial testing of polynomials created with the PolyTool suggested that 12 standard driving cycles were inadequate for proper modeling of emissions and fuel consumption: this lead to the creation of an additional development tool.

The second tool created to aid in the development of backbone models was a driving cycle generator. This tool employs a Genetic Algorithm (GA) to reorder microtrips into full driving cycles. A microtrip was defined as a period of non-idle within a standard driving cycle. Considering that second-by-second data were available, these microtrips could be arranged and/or duplicated to achieve cycle characteristics not available in a full standard cycle.

The GA was selected for this tool due the capability to be formulated as an optimization problem with multiple input parameters, objectives, and constraints which do not have a tractable general analytical representation or model. The GA tool was successful in creating additional points in areas that the experimental data were lacking. This lead to a much better approximation when these data were integrated into the PolyTool. In addition to filling gaps in experimental data, probable limits for input parameters are realized with the GA tool.

Once fully integrated, the two tools allowed for a much truer approximation, due to diminishing the errors associated with interpolation and extrapolation, of emissions and fuel economy than the PolyTool was able to achieve on its own. Additionally, each tool is highly flexible to accommodate visual inspection and modification to achieve a more "real world" trend. 


\section{Chapter 1 Scope and Motivation}




\subsection{Overview of Current State of the Art Modeling of Engine Fuel Economy and Emissions}

Currently, there are several models available for predicting fuel economy and emissions; the most widely used being EMFAC, NONROAD, and MOBILE6. Additionally, there is another model, MOVES, being developed that will replace MOBILE6 and incorporate NONROAD into its output calculations.

EMFAC or the Emission FACtors model ${ }^{1}$ was developed by the California Air Resources Board with the most recent public version being EMFAC 2007. EMFAC is an "inventory model that calculates emission inventories for motor vehicles operating on roads in California." (EMFAC manual) While EMFAC can be considered a fleet model, it differs from our modeling methods in that our approach is more localized in terms of a vehicle fleet. Also, EMFAC considers non-operating pollutants such as brake and tire wear. Similar to our approach, EMFAC computes baseline emission rates derived from a unified cycle. Emissions rates for other average speeds are calculated by applying a speed correction factor. ${ }^{2}$

MOBILE6 $^{3}$ and MOVES $^{4}$ are inventory models that calculate emission output. MOBILE6 uses emission rates derived from the Federal Test Procedure cycle. Again, a speed correction factor is applied to scenarios differing from the baseline emission rate. ${ }^{2}$ As with EMFAC, the scope of this modeling is greater than our definition of a vehicle fleet. Also similar to EMFAC, these models are intended to be run in a desktop computing environment. This differs from the IBIS approach: which is to provide a user with a web based modeling environment.

In addition to the aforementioned macroscopic emissions models, there are other microscopic models of note: primarily, Virginia Tech's VT-Micro and University of California, Riverside's CMEM. CMEM or Comprehensive Modal Emissions Model is a power-demandbased emission model based on a "parameterized physical approach that breaks down the entire emission process into components that correspond to the physical events associated with vehicle operation and emission production." ${ }^{2}$ VT-Micro is a regression based model designed to be used within a microscopic simulation model. ${ }^{5}$

Each of these models differs from IBIS in that the modeling approach adopted for IBIS requires a more flexible and simplified input set of the user. This will allow a less technically trained individual to utilize IBIS to generate accurate results. For example, a driving cycle may be chosen from a set of standardized cycles or the user may opt to define the driving schedule in 
as little as two parameters. This flexibility in defining a cycle enhances the usability of IBIS; yet, comparable accuracy in the output results is maintained. Initial analysis shown below in Table 1.1, showed that second degree models for triplets of input parameters outperformed other configurations with pairs being the next most desirable. It was determined, however, that pairs of input parameters was still preferred since it would require the end user of IBIS to supply less information and would still yield an acceptable approximation.

\begin{tabular}{|c|c|c|c|c|c|c|}
\hline & Parameters & $\begin{array}{c}1 \text { st } \\
\text { Degree }\end{array}$ & $\begin{array}{l}\text { 2nd } \\
\text { Degree }\end{array}$ & $\begin{array}{c}\text { 3rd } \\
\text { Degree }\end{array}$ & $\begin{array}{c}\text { 4th } \\
\text { Degree }\end{array}$ & $\begin{array}{c}\text { 5th } \\
\text { Degree }\end{array}$ \\
\hline Average PE & \multirow{2}{*}{$\begin{array}{c}\text { Av Speed/ } \\
\text { STD }\end{array}$} & 19.946 & 7.2269 & 2.5218 & 10.006 & 44.93 \\
\hline Max PE & & 110.32 & 21.795 & 9.0462 & 23.681 & 182.85 \\
\hline Average PE & \multirow{2}{*}{$\begin{array}{l}\text { Av Speed/ } \\
\text { Stoppm }\end{array}$} & 13.052 & 10.09 & 5.0837 & 28.29 & 179 \\
\hline Max PE & & 32.685 & 22.395 & 24.368 & 96.896 & 523.82 \\
\hline Average PE & \multirow{2}{*}{$\begin{array}{c}\text { Av Speed / } \\
\mathrm{KI}\end{array}$} & 12.47 & 10.74 & 9.685 & 44.116 & 110 \\
\hline Max PE & & 40.956 & 36.005 & 33.77 & 71.826 & 589 \\
\hline Average PE & \multirow{2}{*}{$\begin{array}{c}\text { STD/ } \\
\text { Stoppm }\end{array}$} & 16.406 & 3.9439 & 1.0675 & 41.195 & 151.65 \\
\hline Max PE & & 52.831 & 13.211 & 4.3704 & 169.27 & 738.58 \\
\hline Average PE & \multirow{2}{*}{ STD/ KI } & 15.024 & 11.375 & 1.8452 & 16.902 & 172.82 \\
\hline Max PE & & 31.375 & 40.465 & 11.878 & 94.695 & 620.2 \\
\hline Average PE & \multirow{2}{*}{ Stoppm/ KI } & 15.541 & 5.168 & 3.2389 & 5.9768 & 8.8448 \\
\hline Max PE & & 45.501 & 18.009 & 14.413 & 62.979 & 110.91 \\
\hline Average PE & \multirow{2}{*}{$\begin{array}{l}\text { Av Speed/ } \\
\text { STD/ } \\
\text { Stoppm }\end{array}$} & 13.343 & 1.9688 & 82.038 & 774.29 & 1577.6 \\
\hline Max PE & & 40.546 & 4.7844 & 294.06 & 7224 & 6877 \\
\hline Average PE & \multirow{2}{*}{$\begin{array}{c}\text { Av Speed/ } \\
\text { STD/ KI }\end{array}$} & 12.493 & 5.0428 & 108.92 & 2026 & 591.81 \\
\hline Max PE & & 26.223 & 12.322 & 175.72 & 4481.2 & 591.81 \\
\hline Average PE & \multirow{2}{*}{$\begin{array}{l}\text { Av Speed/ } \\
\text { Stoppm/ KI }\end{array}$} & 13.981 & 1.209 & 56.254 & 272.42 & 1052 \\
\hline Max PE & & 38.546 & 3.3474 & 151.33 & 520.73 & 4852 \\
\hline Average PE & \multirow{2}{*}{$\begin{array}{c}\text { STD/ } \\
\text { Stoppm/ KI }\end{array}$} & 16.564 & 1.7645 & 65.661 & 184.05 & 182.59 \\
\hline Max PE & & 53.176 & 6.4693 & 287.31 & 1477.3 & 1481 \\
\hline
\end{tabular}

Table 1.1: Initial analysis comparing pairs of input parameters to triplets

Additionally, being unique to the IBIS modeling approach is the ability for the user to design a vehicle fleet of distinct configuration. That is, a user may configure multiple vehicles when defining his/her fleet. This is perhaps the most significant feature of IBIS. Whereas available software packages make assumptions about the extent of a vehicle load in a given area, IBIS allows a user to create and evaluate only those vehicles operating within his/her vehicle 
fleet. This is not to say that available methodologies are incapable of evaluating emissions in this manner, only that IBIS provides a simplified approach for developing and evaluating fleet total fuel consumption and engine out emissions.

\subsection{General Description of IBIS}

The Integrated Bus Information System, or IBIS, is an online tool designed for industry managers to support decisions regarding bus fleet procurement and operation. IBIS evaluates fuel efficiency [miles/gallon], carbon dioxide emissions [g/mile], carbon monoxide emissions [g/mile], oxides of nitrogen emissions [g/mile], total unburned hydrocarbons $[\mathrm{g} / \mathrm{mile}]$, and particulate matter $[\mathrm{g} / \mathrm{mile}]$. The user is required to provide specific information that defines one or several "vehicle types" and the total number of units for each vehicle type. These inputs are defined within three categories: Vehicle Parameters, Driving Characteristics, and External Operating Conditions.

Driving Characteristics included are as follows:

- Average Speed with Idle (This is a mandatory parameter.)

- Number of Stops per Mile

- Percentage Idle

- Standard Deviation of Speed with Idle

- Kinetic Intensity

These driving characteristics are selected due to their availability and significance in predicting emission and fuel consumption behavior determined through statistical correlation studies performed by Jun Tu. ${ }^{6}$ Additionally, the user is only required to produce a value for average speed in order to complete a simulation. In cases where additional input data are available, IBIS will compute results using pairs of parameters. Pairs are chosen over other combinations (triplets for example) since visual inspection of the models in four dimensions is impractical, and initial analysis showed that there is little benefit to expanding the models beyond pairs. More importantly, expanding beyond pairs of inputs requires the user to supply additional information regarding the driving schedule that may limit the usability of IBIS to the casual user.

Vehicle Parameters are defined as:

- Fuel Types

- Drive Type

- Length 
- Model Year

- Curb Weight

- Occupancy

- Rated Power

- Aftertreatment

- Displacement

- Number of Cylinders

- Transmission Type

- Type of Heating System

- Capacity of Cooler

External Operating Conditions are:

- Road Grade

- Geographical Location

- Season

IBIS is currently under development with an emphasis in creating backbone models for two major fuel types (Ultra Low Sulfur Diesel 1 (ULSD1) and Natural Gas (CNG)), and different vehicle model years. Additionally multiplicative deltas are being developed using the road-load equation and computed ratios from the WVU CAFEE testing database.

Generally, IBIS is comprised of backbone models created through regression analysis and additive and multiplicative correction factors. The backbone models are generated through the combined use of the driving cycle generator and the PolyTool. Using existing test data, the available driving cycles are expanded to account for areas unrepresented in the regression database, and regression is preformed on the expanded set. Additionally, correction factors developed through the adaptation of the road-load equation, analysis of experimental data, and other studies are applied to the backbone models to account for a variety of effects.

\subsection{Analysis of Computational Needs, Requirements, Available, and Developed Methodologies}


IBIS is a tool born from extensive chassis dynamometer testing by WVU CAFEE. This tool is a compilation of both backbone models and correction factors to account for varying vehicle architectures and engine technologies. Development of IBIS began with a data extraction and processing effort to obtain a set of accurate testing data. This data set was used in the creation of backbone models. The polynomial tool mention herein, was the first tool to be developed. This tool relies on multiple variable polynomial regression to construct backbone models. The tool was developed initially using a 2000 model year diesel transit bus, but has since been expanded using diesel hybrid, CNG, and newer model year diesel buses.

For the creation of each backbone, additional data was needed to ensure that each model was accurate for the entire domain of inputs. The solution to this was a second tool developed that used a genetic algorithm. This tool generated additional virtual driving cycles using secondby-second emissions and fuel economy data that were inferred from experimental data and concatenated into complete driving schedules. This data were then reintroduced, including the newly created cycles, to the polynomial regression tool. Using the newly expanded database, more accurate models were created for IBIS.

Following model creation, repair algorithms were applied to each model. These repairs limit the model function in a way that non-real or improbable values were filtered. Inaccuracies may be exhibited due to the proximity of the model to zero in certain regions of the domain, or by measurement errors present within the second-by-second data. In addition to repair functions, correction factors were created to be applied to the backbone model. This method was used for a variety of reasons. Most importantly being that backbone modeling was time consuming and it was unreasonable to assume that there was sufficient data available to cover all possible vehicle configurations and technologies. Specifically, older model years (prior to 1990) have not been tested as thoroughly often only having been tested on a single driving schedule. In this case, expansion of the dataset would yield few additional cycles, and regression would fail in producing a reliable model. Also, in cases where certain aftertreatment technologies are appliednamely particulate filters- correction factors can be reliably applied eliminating the need to create a separate backbone model.

Once all models and repair/correction algorithms were established, they were introduced to an offline version of IBIS. This version was created using MATLAB, and was mostly a tool used for development purposes and evaluation of program logic. From this model, IBIS was 
converted to a web based tool programmed in PHP. Figure 1.1 shows this general modeling strategy for IBIS. 


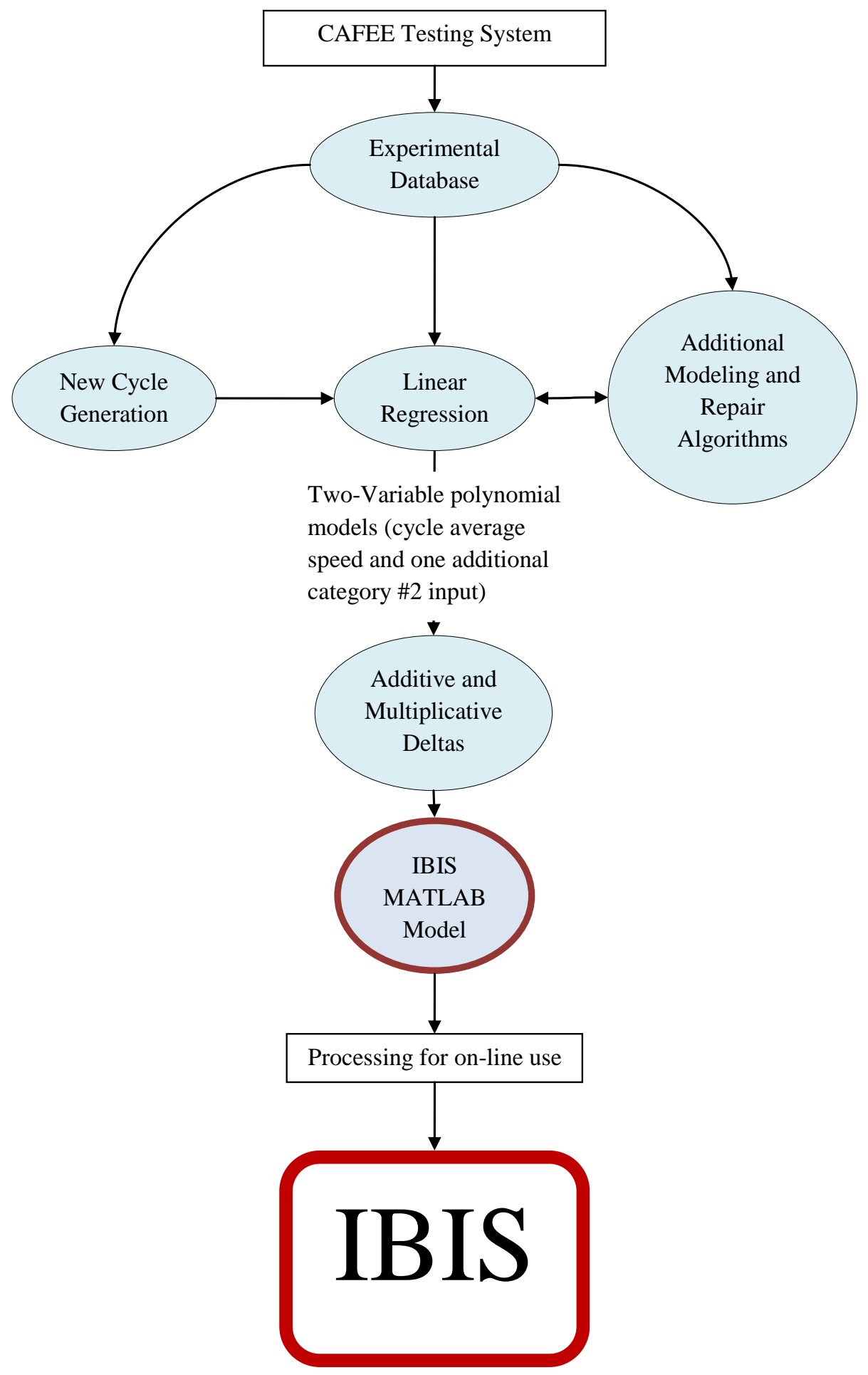

Figure 1.1: General modeling strategy for IBIS 


\section{Chapter 2 Methodology}




\subsection{General Description of The Interactive Computational Environment for Modeling Engine Fuel Economy and Emissions}

Each tool created to aid in the development of IBIS must meet certain expectations. First and foremost, since IBIS will be an ongoing project, each tool used in its development must be flexible and adaptable to accommodate changing vehicle technologies. Secondly, since IBIS will be maintained by faculty and students of WVU, the staff responsible for developing future models will be continually evolving. Therefore, each tool should be user friendly, portable, and the background code of each tool should be easily manipulated.

The goal of the polynomial tool was to perform multiple variable regression analysis on a given set of input data. This data were taken from the WVU CAFEE database. Once the data were processed into the correct data structure for the tool, regression was preformed. However, this regression should be flexible, in that, a user can define which driving cycle parameters he/she desires to include in the regression. Currently, five driving parameters are used, but the tool should allow for expansion beyond these current parameters. Secondly, the tool should allow the user to specify the regressor and to what degree the polynomial will be created.

The user will be presented with a list of available driving cycles, for which experimental information is available. The user may then select which cycles he/she would like to be included in the regression. Finally, the user will be presented with a set of output plots by which to evaluate the polynomial that is generated. Also the user should be able to manipulate terms in the regression algorithm and reevaluate the polynomial based on the changes. This step is necessary since there is a certain amount of intuitiveness in evaluating the fit of the polynomial since it must align with "real world" expectations. Also, the tool should allow for assessing the interpolative and extrapolative capabilities of the model.

The goal of the cycle generator is to construct additional driving cycles based on available experimental second-by-second data. This tool, while largely reliant on target traces of a driving cycle, requires flexibility to adaptation as well. The tool should be capable of importing new microtrip databases given that new testing data may not be represented by the same cycles in all cases. The tool should allow a user to specify the target driving parameters, and indicate each parameter's significance to the final driving cycle. The user should also be allowed to modify the initial conditions of the tool. This will include the size of the initial population, the number of generations (iterations), the number of individual in the initial 
population, and the maximum relative error for each input parameter. These initial conditions are relevant to the operation of the GA, and generally will only affect the output cycle in terms of the extent of calculations preformed by the tool.

Following input and execution of the tool, the user should be presented with a summary of the cycle created, and given the option to continue for an additional amount of generations. The user will be presented with a graphical realization of the cycle as well as the driving cycle parameters achieved in the final cycle.

\subsection{Polynomial Modeling Based on Linear Regression- Theoretical Background}

Statistically speaking, regression analysis is simply a modeling/analysis method for evaluating a dataset of dependent variables and one or more independent variables. Regression is effective in modeling cause/effect relationships, prediction, and inference. The most common form of this analysis is the Least Squares Method.

The term linear regression refers to linearity in terms of the model output, not with respect to the independent variables. That is, "linear regression problems can have modeling functions that are linear or nonlinear functions of independent variables." "7 The least square method is usually assumed initially since modeling parameters are assumed to be unknown constants. The general form for the least squares method is as follows:

$$
z=X \theta+v
$$

\section{Where}

$z$ is a vector of measurements

$\theta$ is a vector of unknown parameters

$v$ is a vector of measurement errors

$X$ is a matrix of vectors of ones and regressor

For the least squares method, $v$ is assumed to be zero mean and uncorrelated, with constant variance. ${ }^{7}$ The best estimator of $\Theta$ comes by minimizing the sum of the squared differences between measurements of the model.

More specifically, for the polynomial tool being developed, the parameter estimation process requires the following:

- A model structure with unknown parameters $\theta$ to be estimated.

- Measurements z. 
- A mathematical model for the measurement process.

- Assumptions about the uncertainty in the model parameters $\theta$ and the measurement noise $v$.

The least-squares approximation model was employed to estimate the model output. For the least-squares method, $\theta$ is a vector of unknown constant parameters (this is to be determined), and $v$ is a random vector of measured noise.

The model is defined by a polynomial $\mathrm{PN}(\mathrm{x})$ of a pre-specified degree $\mathrm{N}$.

Let $\mathrm{x}=\left[\mathrm{x}_{1}, \mathrm{x}_{2}, \mathrm{x}_{3}, \ldots, \mathrm{x}_{\mathrm{n}}\right]^{\mathrm{T}}$ represent the model parameters (average speed, kinetic intensity, number of stops per mile, standard deviation, and/or percentage idle).

Then:

$$
P_{n}(x)=\sum_{j=0}^{\bar{N}} \theta_{j} \prod_{i=1}^{m} x_{i}^{b_{i j}}
$$

where

$$
\bar{N}=n+\frac{n=1}{2} \sum_{k=1}^{N-1} n^{k}
$$

Eq. 2.2

For example, for $\mathrm{N}=2$ and $\mathrm{n}=3$. The model is a second degree polynomial with three parameters considered.

$$
\bar{N}=3+\frac{3+1}{2} \sum_{k=1}^{1} 3^{k}=3+2 \times 3=9
$$

Eq. 2.3

This tells us that the polynomial will have 10 terms and that there are 10 unknowns to be determined.

$$
\theta_{j} \text { where } j=0,1,2, \ldots, 9
$$

therefore 


$$
P_{2}(x)=\sum_{j=0}^{9} \theta_{j} \prod_{i=1}^{3} x_{i}^{b_{i j}}=\sum_{j=0}^{9}\left(\theta_{j} x_{1}^{b_{1 j}} x_{2 i}{ }^{b_{2 j}} x_{3}{ }^{b_{8 j}}\right)
$$

This model is called linear in the parameters $(\theta)$ if the output $\mathrm{y}$ is given by the following expression:

$$
\begin{gathered}
y=H \theta \text { where } H \text { is known } \\
\theta=\left[\theta_{0} \theta_{1, \ldots,} \theta_{\hat{N}}\right] \\
H=\left[\begin{array}{cccc}
1 & h_{11} & \ldots & h_{1 \widehat{N}} \\
1 & h_{21} & \ldots & h_{2 \widehat{N}} \\
\vdots & & & \vdots \\
1 & h_{\hat{N} 1} & \ldots & h_{\hat{N N}}
\end{array}\right]
\end{gathered}
$$

where $h_{i j}$ is the value of the regressor jor the measurement set $i$.

In the previous example, regressor $j=5$ is $x_{1} \times x_{3}$.

If $\mathrm{z}$ is a vector of measurement of the output, for example fuel economy, then:

$$
z=H \theta+v
$$

where $v$ is measurement error, which is assumed to be zero and uncorrelated, with constant variance:

$$
\begin{gathered}
E(v)=0 \\
E(v v)^{T}=\sigma^{2} I
\end{gathered}
$$

The least-squares model, given the measurement $\mathrm{z}$, the "optimum" estimate of $\theta(\hat{\theta})$ is obtained by minimizing the weighted number of squared differences between the measured outputs and the model outptuts.

$$
y(\theta)=\frac{1}{2}(z-H \theta)^{T} R^{-1}(z-H \theta)
$$

where $R^{-1}$ is positive definite 
The estimate $\hat{\theta}$ of $\theta$ that minimizes $y(\theta)$ must satisfy:

$$
\begin{gathered}
\frac{\partial y}{\partial \theta}=-H^{T} z+H^{T} H \hat{\theta}=0 \\
=>H^{T} H \widehat{\theta}=H^{T} z \\
\Rightarrow>H^{T}(z-H \widehat{\theta})=0 \\
\therefore \quad \hat{\theta}=\left(H^{T} H\right)^{-1} H^{T} z
\end{gathered}
$$

The regressor must be linearly independent, then $\left(H^{T} H\right)^{-1}$ exists

The covariance matrix of the estimate $\hat{\theta}$ or covariane matrix of estimation error $\hat{\theta}-\theta$ is:

$$
\operatorname{cov}(\tilde{\theta})=\left(H^{T} H\right)^{-1} H^{T} E\left(v v^{T}\right) H\left(H^{T} H\right)^{-1} \cong \sigma^{2}\left(H^{T} H\right)^{-1}
$$

The correlation matrix of $\hat{\theta}$ shows if any regressors are inlearly dependent

$$
\begin{gathered}
\operatorname{cov}\left(\hat{\theta}_{j}, \hat{\theta}_{K}\right)=\sigma^{2} d_{I K} \text { where }\left[d_{j k}\right]=\left(H^{T} H\right) \\
r_{j k}=\frac{d_{j k}}{\sqrt{d_{j j} d_{k k}}} \\
\operatorname{corr}(\hat{\theta})=\left[r_{j k}\right]
\end{gathered}
$$

Eq. 2.10

\subsection{Genetic Algorithm Optimization- Theoretical Background}

The development of continuous models using polynomial regression is dependent on a large number of data points. During construction of models for the IBIS tool, this problem was encountered. The selected reference bus was represented by only 12 standard driving cycles. This bus is determined to be indicative of its model year group and closely exhibits behavior consistent with vehicles sharing similar architectures; therefore, it is chosen to be modeled as a backbone model. It was necessary to expand this selection to represent a greater coverage of the range for average speeds and other parameters that characterize the driving cycle. A cycle generation tool was constructed to address this issue. 
A genetic search method was the chosen approach for this tool. The Genetic Algorithm (GA) is an iterative search technique inspired by Darwin's theory of Natural Selection. Charles Darwin introduced this theory in 1859 , and it has since become a cornerstone of modern biology ${ }^{8-9}$ The theory states that certain favorable traits become more common in successive generations whereas less favorable traits become increasingly less common. This occurs largely due to the ability of organisms with the favorable traits to survive to produce future generations. That is, the organism begins to adapt to changes in its ecosystem. An example of Darwin's theory is the antibiotic resistance developed by Staphylococcus Aureus, the common pathogen found in nearly all hospitals in the world. ${ }^{10}$ The preferred treatment of S. Aureus infection is penicillin, but certain strains have evolved to become resistant to this treatment. In fact it has been estimated that by $196080 \%$ of hospital isolates had become penicillin resistant. ${ }^{11}$

The GA takes inspiration from biological processes, in that they attempt to simulate the progression of favorable traits from one generation to another. The GA was first theorized in the late 1950 's. ${ }^{8-9}$ At first the GA was simply intended to model natural evolution. It was not until later that the GA was employed to function as a tool for machine learning and optimization. The first book published to systematically outline the mimicry of biological revolution as a problem solving strategy was J. H. Holland's Adaptation in Natural and Artificial Systems: An Introductory Analysis with Applications to Biology, Control, and Artificial Intelligence published in $1975 .{ }^{12}$

The GA is generally employed for optimization. Opposed to more widely used enumerative and gradient techniques, GAs are capable of covering a broader spectrum of problems. A gradient method asserts that in an infinite or nearly infinite search space the extremum of a function can be proved to exist where the derivative of a function is canceled. This gradient method, however, must also satisfy additional conditions, and exhibits the following drawbacks:

- There must be a well defined model of the optimization problem (an analytical function that will be optimized) and the model must comply with certain conditions (e.g. continuity)

- The initial conditions must be selected such that they exist in the vicinity of an extremum

- There is generally uncertainty concerning the local or global nature of the optimization to be determined

- The optimization approach may tend to focus on local maxima 
- Gradient methods are often sensitive to numerical accuracy due to the numerical computation of derivatives

The above stated problems can be largely, if not completely, avoided with the use of a genetic algorithm. GAs are particularly efficient once the solutions of the problem can be encoded to chromosomes in the GA. GAs are most powerful when the search space is large and multidimensional, a limited (or lack thereof) mathematical model exists, and when more traditional optimization methods fail, also multiple criteria and constraints can be handled without the need to incorporate them in a consistent analytical framework.

This is not to say the GA is without its own drawbacks. While the GA is simple and robust, the GA can fail when a limited number of iterations are performed, i.e. the optimization is yet to reach the global maximum. A GA can take days to solve a problem of medium complexity. Therefore, computational power is also of note. Although with the technological improvement of computer processors and other hardware design, computational power is becoming of lesser concern when considering the GA. Also, since the design of GAs employ random modifications, fatal errors (that are often difficult to predict and debug) can occur.

GAs are also better equipped to handle problems for which a "brute-force" approach would prove too computationally exhausting. The GA exhibits additional advantages over conventional search and optimization techniques. Firstly, Genetic Algorithms operate on a parallel basis. Where most iterative search techniques can explore solutions in one direction only, and are generally dependent on initial conditions leading to local maxima, the GA can search multiple areas at once. This allows for more maneuverability within the search field. If one path leads to a dead end, it can be eliminated without need to abandon all previous work and start anew.

GAs also benefit in that the performance criterion do not have to be expressed as a function. Therefore, there are no requirements for derivability, continuity, or bijectivity: the criteria may be chosen without heuristic or analytical techniques.

Given enough iterations, the GA will not be trapped into local maxima, and are consequently capable of handling data in a very large, multidimensional search space. This is accomplished by marrying the concept of altering existing solutions, and looking to new solutions to meet our specific needs. This is a result of the GA's independence of the problem solution. 
A classic example problem where GAs prove effective is the traveling salesman problem. This example can be applied to various "real world" problems involving path optimization. The traveling salesman problem states that one is to find the optimal path for a salesman to travel from city to city given a number of cities and the respective traveling cost to move between each city. More specifically, one must find the cheapest round-trip cost for the salesman. Where the number of cities is small it is possible to solve the problem with a brute force approach, testing each combination on cities to find an answer. However, should the number of cities become large the solution space is represented by $1 / 2(n-1)$ ! where $n$ is greater than 2 and $n$ is the number of cities visited. For example when $n=4$ the search space is 3 and when $n=10$ the search space becomes 181,440 . It is realistic to assume that the number of cities would be larger than 10 in a real-world simulation. This leads to a computationally expensive, iterative approach to the solution of the problem. For the cycle generator, the brute force method is exceptionally infeasible since the search space is practically infinite.

Genetic Algorithms are an ideal approach to solving this problem. The GA operates by randomly evaluating points across the whole of the search space. Each subsequent iteration focuses its evaluation around these points. The points with the greatest performance will receive a greater chance to contribute to the next generation. This addresses the caveat in natural selection; good parents can produce bad offspring, and bad parents can produce good offspring.

While the traveling salesman problem is a classical example of the use of GAs for path optimization, GAs are not limited to such applications. For example, GAs have been used in design of journal bearings ${ }^{13}$ and hydrogen storage tanks. ${ }^{14}$ Additionally, GAs have been used to design composite plastics ${ }^{15}$, heat exchangers ${ }^{16}$, and even fingerprint matching. ${ }^{17}$ Furthermore, GAs may be used in designing control systems. Control design using GAs has been employed for developing a biped locomotion controller ${ }^{18}$, a controller for an autonomous helicopter $\operatorname{model}^{19}$, and various complex nonlinear parameter optimization problems in flight controls. ${ }^{20-23}$ GA optimization and design applications are virtually limitless. 


\section{Chapter 3 \\ Interactive Computational Tool for Polynomial Modeling of Engine Fuel Economy and Emissions Based on Linear Regression}




\subsection{Algorithm development}

The PolyTool was developed such that the models produced can be easily examined and modified. The complex nature of emission modeling is such that traditional methods of determining the "fit-ness" of a polynomial to the original data are unrealistic. There is much intuitiveness involved in examining the trend of emissions as a result of increasing a driving paramters in multiple dimensions. Additionally, due to the lack of very large amounts of data, interpolation within the models can produce ill effects.

The tool was developed to address these concerns. The algorithm is capable of handling a limitless number of input parameters, but the implemention involves only the five driving parameters chosen to represent real-world driving. Average speed [mph] is paramount to describing the trend of both the emissions and fuel consumption. Therefore, average speed (with idle), calculated as total mileage divided by total time including idling periods, is chosen as the primary input of the PolyTool, and must be used in the regression process. Consequently, each of the other input parameters are referred to as secondary parameters. These parameters are defined as follows:

Stops per mile: total number of idle periods within a increment of distance including scheduled and traffic stops.

Standard Deviation of Speed: the variability of speed from the mean within a driving cycle

Percentage Idle: total time of idling periods divided by the total cycle time

Kinetic Intensity: the ratio of characteristic acceleration to the square of aerodynamic speed. This value is calculated from the driving cycle. Kinetic intensity equations are created by adapting the road-load equation. ${ }^{6}$

The algorithm also allows the user to specify which standard driving cycle, for which experimental data is available, will be used in the regression.

Upon initialization of the tool the user must set the degree of the polynomial and specify the ouptut (fuel consumption, $\mathrm{CO}, \mathrm{CO}_{2}, \mathrm{NOx}, \mathrm{PM}$, or $\mathrm{HC}$ ). Then the user choses one or more secondary parameters. Following this the user defines which cycles are to be used.

Once all user defined inputs are completed, the algorithm ${ }^{8,24}$ loads the corresponding data for the selected output parameter. Next, a b-matrix (a matrix comprised of the exponential terms of the regressors) is created in relation to the specified number of inputs and degree of the 
polynomial. For example, the user wants a $4^{\text {th }}$ degree polynimial $(n=4)$ using average speed and percentage idle ( 2 inputs $\mathrm{N}=2$ ). A matrix is created with the following structure:

$\left[\begin{array}{cc}0 & 1 \\ 0 & 2 \\ 0 & \vdots \\ 0 & n \\ 1 & 1 \\ \vdots & \vdots \\ n & n\end{array}\right]$

Next the matrix is modified such that any row who's sum is greater than $\mathrm{n}$ is removed. For example:

$$
\left[\begin{array}{ll}
2 & 3
\end{array}\right] 2+3=5>n:\left[\begin{array}{ll}
\frac{2}{7} & 3
\end{array}\right]
$$

Eq. 3.2

Finally a free term $[00]$ is added to the top of the matrix and a modified identity matrix $I_{N}(n)$ is appended to the bottom of the matrix resulting in the final b-matrix shown below.

$$
\left[\begin{array}{cc}
0 & 0 \\
0 & 1 \\
0 & 2 \\
0 & \vdots \\
0 & n \\
1 & 1 \\
\vdots & \vdots \\
n & n \\
4 & 0 \\
0 & 4
\end{array}\right]
$$

Eq. 3.3

The b-matrix represents the value of the exponent for each term in the polynomial.

$$
\theta_{0}{ }^{b_{11}} \theta_{0}^{b_{11}}+\theta_{1}^{b_{21}} \theta_{1}^{b_{2 n}} \cdots \theta_{m}^{b_{m 1}} \theta_{m}^{b_{m n}}=\theta_{0}{ }^{0} \theta_{0}{ }^{0}+\theta_{1}{ }^{0} \theta_{1}{ }^{1} \cdots \theta_{m}{ }^{0} \theta_{m}{ }^{4}
$$

where $m$ is the length of column 1 in the b-matrix

Eq. 3.4 
Following the creation of the b-matrix, the H-matrix is composed of the values of the regressors. Assuming $\mathrm{Hp}_{0}$ to equal 1:

$$
H_{m, j}=\left(X_{m, j j}^{b_{j i j j}}\right) H_{p_{0}} \text { then let } H_{p_{0}}=H_{m, j}
$$

where $m=[1$, number of rows in input data], $j=[1$, number of rows in $b$ martix], and $j=[1$, number of columns in $b$ matrix

Next let:

$$
H H=H^{s} H
$$

then

$$
\theta=\left(H H^{-1} H^{\prime}\right) z
$$

where $z$ is a vector of emission/fuel consumption values being modeled with the polynomial

Finally, the output polynomial is of the form:

$$
y=H \theta
$$

Eq. 3.7

\subsection{Implementation}

The Interactive Tool for Polynomial Modeling of Engine Fuel Economy and Emissions (PolyTool) was developed to provide a continuous model for a specific bus operating on standard driving schedules. Data were provided from the WVU CAFEE database. For development, the reference bus was a 2000 Orion bus operating on Ultra Low Sulfur Diesel fuel with a testing weight of 33,300 lbs. The reference bus uses a Detroit Diesel engine and is without aftertreatment.

The primary focus of the PolyTool was to develop models while maintaining usability. Therefore, an interface is developed using the GUI Design Environment (GUIDE) feature of MATLAB. GUIDE allows user development of GUIs in an interactive, drag and drop, manner. 
The initial interface will allow the user to define parameters for the regression process. Figure 3.1 shows the primary interface window. In this window the user can specify the output that will be evaluated. Additionally, this is where the inputs, degree of polynomial, and parameters, are defined. The tool is capable of using several parameters, but through testing, and for usability and simplicity in IBIS pairs of input parameters were deemed adequate. Based on correlation studies five input parameters were selected for implementation in this version of the PolyTool. However, Average Speed is necessary for a good fit since it can be correlated to each of the other parameters. ${ }^{5}$ Additionally, while there are several parameters that can define a driving cycle, only a select few are implemented at this time.

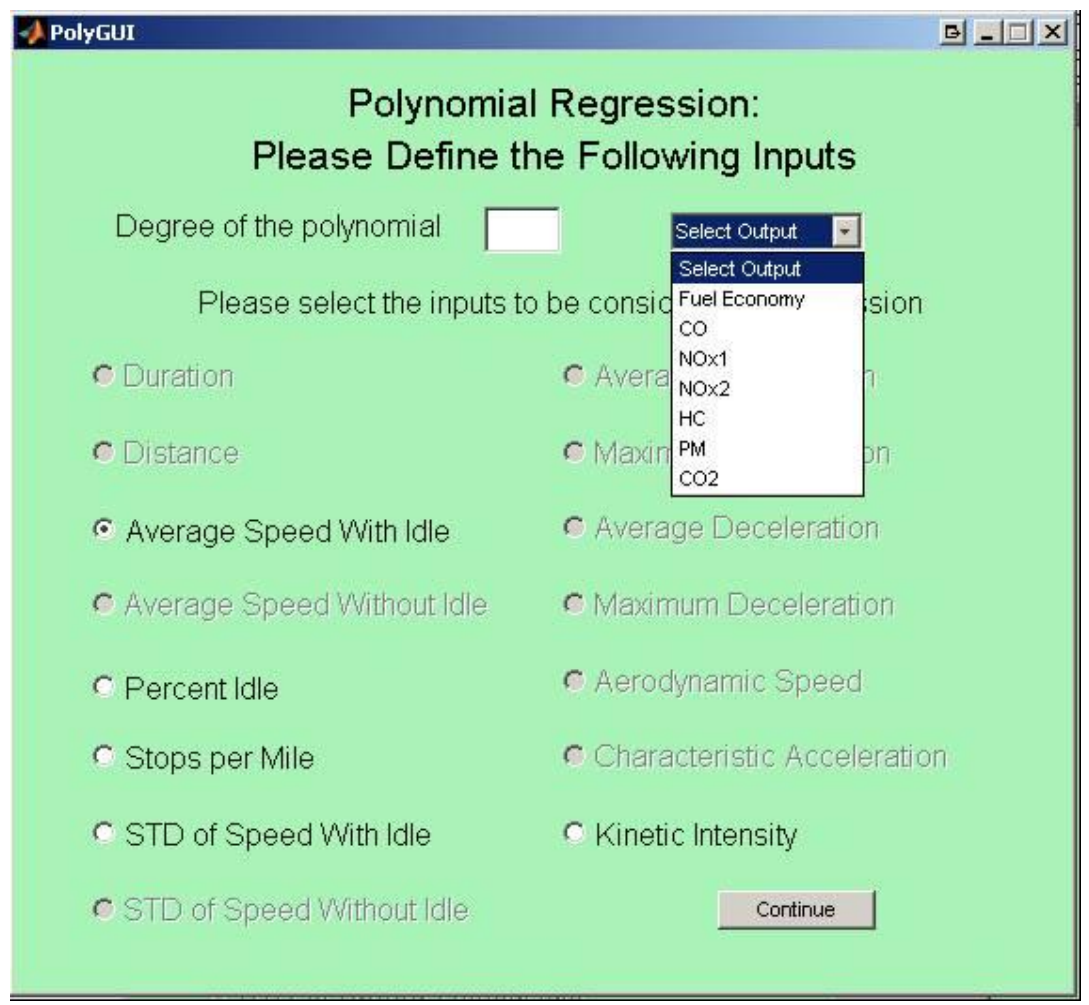

Figure 3.1: Interface where a user specifies input parameters

The following interface is where the user defines which standard cycles will be used for evaluation. Figure 3.2 shows this interface. 


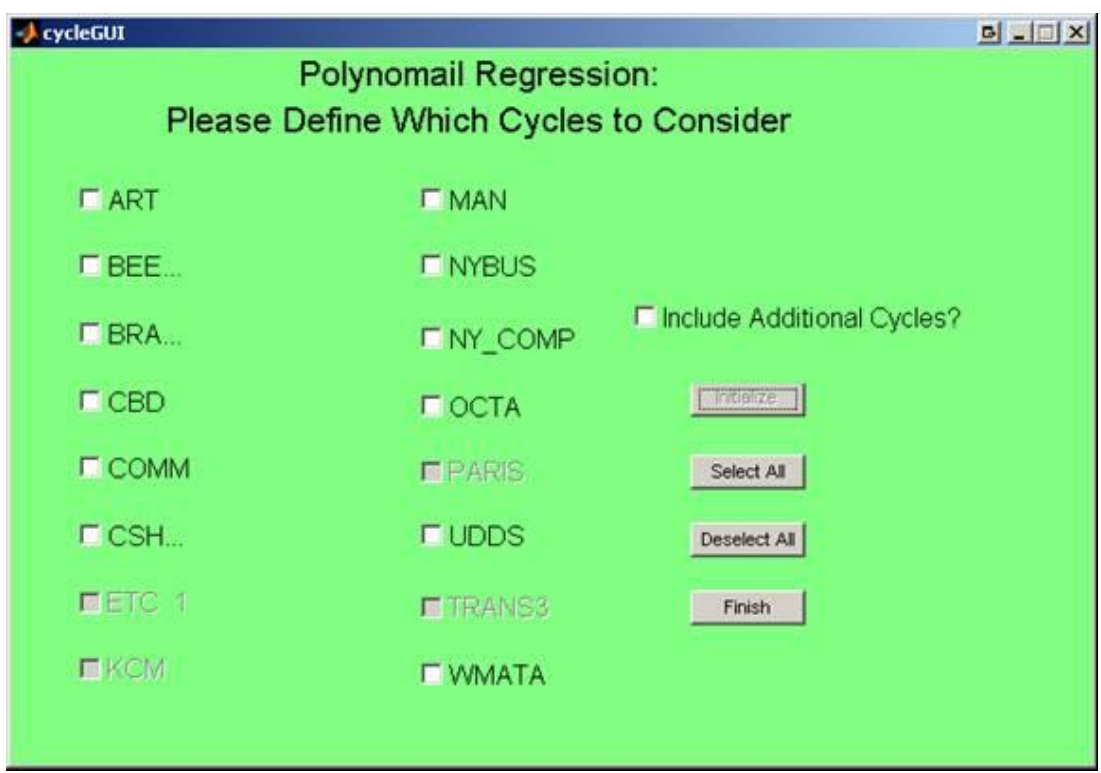

Figure 3.2: Interface to define depth of regression

For the case of the bus used for development, although 16 cycles are represented in the database, 4 cycles were either atypical or considered to be outliers and were consequently omitted from this version of the tool.

Upon running the tool, several output windows are shown. Among these are graphical realizations of the polynomial in a two dimensional space and a menu that allows the user to manipulate the solution by deleting terms from the regression, namely terms that appear not to be relevant. Figure 3.3 shows this option for removing terms from the list of regressors.

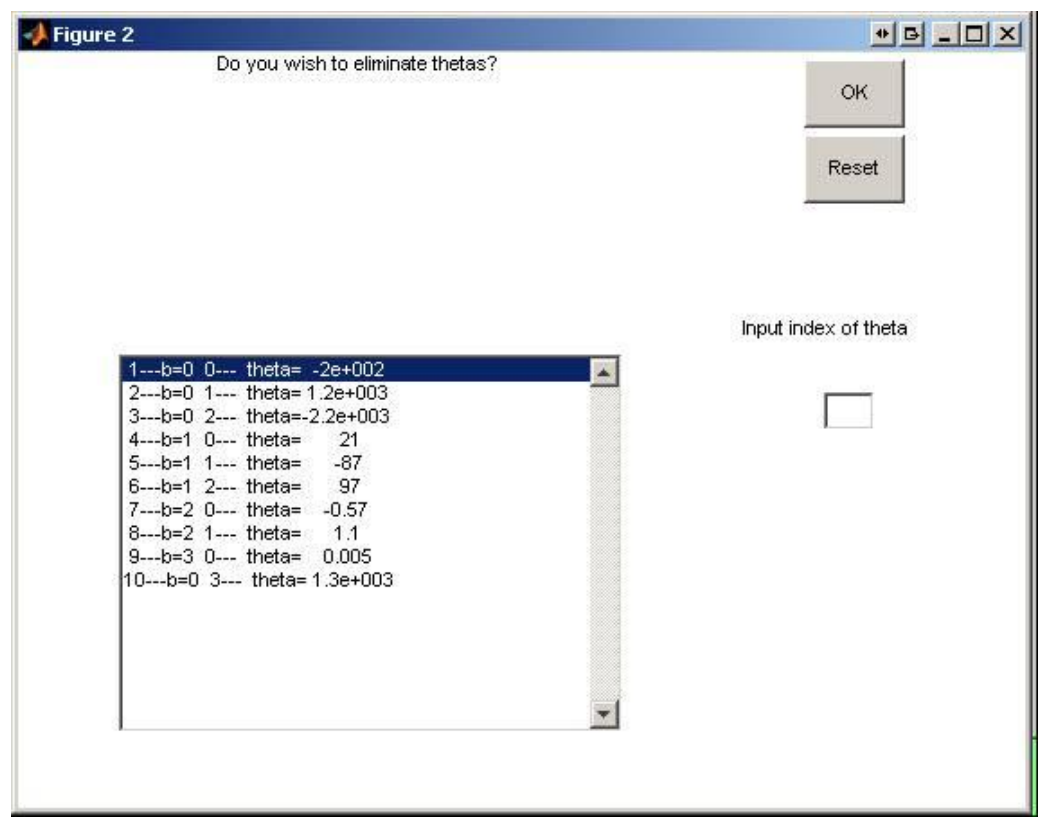


Figure 3.3: Menu allowing users to manipulate terms in the regression

The graphs supplied for evaluation by the tool are discussed in the following section as well as in the results chapter, Chapter 6.

\subsection{Backbone Model Development Using the Polynomial Regression Tool}

In this section, an example is illustrated to show the method for developing models for fuel economy and emissions using the regression tool outlined herein. This example does not reflect an actual model to be used in IBIS, but is merely presented as an illustration to describe the use of the developed tool. Examples of models developed for IBIS can be found in appendix C of this text.

The user begins by running and initializing the program. At this point the user defines the degree of the target polynomial, the desired output, and input parameters. These selections are made in the window as shown in Figure 3.1. For this illustration, a polynomial of degree 5 is selected to predict fuel economy using average speed with idle and number of stops per mile.

Next the user is presented with the window shown in Figure 3.2. For this example, all driving cycles were selected as well as a previously created database of additional cycles produced using the GA-based approach. The bus being modeled, in this case, was a 2006 diesel bus.

Upon a successful run, several results windows are presented. The number of graphs displayed varies dependent upon the total number of cycles included in the regression, in this example close to 300. Of these windows, included is a realization of the model output with respect to each parameter, such as those shown in Figures 3.4. 

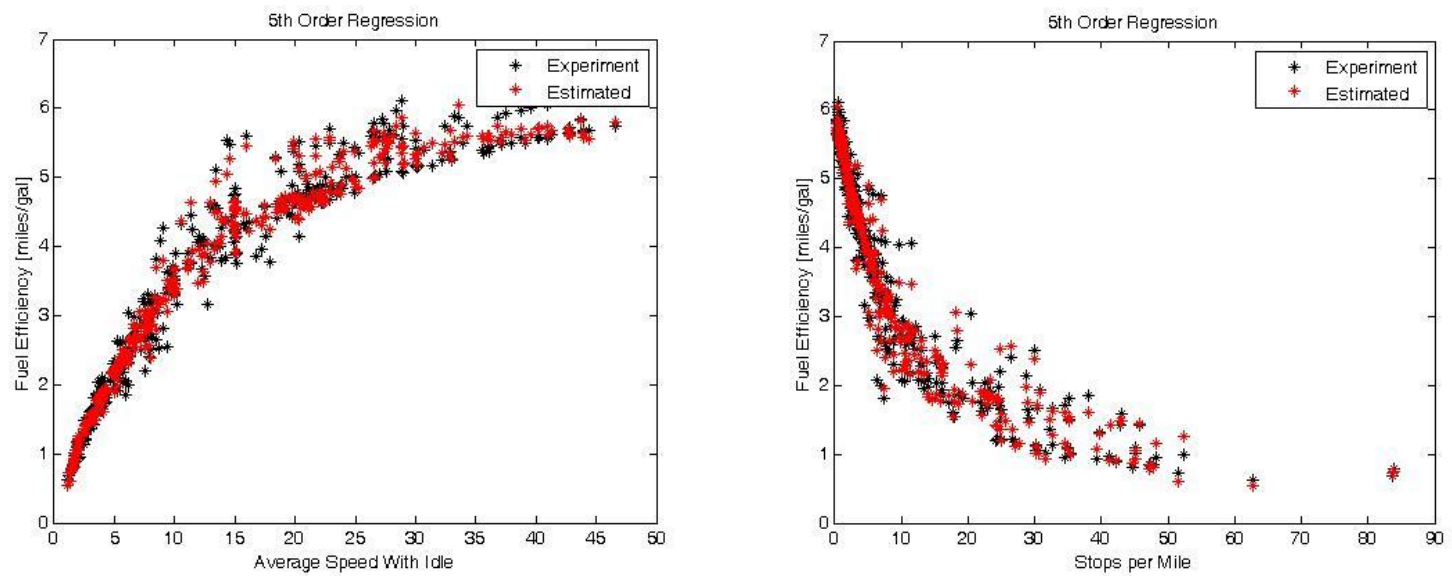

Figure 3.4: Graphical realizations of polynomial output

This realization is useful in seeing the general trend and fit of the polynomial, but the following Figures illustrate better the effect of the secondary parameter on the overall fit of the model.

Figure 3.5 shows a continuous graph of the model displayed as a 2 dimensional projection. While it may seem as if the model has discontinuities, these sharp edges are a result of the secondary parameter's effect on the output. At each point on the graph the second parameter is varying.

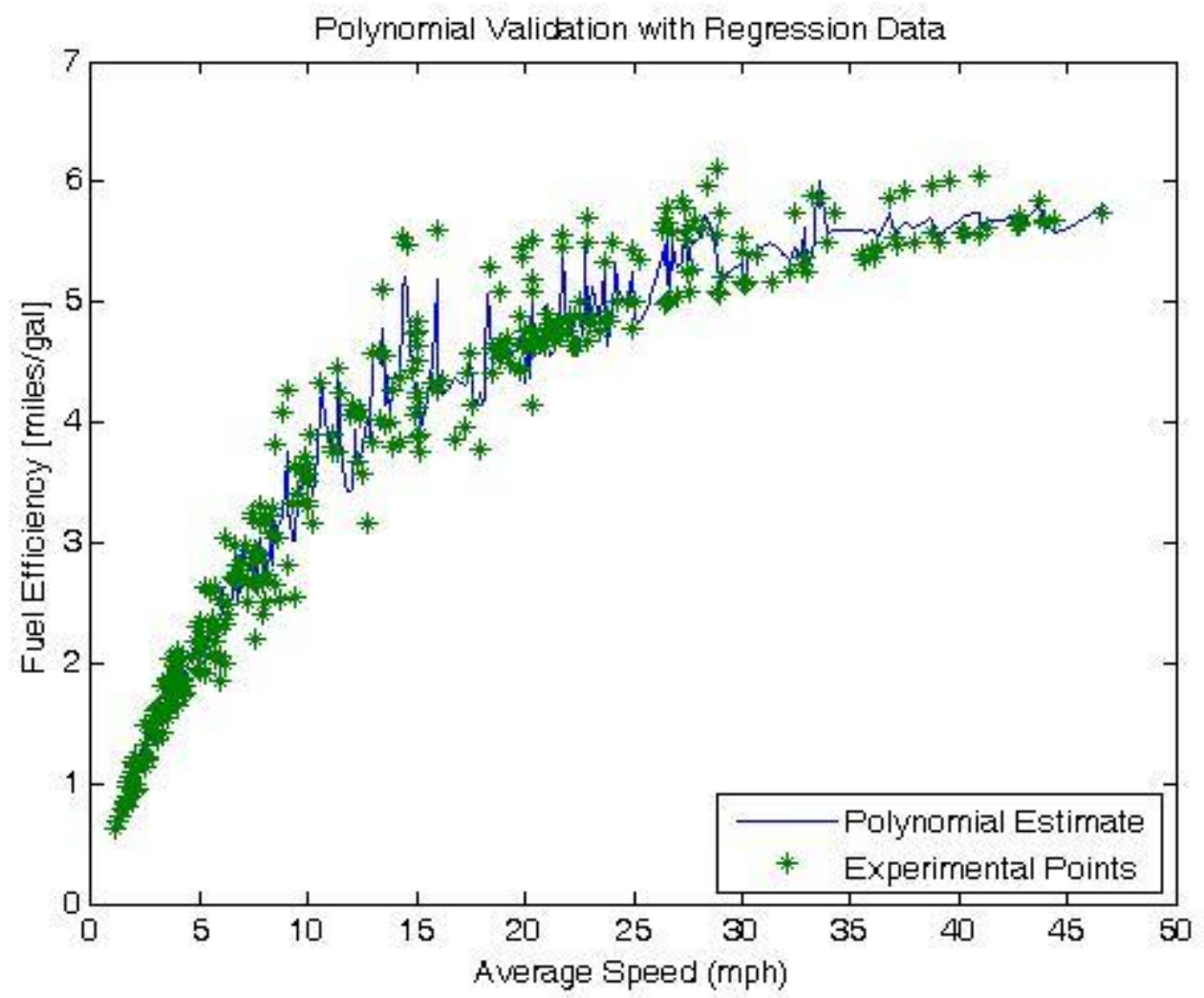


Figure 3.5: Projection of the model using average speed and stops per mile

As evident in the previous Figure, the secondary parameter influences the trend of the polynomial and must be evaluated. The next set of Figures show additional output windows of the tool. These windows, shown in Figure 3.6, illustrate the effect of making the secondary parameter constant and varying the average speed only. Regression points with a secondary parameter within $20 \%$ of the fixed value are shown in magenta and the fixed value is shown in red. Using these windows allows the user to evaluate the fitness of the model when considering the magenta points.

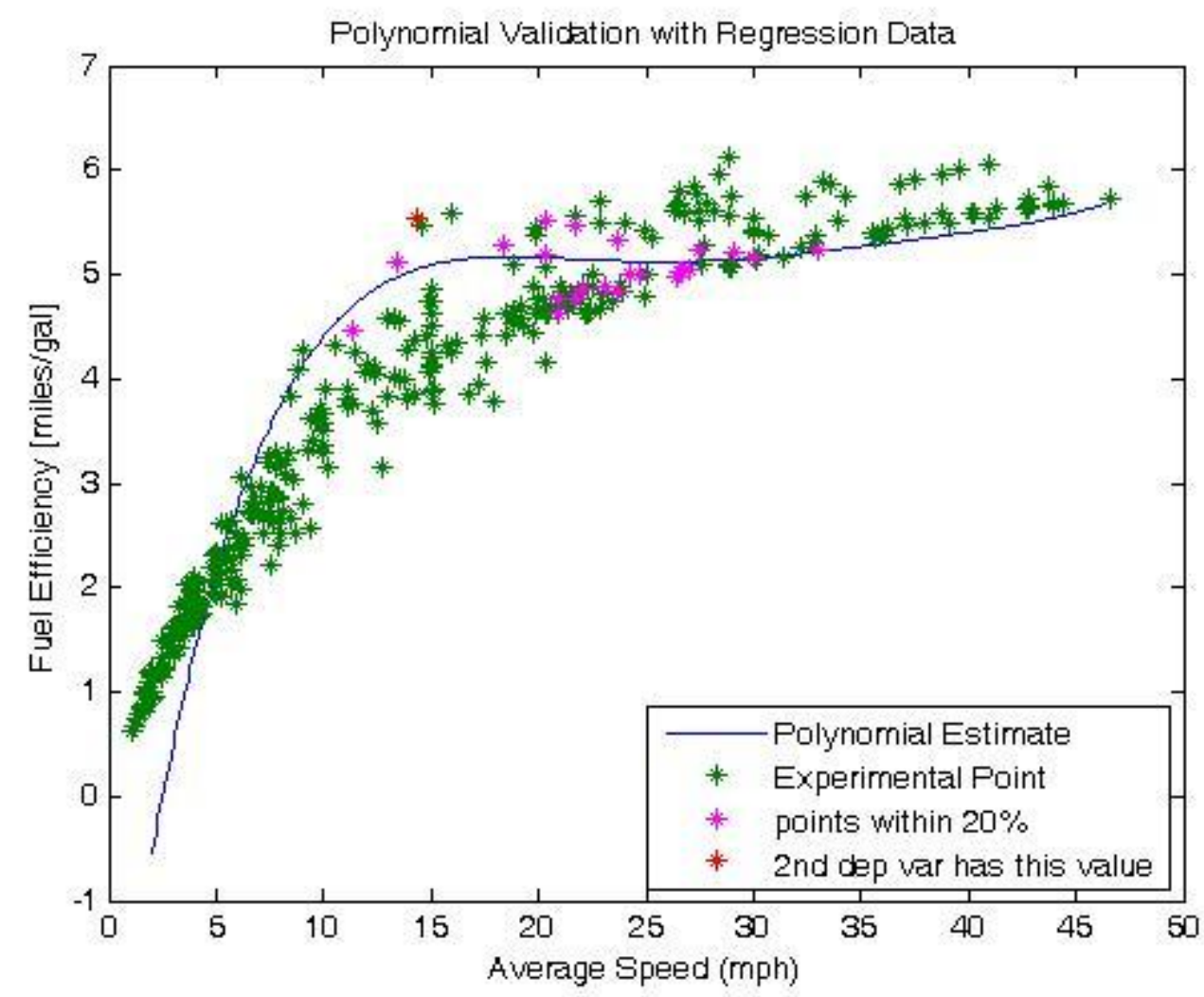

Figure 3.6: Model plotted using a fixed value for the secondary parameter

Upon inspection of Figure 3.6, we can see that there is a decent fit for those points having a value within $20 \%$ of the fixed value. However, this fit may be improved. It is determined that the secondary parameters play a diminishing role in emissions prediction since each parameter can be correlated to average speed. Additionally average speed is assumed to be a limiting factor for the magnitude of each secondary parameter. For example it is improbable to assume a cycle to have a large average speed and a large number of stops per mile. Consequently, it is necessary 
to alter the model such that shared effects of the average speed inherent in the secondary parameters do not pollute the model. Therefore, it is useful to remove cross-coupled terms from the regression terms. This can be done by using the output window illustrated in Figure 3.3.

Following deletion of cross-coupled terms and higher order terms in the secondary parameter, the model is as shown. Figure 3.7 shows the effect of fixing the secondary parameter similar to Figure 3.6, and Figure 3.8 shows the projection of the model as was shown in Figure 3.5.

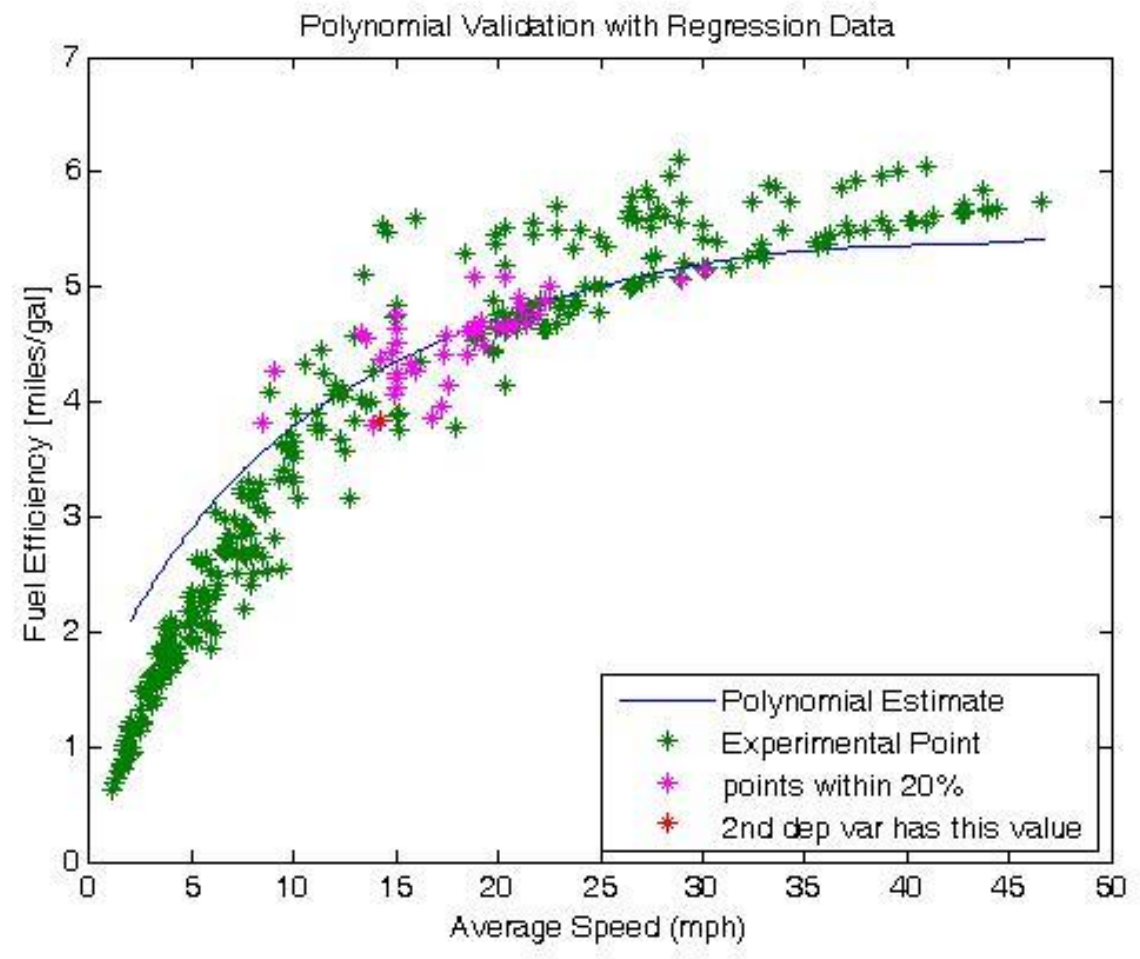

Figure 3.7: Model plotted using a fixed value for the secondary parameter following regressor deletion 


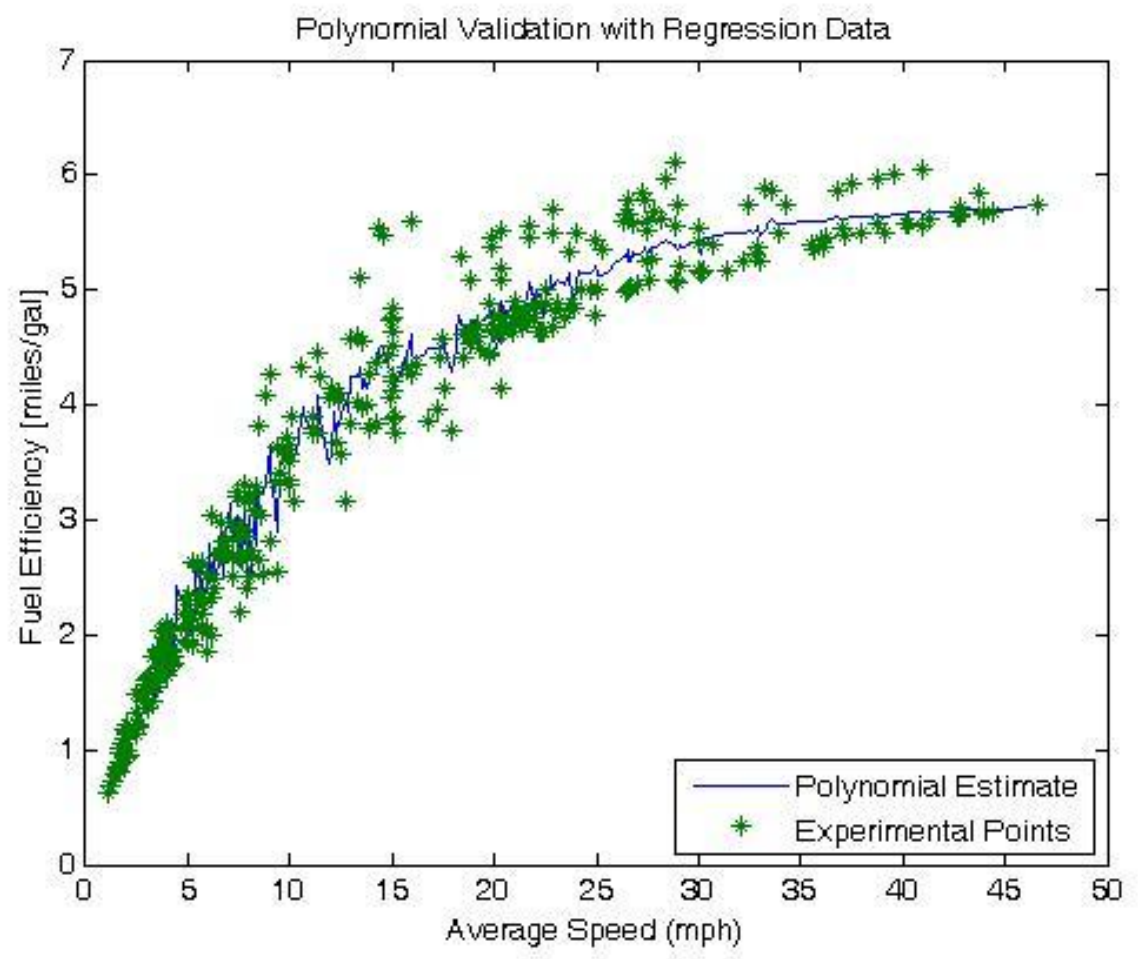

Figure 3.8: Projection of the model using average speed and stops per mile

It is evident that the model is a better fit overall and is less prone to distortion due to small variations in the secondary parameter for a given average speed. Additionally, it should be noted that this method is used in lieu of a numerical evaluation since emissions behavior is generally affected by internal operating characteristics (which are generally not addressed in this approach) and certain intuitiveness is needed as to evaluate the fitness of a model. 


\section{Chapter 4 \\ Interactive Computational Tool for Driving Cycle Generation Based on Genetic Algorithm Optimization}




\subsection{Algorithm Development}

In the biological world, organisms must struggle for survival. These individuals may undergo random changes in their genetic makeup which may alter their structural and functional characteristics (genotype and phenotype, respectively). When these changes are beneficial to the organism, it is better equipped to adapt and survive environmental conditions. As a result, the organism will live longer or more harmoniously and will have a better chance of reproducing and transferring its modified genes to successive generations. Thus, it stands to reason that there will be a higher probability of these genes being present in a given population. This will lead, after several generations, to nearly all members of the population having the new "good" genes. This optimum is, however, temporary; since the environmental conditions are nearly always in a state of change.

The GA attempts to mimic this process by using a set of parameters that are to be determined to maximize or minimize a multiple objective function. This set of parameters represents a possible solution to the problem. This set can be correlated to the individual in the biological world. Taxonomically speaking, this set, is referred to as a chromosome, differing from biology wherein an individual typically has many chromosomes. Additionally, the environment is represented by the multiple objective function: the "fitness function."

The GA begins by evaluating the population of individuals using the fitness function. Individuals having the best fit are assigned a better chance of reproducing. From this assignment, "parents" are selected for reproduction. As in nature, it is unreasonable to allow only the best parents to reproduce following the logic that good parents can produce bad children and vice versa. During reproduction, alterations to the chromosome take place in the form of genetic operators, which are analogous to natural phenomena. Once reproduction is completed, the process begins anew with population evaluation. This continues until a specified number of iterations have taken place or an imposed criterion of convergence is met. These criteria should be defined based on the fitness function; for example, when the rate of improvement is very low from generation to generation over $\mathrm{x}$ number of successive iterations.

In terms of the genetic operators, the changes that a chromosome undergoes are statistical; the probability that a change will result is a fixed, predetermined percentage. Meaning that, for example, only $2 \%$ of individuals, statistically, will undergo alteration. Which 
individuals are selected, however, is completely random. The following flow chart (Figure 4.1) outlines a general Genetic Algorithm.

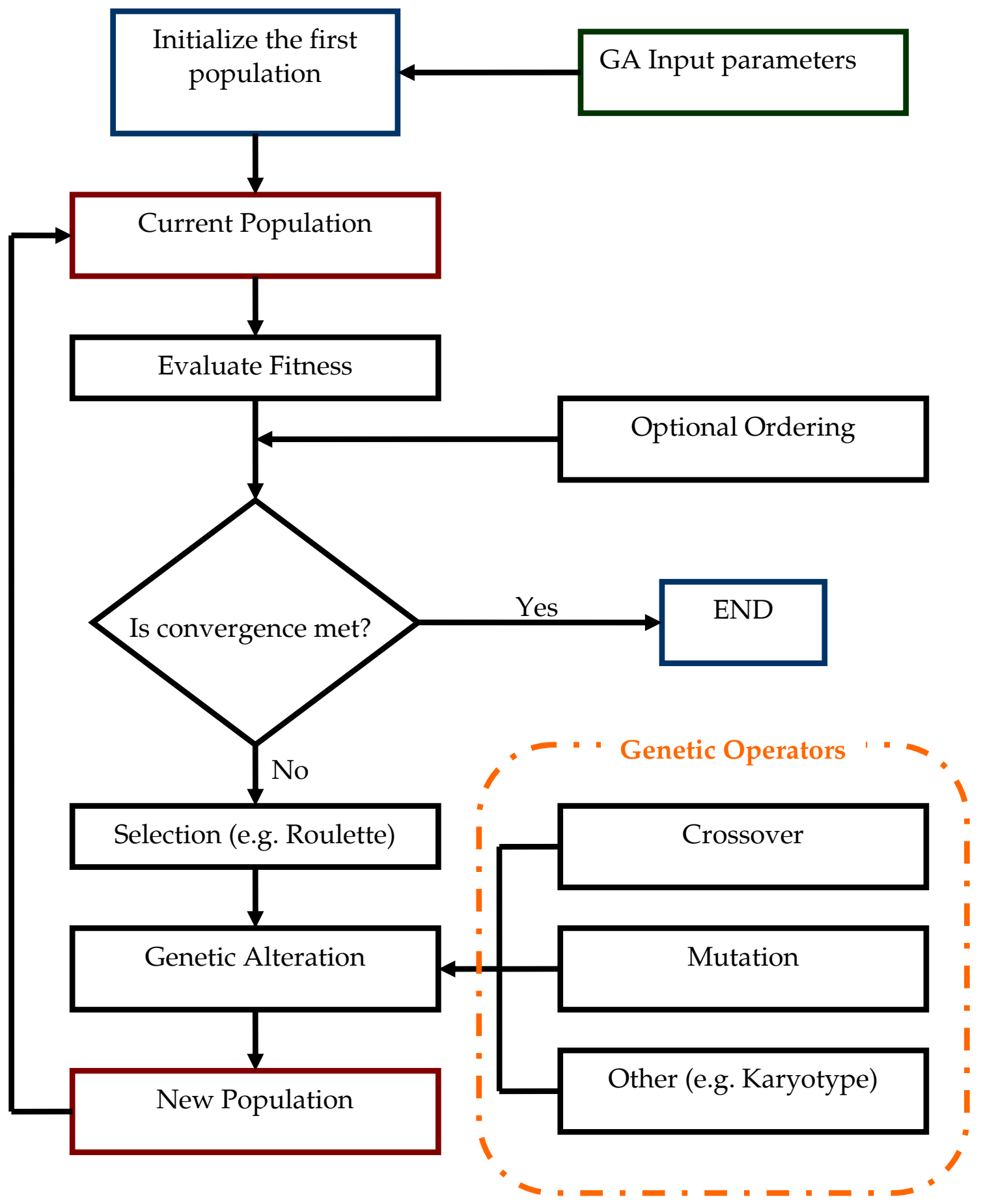

Figure 4.1: Flowchart outlining a general Genetic Algorithm 
The Genetic Algorithm is generally simple, yet credible optimization tool. The process begins with generating random population of genes each consisting of several chromosomes. For simplicity, the terms used in identifying parts of the algorithm have retained the nomenclature of that of the biological system. Genes are the building blocks of each individual. The individuals are generally constructed of binary strings, each gene being either a 1 or 0 . This is not a requirement. Chromosomes can be of any structure, however, the chromosome or individual must be constructed of genes. The general structure of the gene is often representative of certain aspects of the solution.

The onset of the GA process often relies on randomness since the solution is nearly nonreliant on the initial conditions. The population is randomly generated. The size of the population is largely user determined; however, computational resources often play a role in the scope of the simulation. Once the population is constructed each gene is evaluated. This results in a performance index. The performance is based on a user defined evaluation; for the basis of this tool, this is a weighted average of the different inputs. Following evaluation, individuals are selected to be altered. There are several choices for selection methods.

Perhaps the most popular selection method is the Roulette-Wheel method. This is a fitness-proportionate selection method. Each member of the population is assigned a slice of the roulette wheel. The better individuals are given a larger slice of the wheel. The wheel is spun and the individual whose number is spun moves into the next generation. This ensures that the better individuals are given a better chance to succeed, but does not limit the search space in that poor individuals can be introduced to the next step.

Other selection methods include Rank and Tournament Selection. Rank selection selects based on the ranking of individuals rather than absolute differences. This limits the search space by not allowing strong individuals to gain dominance. Tournament selection takes subgroups from within the population and has them compete with each other. The winner is chosen to move to the next generation.

Following the selection step, the genes are altered. The two mostly used types of alteration are mutation and crossover. Mutation is simply altering a single gene within the chromosome. In the case of binary genes, a 1 is changed to 0 or vice versa. When the gene is an integer, a small multiplicative delta is applied to the chromosome. Additionally, when different structures of chromosomes are employed, care must be taken not to invalidate the fitness 
evaluation by exceeding the limits of the chromosome. Additionally, a phenomenon that occurs in nature that could be beneficial to the GA is the addition or deletion of genes, another form of mutation. Simply add a randomly generated gene within an individual or deleting one.

Crossover is directly related to reproduction within a biological system. When two organisms mate, their genes are shared with their offspring. In the GA, the crossover method selects a point from which the preceding genes from chromosome A and combined with the following genes from chromosome B. Also the same combination is employed with remaining genes. This creates two new individuals in the new generation.

In addition to the pervious methods an elitist strategy is often useful. The elitist strategy simply means that the best fit members are automatically added to the new generation. This ensures the most fit members of the population are guaranteed to appear in subsequent generations. Generally, elitism should not be employed as the only selection method, as this would focus the search to local maxima, instead the single best individual is passed into the next generation as to ensure the performance of the best individual is maintained.

Whether alteration consists of all or some of these methods, the individuals to which alteration is to be preformed must be chosen. In nature, not every individual will mate with another. Therefore a percentage of the population of who will mate should be chosen by the user. Similarly, mutations occur in a small portion of a population. The user defined mutation percentage is typically smaller than the crossover rate of occurrence.

\subsection{Implementation}

The cycle generator tool was developed to create new driving schedules using real data. The principle of the tool is that new cycles can be created by combining segments of data from existing second-by-second experimental data. These segments are referred to as microtrips. A microtrip is defined as a period between idles. Acceleration marks the beginning of a microtrip and the end of the previous trip. Figure 4.2 below shows an example of a microtrip defined within the NYBUS cycle. When second-by-second emissions and fuel consumption data are available, it stands to reason that these trips can be reordered and duplicated to change the overall characteristics of the new cycle. 


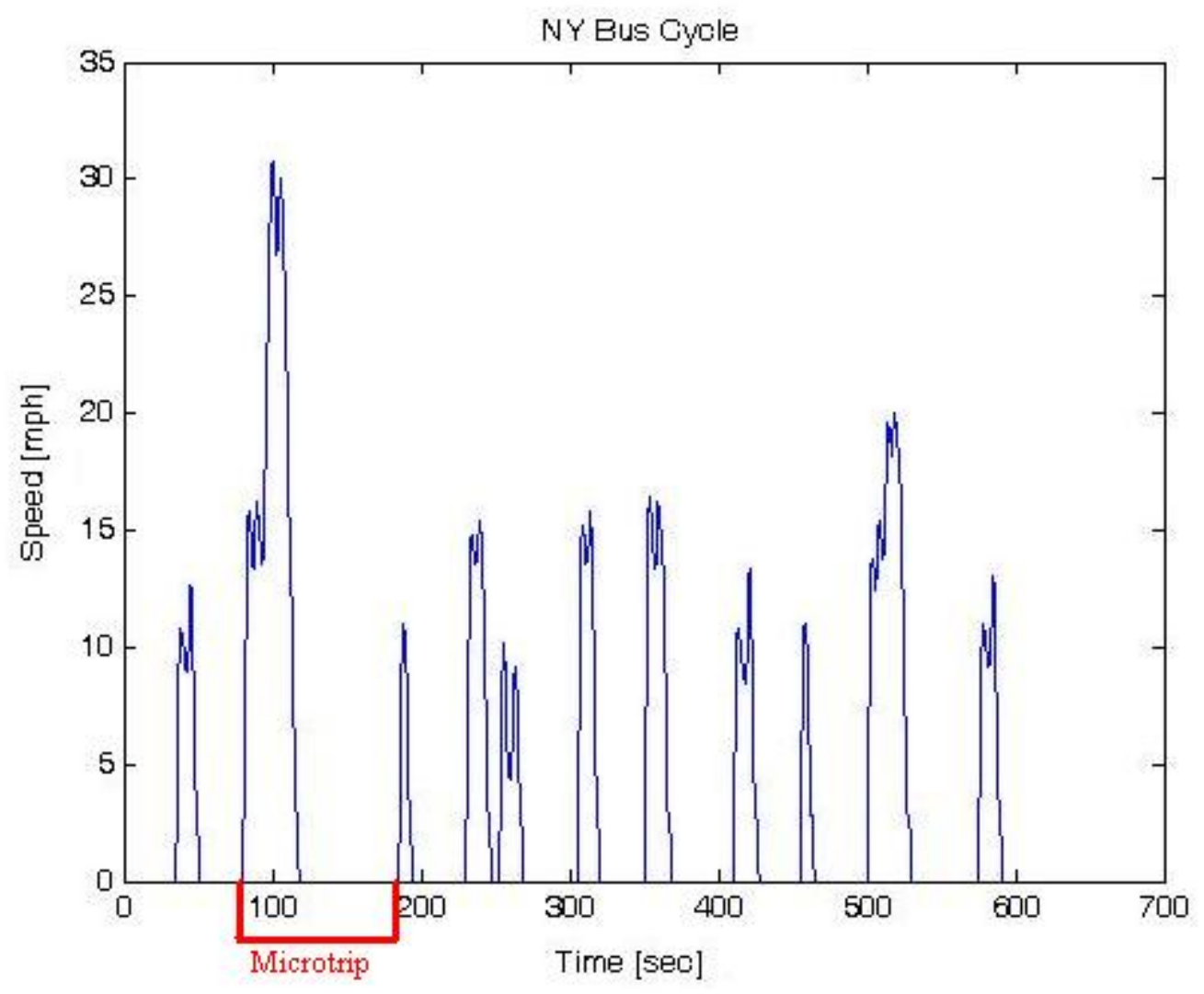

Figure 4.2: Microtrip definition in the NYBUS cycle

The microtrip database was created using trips from 12 standard cycles. The actual time trace of the cycle was aligned with the second-by-second data from the WVU database. After the data were aligned (corrected for time delay) the cycles were broken into microtrips.

For the basis of emissions modeling, average speed, standard deviation of speed, number of stops per mile, percentage idle, and kinetic intensity were chosen to indicate the operating characteristics responsible for the resulting emissions and fuel consumption. The goal of the tool was to fill in gaps in the test data used in the regression tool; therefore the performance index, or fitness function, was defined as a weighted average of each of the driving characteristics errors. The GA rearranged the microtrips in such a way that optimized the order to create a cycle that closely matches the desired driving parameters.

The GA tool employed Roulette-Wheel Selection as well as Elitism, such that the best individual was preserved in the next generation. In addition to crossover and mutation, both single chromosome alteration and chromosome addition/deletion, were used in the tool. The addition and deletion of chromosomes will be referred to as Karyotype alteration. 
The chromosome consisted of a variable number of genes, each gene corresponding to a microtrip. The order of the genes within the chromosome was an order in which the microtrip succeed each other in the newly generated cycle. A gene was a three integer structure. The first integer was an index marker pertaining to one of the twelve standard cycles, and the subsequent integers were time markers for the respective beginning and end of a microtrip within the given cycle. An example of this structure is shown in Figure 4.3. This structure of the microtrip was chosen because it lends the newly created cycles to more flexibility. This flexibility occurs because new second-by-second emissions data does not need to be modified, excepting time alignment, since the model references time bands within a given standard cycle. This proved useful when moving between vehicle configurations and fuel types; given that the emissions data is represented in one or more of the twelve cycles.

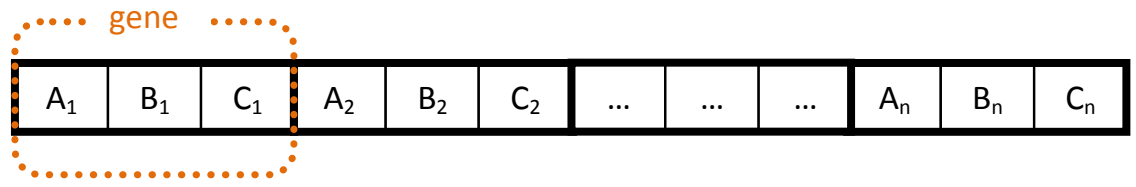

A: cycle identifier B: microtrip start time C: microtrip end

Figure 4.3: Example individual structure (real representation)

For each iteration, the microtrips were concatenated into a single driving cycle and then evaluated as per the performance index. The algorithm continued until the designated number of generations has been computed, this replaced a convergence criterion. The results were then displayed for the user. These results include the index of the selected individual driving cycle, the values of both the target and achieved parameters, the evaluated performance of the overall cycle, and a time history plot of the newly created cycle.

At this point the user can continue with additional generations. This feature is useful when a user lacks the computing power to perform large numbers of iterations at a time. The user is able to break large numbers of iterations into a few smaller sets of iterations and still achieve the total number of iterations that they desire.

The algorithm for genetic optimization of newly created driving cycles is reliant upon parameters defined by the user. Primarily, the user is responsible for defining the input parameters which will define the output cycle. These are selected from the following: average 
speed with idle, standard deviation of speed with idle, number of stops per mile, percentage idle, and kinetic intensity. These parameters are chosen such that they align with the polynomial tool. Each of the user defined inputs carries a respective weight indicative of its importance to the output cycle. Additionally the user can modify the default parameters regarding the number of iterations, size of the initial population, length (in terms of number of microtrips) of the cycle, and maximum relative errors for each input parameter.

Once the user initializes the tool, the mutation and crossover rates for the simulation are selected. Additionally a Karyotype alteration rate is defined; this governs the likelihood that a chromosome will change in length. Biologically speaking, a Karyotype alteration can be approximated to a genetic mutation, and as such should have a lower occurrence rate than crossover. While these rates can be easily changed, they are not generally accessible to the user. The rate for mutation, whether classically defined or Karyotype, is initialized to a much smaller rate than the crossover due to the issue of exploration vs. exploitation. Generally, exploration is a longer process and exploitation is more immediate. More specifically exploitation refers to the tendency to focus on what is working and exploration looks to what may work. It is necessary to balance exploitation and exploration in a GA since imbalance can lead to premature convergence.

Following initialization, the initial population is created by randomly selecting microtrips and aligning them into individuals of a predefined length. Next, each individual is evaluated by concatenating the microtrips and computing the relative error of that newly created cycle's driving parameters to those specified as the desired output by the user.

$$
\text { error }=|(A-B) . / B|
$$

where $A$ is a vector of driving parameters computed from the newly generated cycle, and $B$ is a vector of target driving parameters defined by the user.

Additionally the error is computed using element wise division.

Next the error is checked as per the user defined maximum relative error for each input parameter. This value is computed as follows:

error $_{\text {val }}=-1 . /$ error $_{\text {input }} *$ error +1 
Where error ${ }_{\text {input }}$ is the user defined values for maximum relative error

and error is the computed relative error.

All division and multiplication is element wise.

Also, if err_val is less than zero, err_val is set to equal zero.

Eq. 4.2

Next, the performance index for the individual is calculated as a weighted average of the computed error for the individual as shown below:

$$
p f=\frac{\varsigma r r_{-} v a l^{*} w t^{-}}{\sum w t}
$$

Where wt is the user defined weighting vector. If $p f=0$ then set $p f=0.001$.

This process is repeated for each individual in the population.

Once each individual has been evaluated, the individuals are subjected to genetic alterations and roulette wheel selection in order to produce a new population. First the roulette wheel method is used. The Roulette-Wheel method works by evaluating the probability of selection for each individual based on its performance index, then computing the cumulative probability. The first step is to compute the fitness of each individual and the total fitness of the population $T F$.

$$
T F=\sum_{i=1}^{n} p f_{i}
$$

Next, the probability of selection $p$ for each individual is computed.

$$
p_{i}=\frac{p f_{i}}{T F}
$$

Eq. 4.5

Then the cumulative probability $q$ is calculated for each individual as follows:

$$
q_{i}=\sum_{j=1}^{i} p_{j}
$$


Eq. 4.6

Finally the roulette wheel is "spun" resulting in a random $1 \mathrm{x} \mathrm{n}$ matrix, where $\mathrm{n}$ is the number of individuals in the population. The new population is determined by selecting individuals determined by the spin $\mathrm{r}_{\mathrm{j}}$ and the cumulative probability $\mathrm{q}_{\mathrm{i}}$. If $r_{j}<q_{i}$ then select the first individual, otherwise you select the individual that satisfies,

$$
q_{i-1}<r_{j} \leq q_{i}
$$

where $\mathrm{j}$ is $1: n$.

Eq. 4.7

After roulette selection, genetic alterations are preformed. The Karyotype genetic operator is applied first. An individual is selected when the Karyotype alteration rate exceeds a randomly generated number for an individual. Then a gene (microtrip) in the individual is selected, at random, to be altered. Next, the algorithm randomly decides whether to add or delete the gene. Addition and deletion have equal probability of occurring. Additionally, should the individual be comprised of only one microtrip the trip will be replaced with a new microtrip rather than deleted. Figure 4.5 shows an example Karyotype alteration for an individual. For illustration, the microtrips are defined as a binary vector, as shown in Figure 4.4.

\begin{tabular}{|l|l|l|l|l|l|l|l|l|l|l|l|l|l|}
\hline 1 & 0 & 1 & 0 & 1 & 0 & 1 & 1 & 0 & 1 & 1 & 1 & 0 & 1 \\
\hline
\end{tabular}

Figure 4.4: Example individual structure (binary representation) 
The gene is removed, or a new gene is added after

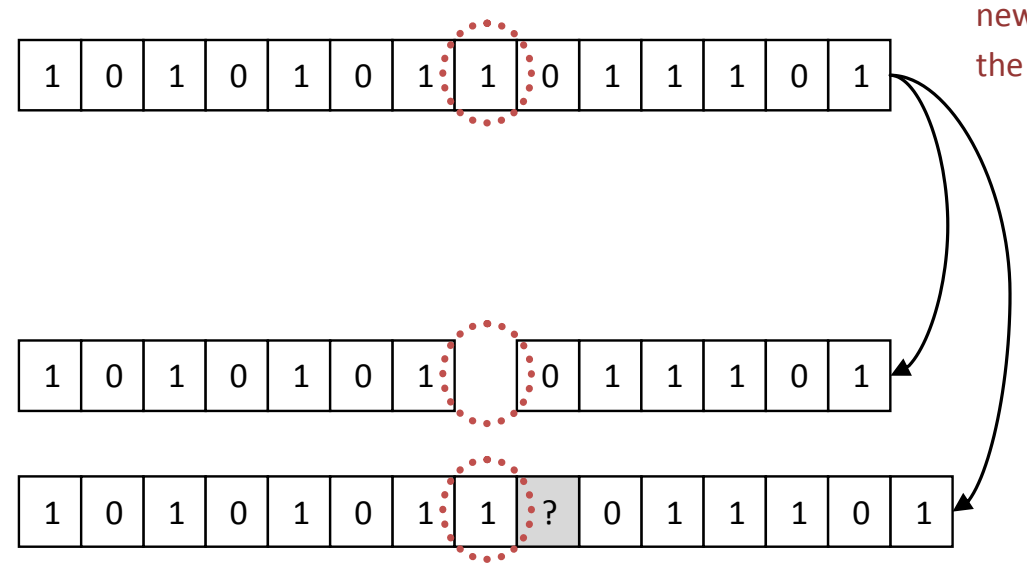

Figure 4.5: A gene is selected for Karyotype alteration and is either deleted or a new gene is added as per the algorithm

Following Karyotype alteration is mutation. An individual is selected for mutation in the same manner as with Karyotype alteration. When an individual is chosen, a gene is selected at random. This gene now will be either replaced with a new microtrip from within the individual, or be replaced with a new microtrip from the microtrip database. Both types of mutation have equal probability of occurrence. Figures 4.6 and 4.7 show example mutation in both binary and real representations respectively.

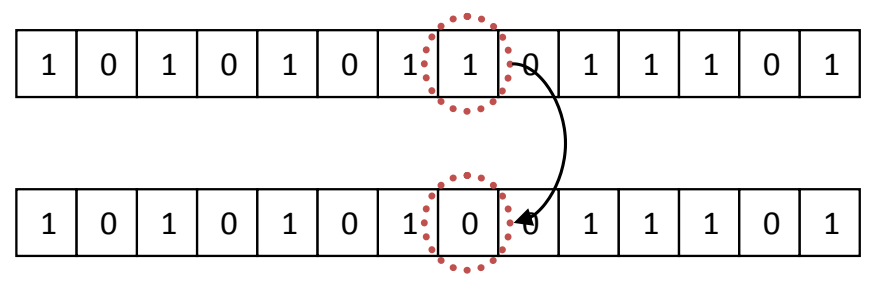

Figure 4.6: Example gene mutation (binary representation) 

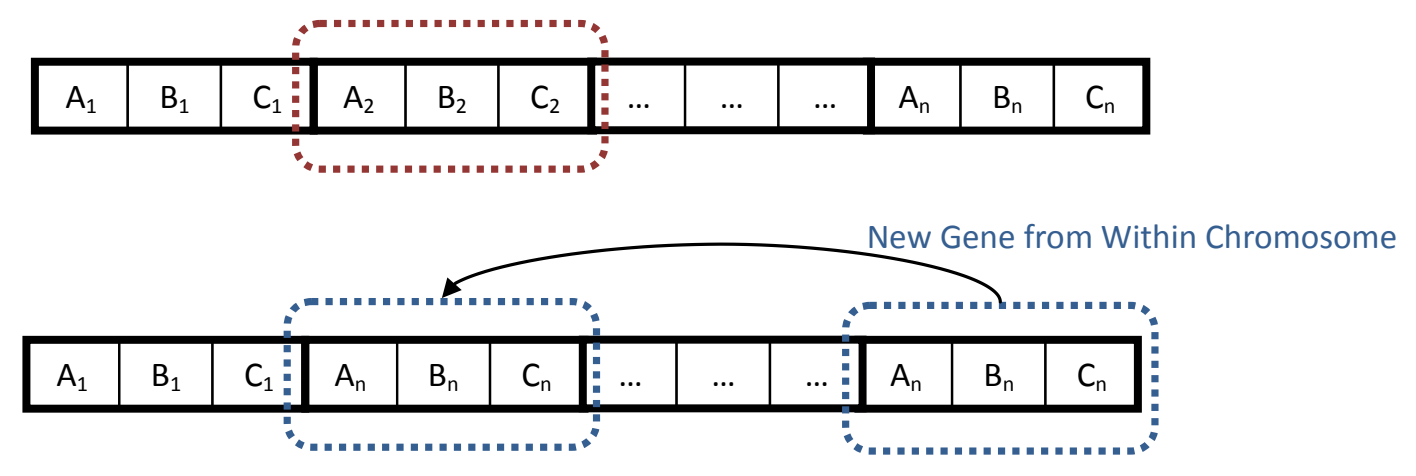

New Gene from Microtrip Database

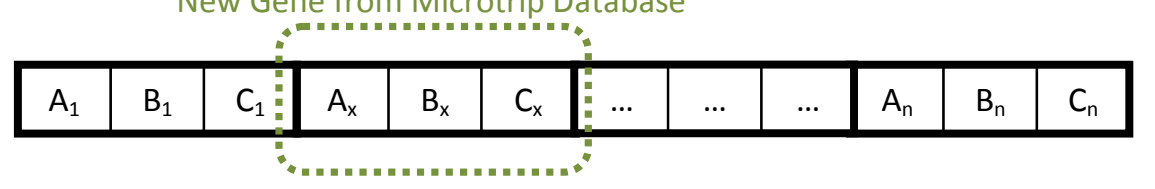

Figure 4.7: Example gene mutation (real representation)

Finally, crossover is performed. Individuals are paired and pairs are chosen to be "mated" following the same procedure as mutation and Karyotype alteration. Pairs that have been selected for crossover are evaluated in length and the larger of the two has a crossover point selected between two genes. The genes from the larger are combined with the smaller using the genes up to the crossover point from the larger individual and the genes from the crossover point of the smaller individual, and vice versa. This results in two new individuals comprised of the preceding pair. Figure 4.8 show an example crossover, where individuals are represented as binary vectors of the same length. 


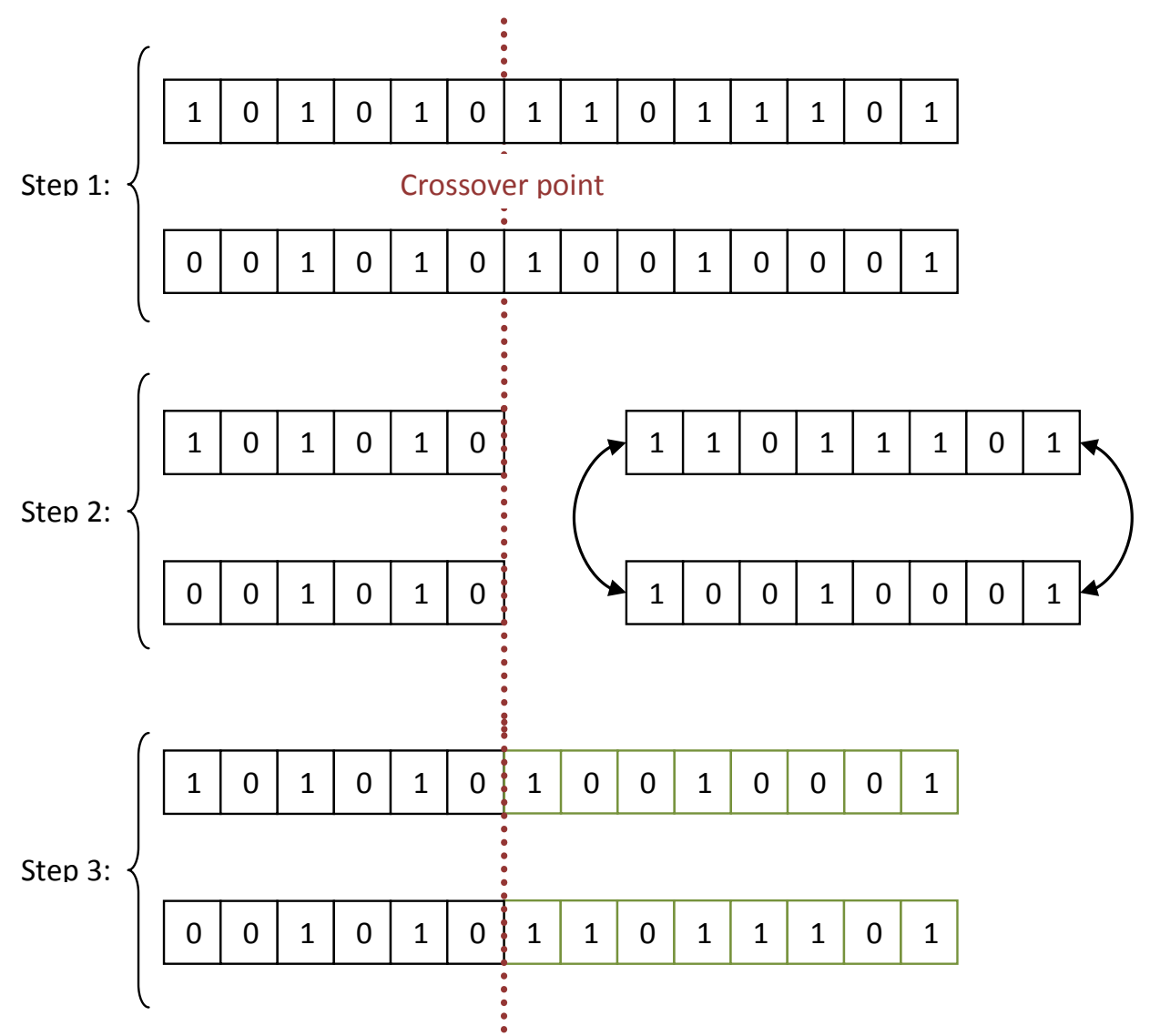

Figure 4.8: Example crossover preformed on a pair of individuals

Once all alterations have been made, the newly generated population repeats the process beginning with performance evaluation.

Similar to the process for the PolyTool, the genetic algorithm requires a graphical interface for usability. The tool opens with a screen that will allow users to define the target driving parameters for the output cycle, as well as the significance of each parameter by defining its weight. Figure 4.9 shows the GUI for the GA tool. 


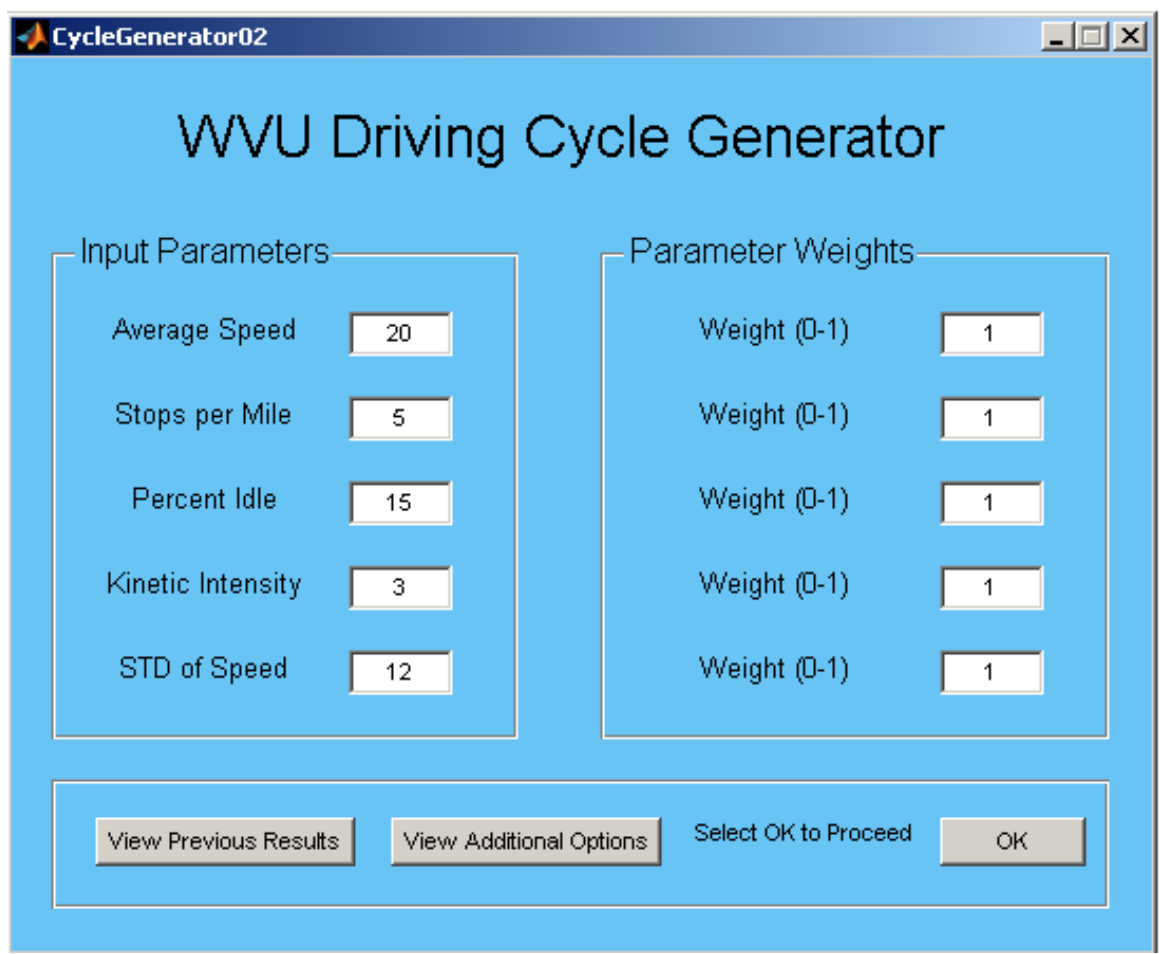

Figure 4.9: Cycle Generator Interface

At this point the user can opt to define optional parameters for the GA tool. These include: number of generations, size of initial population, maximum number of microtrips in an individual, and maximum relative errors for each input parameter. Figure 4.10 shows this option window.

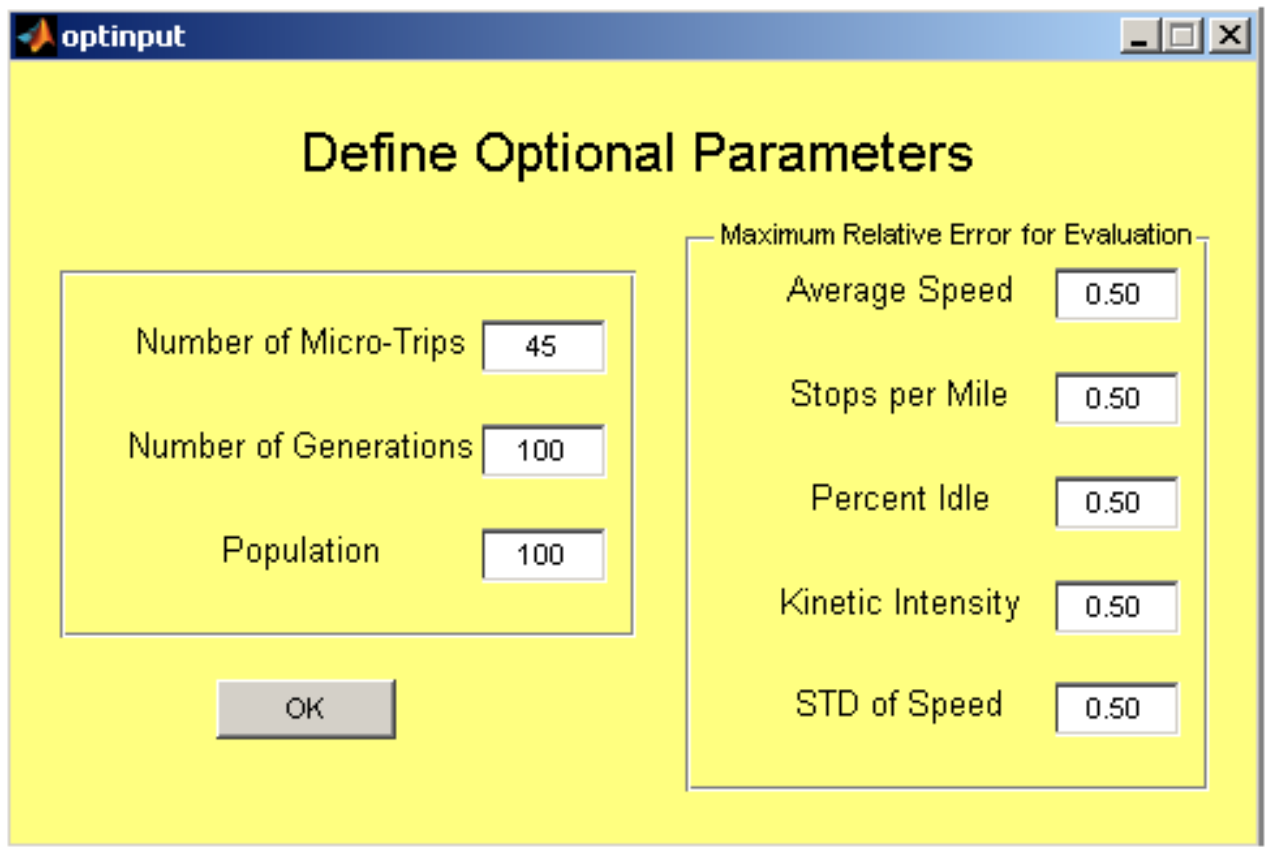

Figure 4.10: Optional user interface window 
Upon conclusion of the simulation, the user is presented with a results window. This window shows the performance history with each generation and a graphical realization of the newly created cycle. Additionally the user is presented with the values of the target parameters and the actual parameters of the newly created cycle. At this point, the user can change the optional parameters and rerun the tool, or they can continue with the simulation with the last population (i.e. add generations). This is particularly useful when the user does not receive results that are to their liking, or when computationally they are unable to run large numbers of iterations. Figure 4.11 shows an example results window.

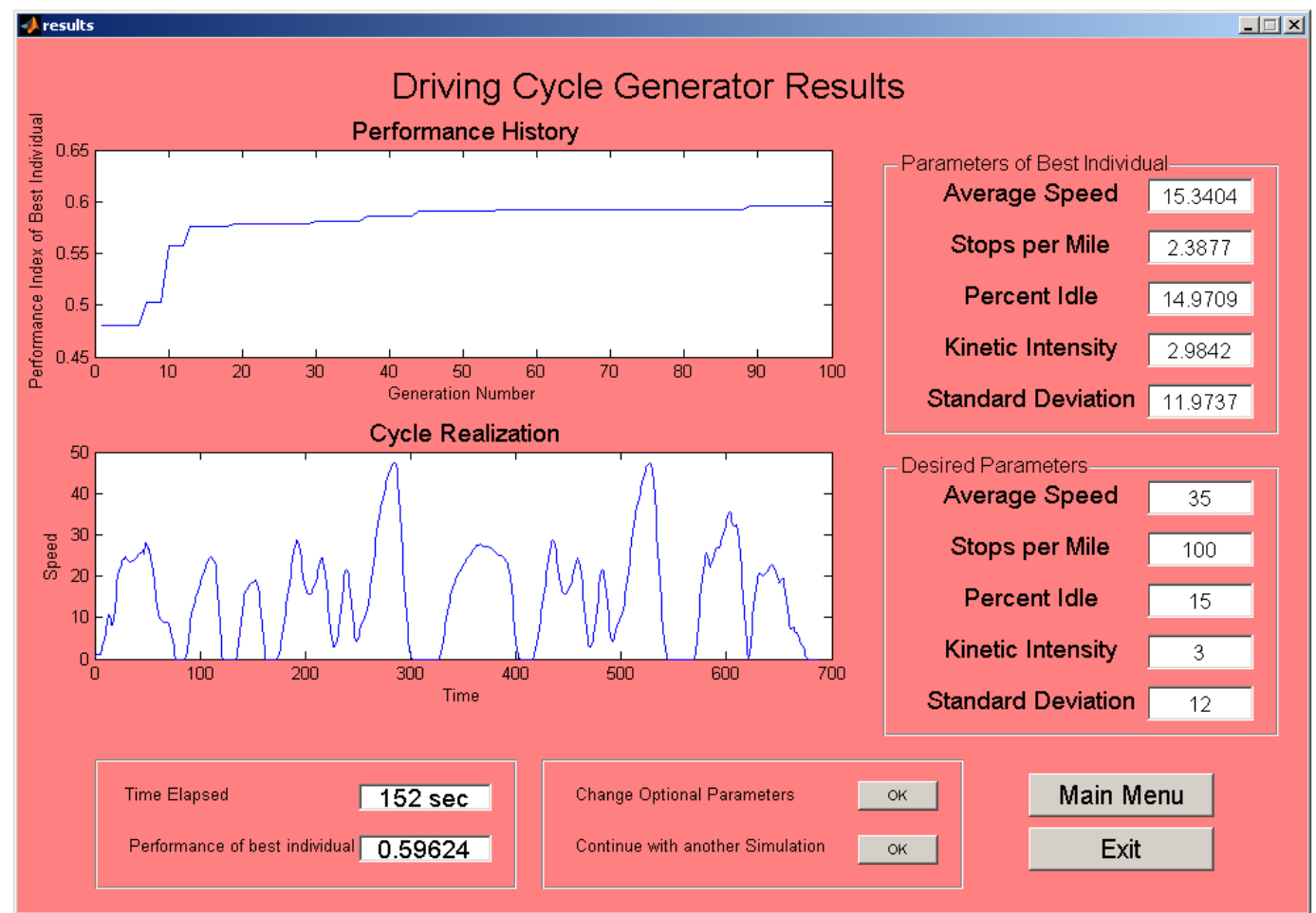

Figure 4.11 Example results window from the GA tool

\subsection{Development of Driving Cycles using the Cycle Generator Tool}

This section serves to illustrate the method for creating new driving cycles using the cycle generator tool described herein. As with the PolyTool, this section serves mainly as an example of obtaining data from the tool. The cycle created here was constructed to meet arbitrary parameters. 
To begin creating a cycle, the user runs the program where they are presented with the window shown previously in Figure 4.9. In this window target parameters desired in the final cycle are defined. In this example, an average speed of $15 \mathrm{mph}, 5$ stops per mile, $15 \%$ idle, and a standard deviation of speed of 12 are chosen. Kinetic intensity is to be omitted (arbitrarily) from this example. Once all data is entered, the weight for $\mathrm{KI}$ is set to 0 . This serves to omit $\mathrm{KI}$ from the generator. It should be noted that while KI is being omitted, the tool will still give a values for it in the results window. Omitting KI simply means that the error of the cycle's KI value does not affect the overall fitness of the cycle to the target parameters.

Following entering the target parameters, optional parameters for the algorithm will be changed. All optional parameters are left as the default values except the number of generations. In this example, the number of generations is set to 50. These parameters are defined in the window as shown in Figure 4.10.

Once all of the parameters are selected, the user will click OK to execute the algorithm. Once the designated number of generations has been computed, the tool displays the results window (Figure 4.12).

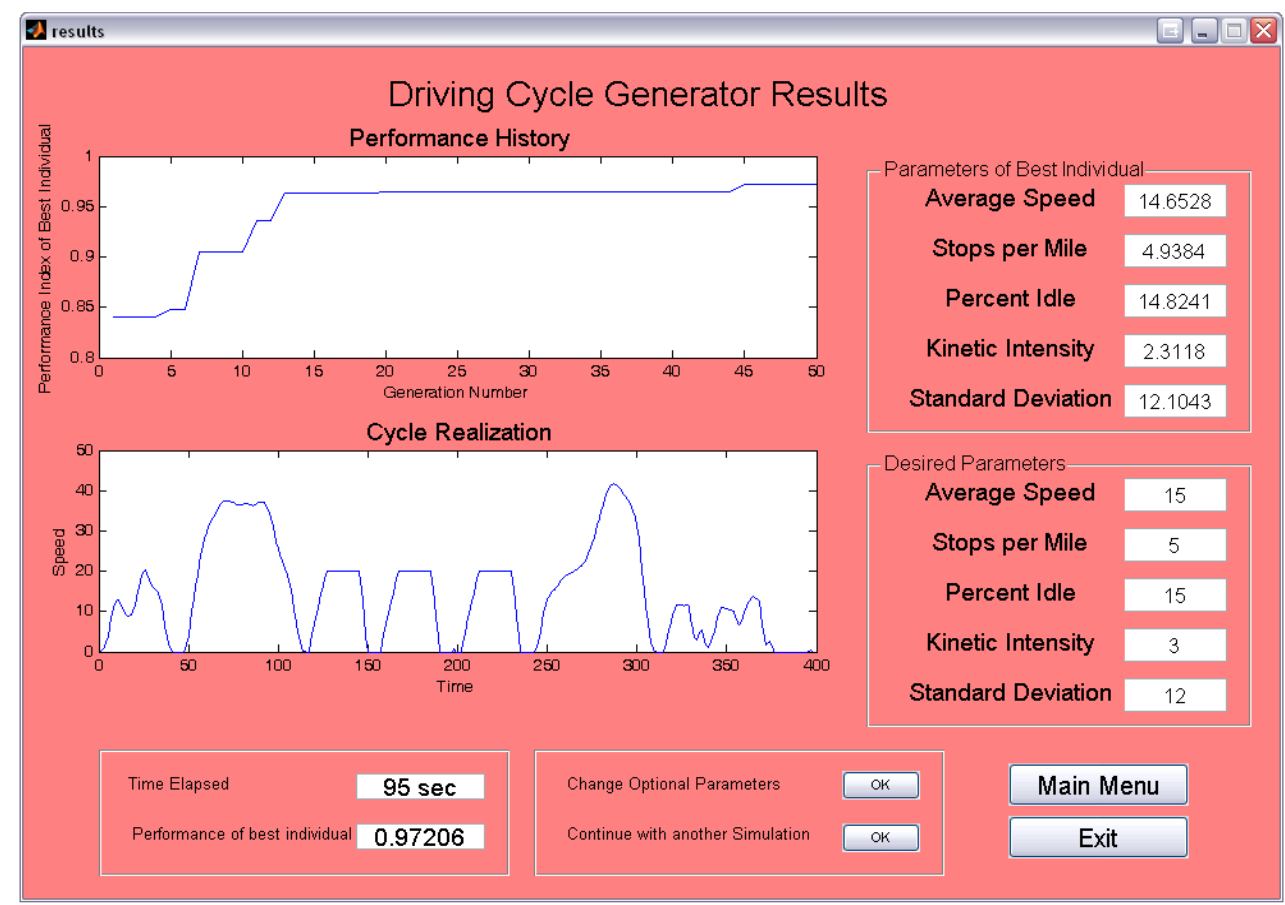

Figure 4.12: Results window for cycle generator 
The results for this example show that the tool is able to create a cycle with a performance index of roughly $97 \%$ and has done so in 95 seconds. In addition to the values of each parameter, the results window displays a realization of the cycle. This is helpful in determining whether or not the cycle that was created is a practical cycle. More specifically, that there is not a microtrip, within the final cycle, that contains erroneous measurements. It is of note that this example obtained good results in few iterations because the target parameters are within rational limits as to the value for average speed. Being that the cycles created with this tool are comprised of actual test data, the tool is unable to obtain a solution for parameters that are unreasonable in real world situations.

As a byproduct of this limitation, the cycle generator also lends insight and allows for the creation of acceptable limits for each input parameter in terms of average speed. These limitations that were deduced and introduced into IBIS are shown in Figures 4.13-16.

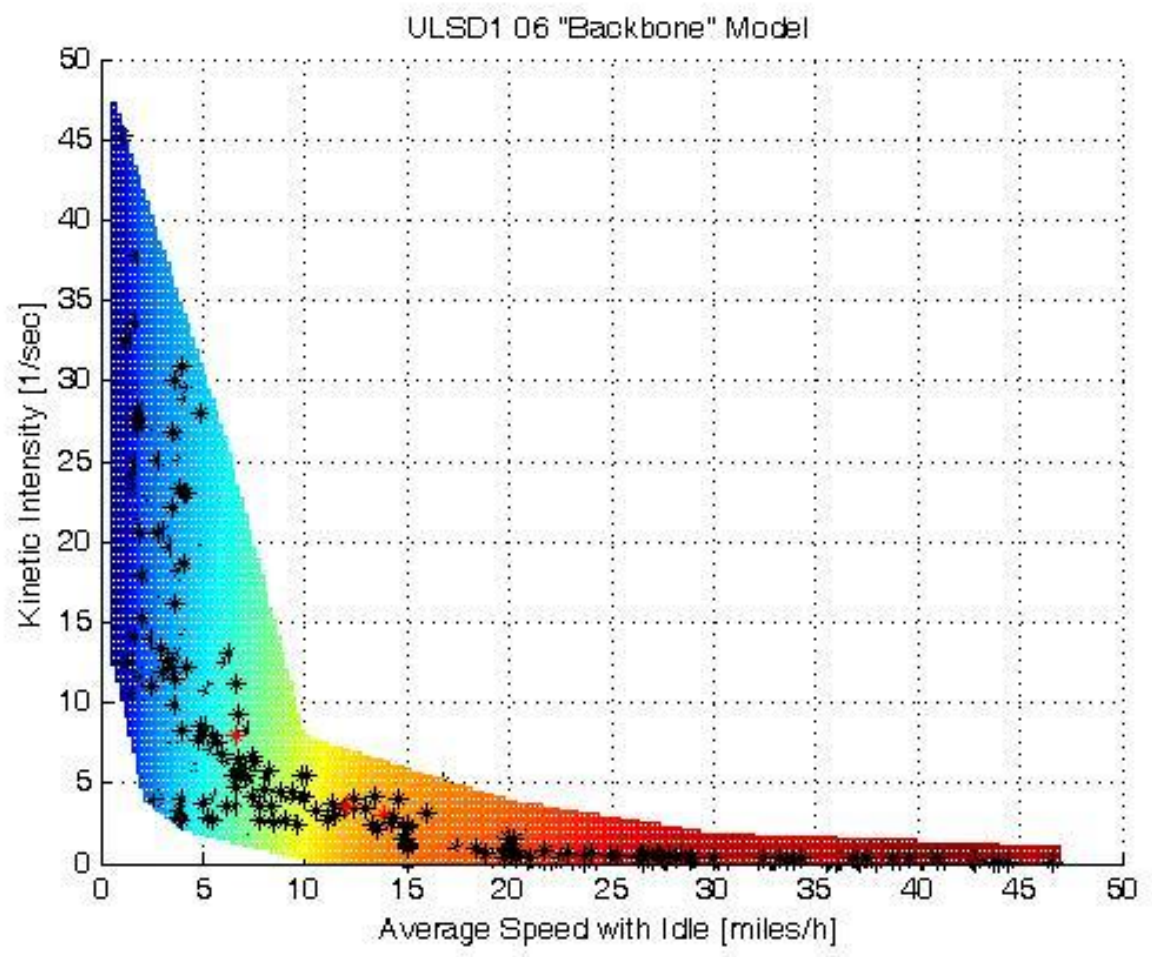

Figure 4.13: Limits on kinetic intensity in terms of average speed 


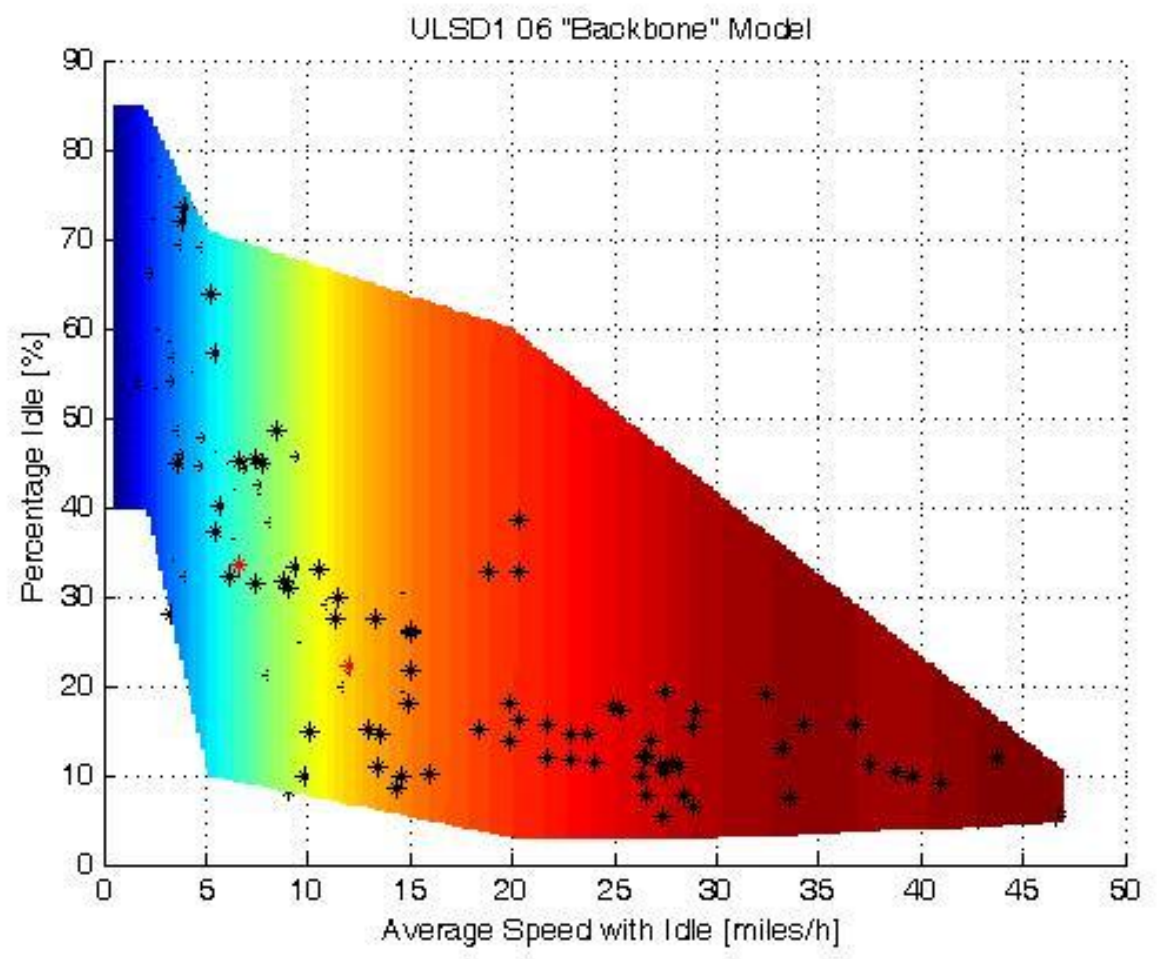

Figure 4.14: Limits on percentage idle in terms of average speed

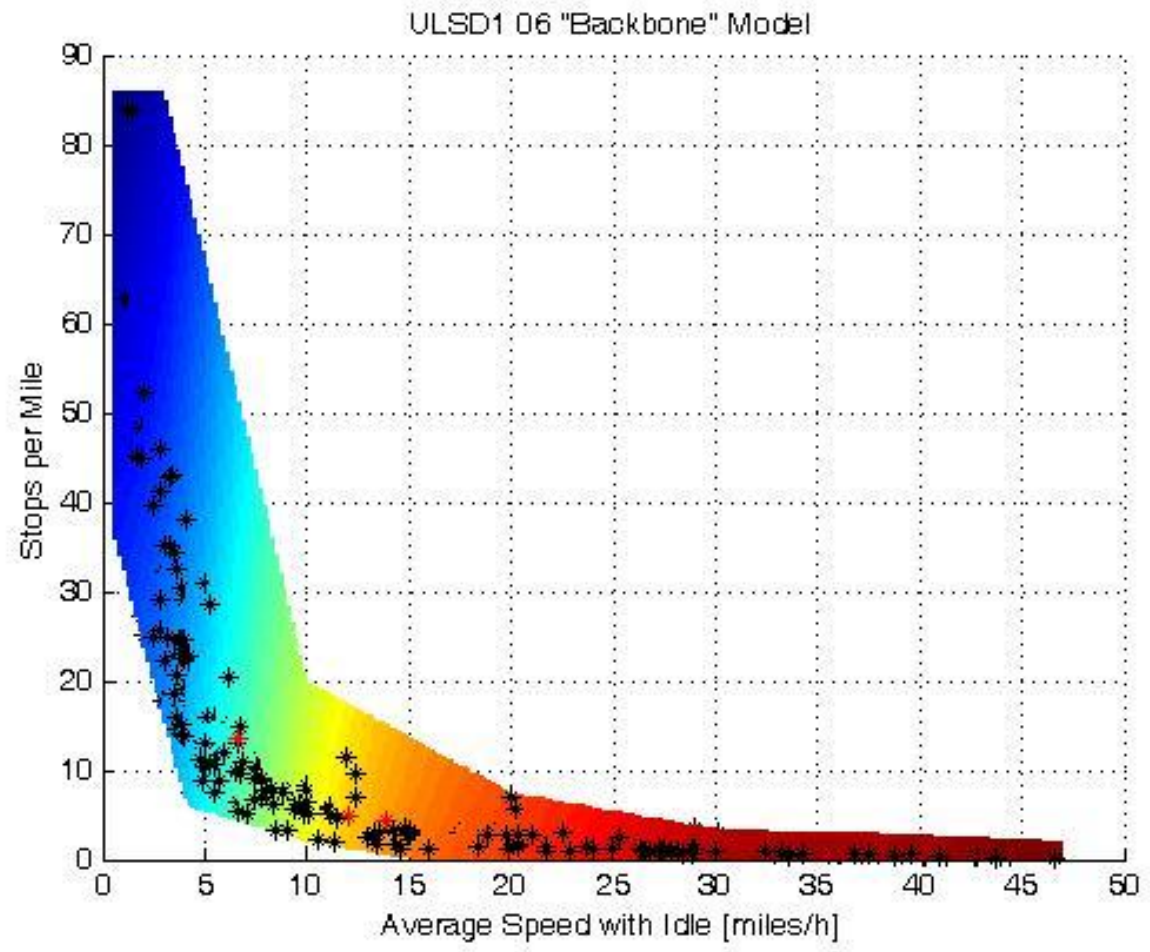

Figure 4.15: Limits on stops per mile in terms of average speed 


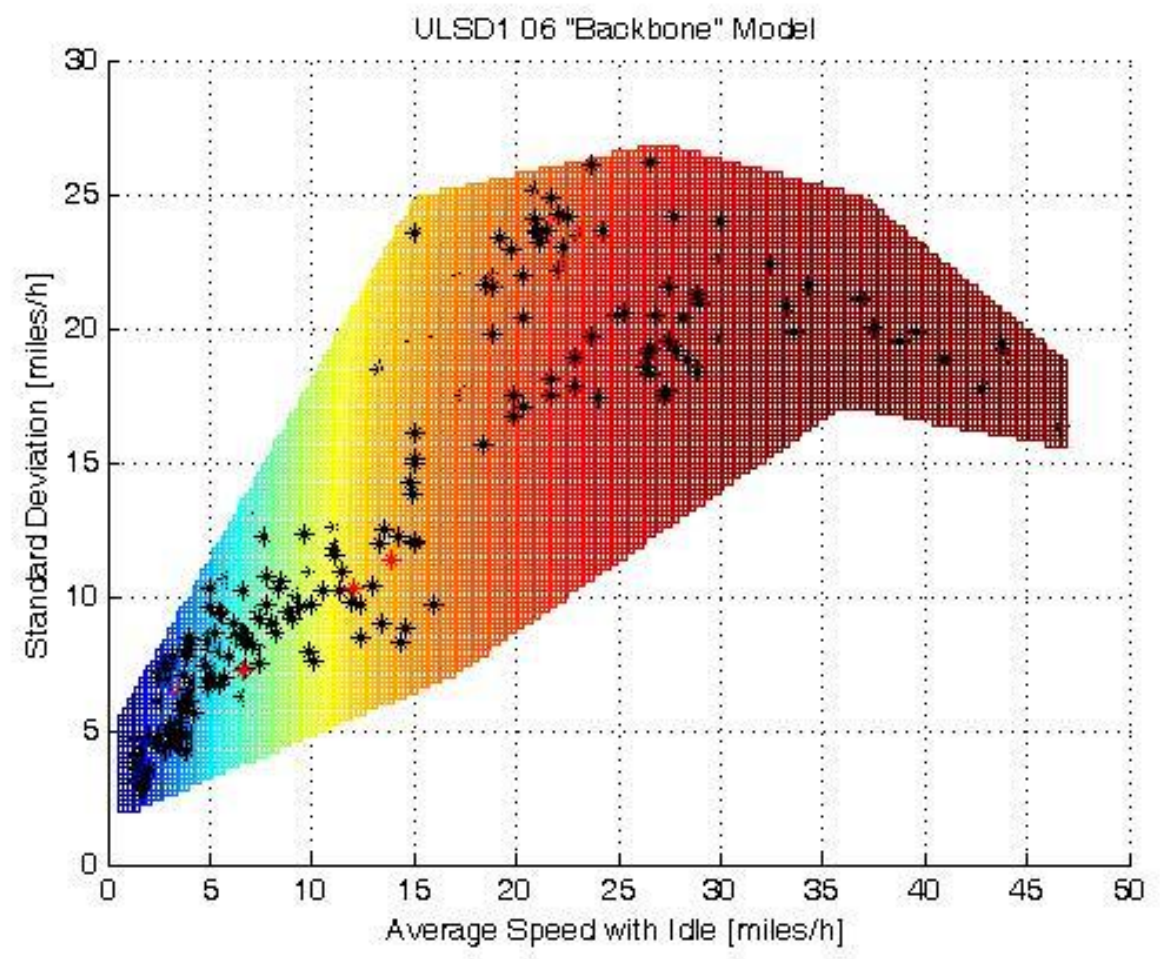

Figure 4.16: Limits on standard deviation in terms of average speed 


\section{Chapter 5 Additional Tools}




\subsection{Model Verification Tool}

An Additional tool has been created to verify each backbone model. The goal of this tool is to provide users with a three dimensional representation for each pair of inputs. The tool is also intended to show the effectiveness of the model in comparison to the experimental data. This tool is useful in identifying areas of the model where repair algorithms are needed. Figures 5.1 and 5.2 show the menus supplied by the tool by which selections are made. The user is able to select from a list of available backbone types: Diesel, $\mathrm{CNG}$, Hybrid. Once the initial selection is mad the user then chooses which emissions species or fuel economy will be displayed.

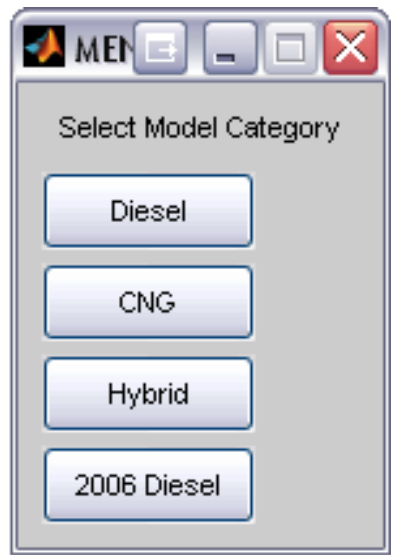

Figure 5.1: Initial menu for the selection of backbone model type

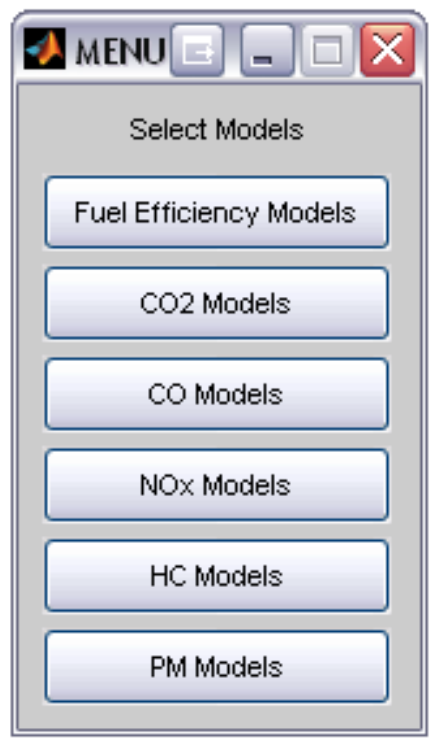

Figure 5.2: Menu for the selection of results output

Once all secetions are completed, the tool displays the specified output as a set of four Figures. The Figures display the models for each pair of polynomial models. Figure 5.3 shows 
an example Figure generated by this tool. This example is a backbone model for a 2006 Diesel Bus with the input parameters of average speed and percentage idle.

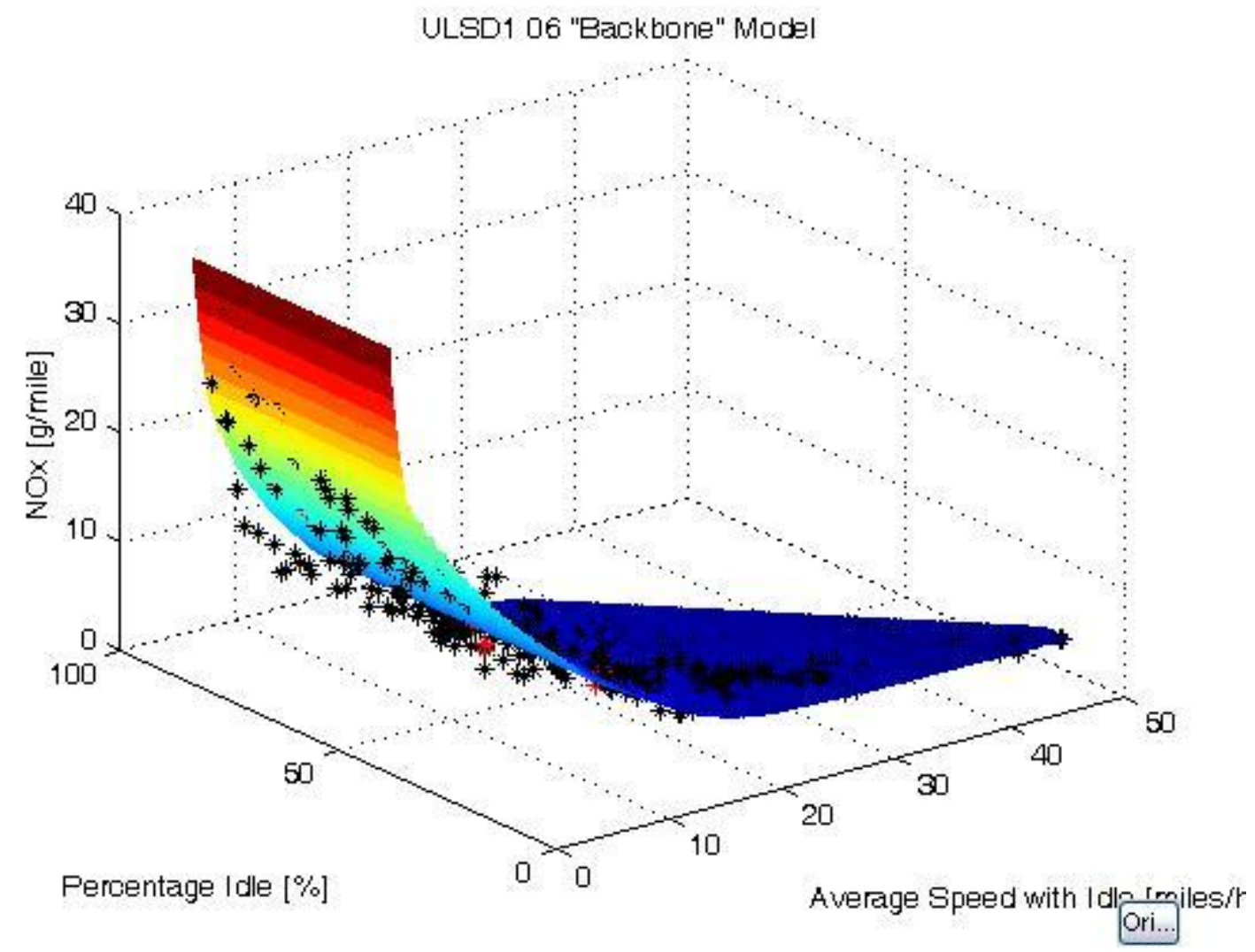

Figure 5.3: Example three dimenseional model supplied by the tool

In this Figure the user may manipulate the viewing angle by using the functionality built into the MATLAB Figure tool bar.

\subsection{Offline IBIS Tool}

For the development of IBIS, it was necessary to develop an offline version of the tool. This was done for several reasons. Since the development tools were created using MATLAB, it stands to reason that the models created could be more easily evaluated in a MATLAB environment. Also, general familiarity with GUI creation and coding syntax promoted MATLAB as the final candidate for this version. The offline tool has been developed in a similar to the implementation of each of the aforementioned development tools.

This tool allows a user to specify each individual vehicle in his/her fleet. Upon starting the tool, the user is presented with the following window: Figure 5.4. 


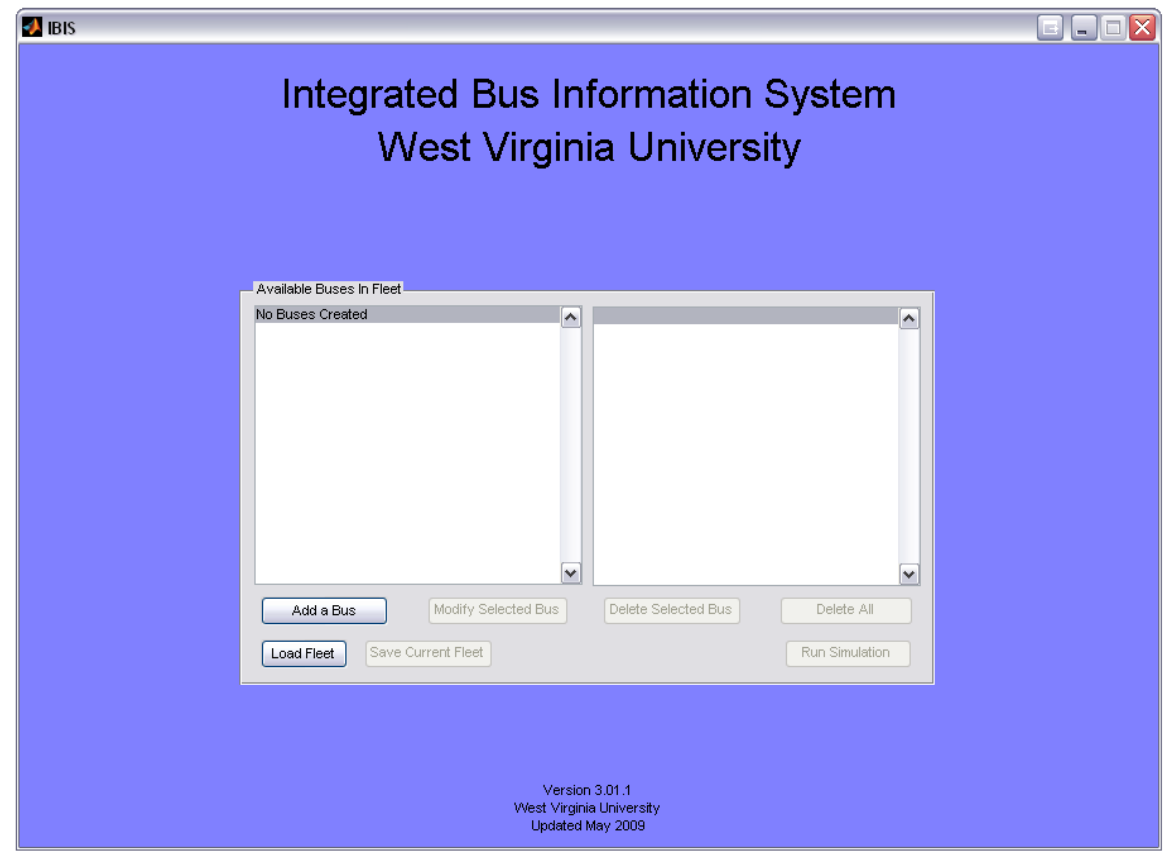

Figure 5.4: Initial window for the offline IBIS tool

In this window a user may opt to load a previously defined vehicle fleet, or begin a new fleet by pressing the appropriate button. When the user selects to add a new bus to the fleet, he/she is presented with the window in Figure 5.5. Here the user defines each parameter governing the operation and architecture of a specific bus in the fleet. The user may also decide to specify overall mileage for this bus. It should be noted, however, that once mileage is entered it must be entered for each additional bus in the fleet. This mileage allows for the calculation of fleet total emission and fuel consumption data. 


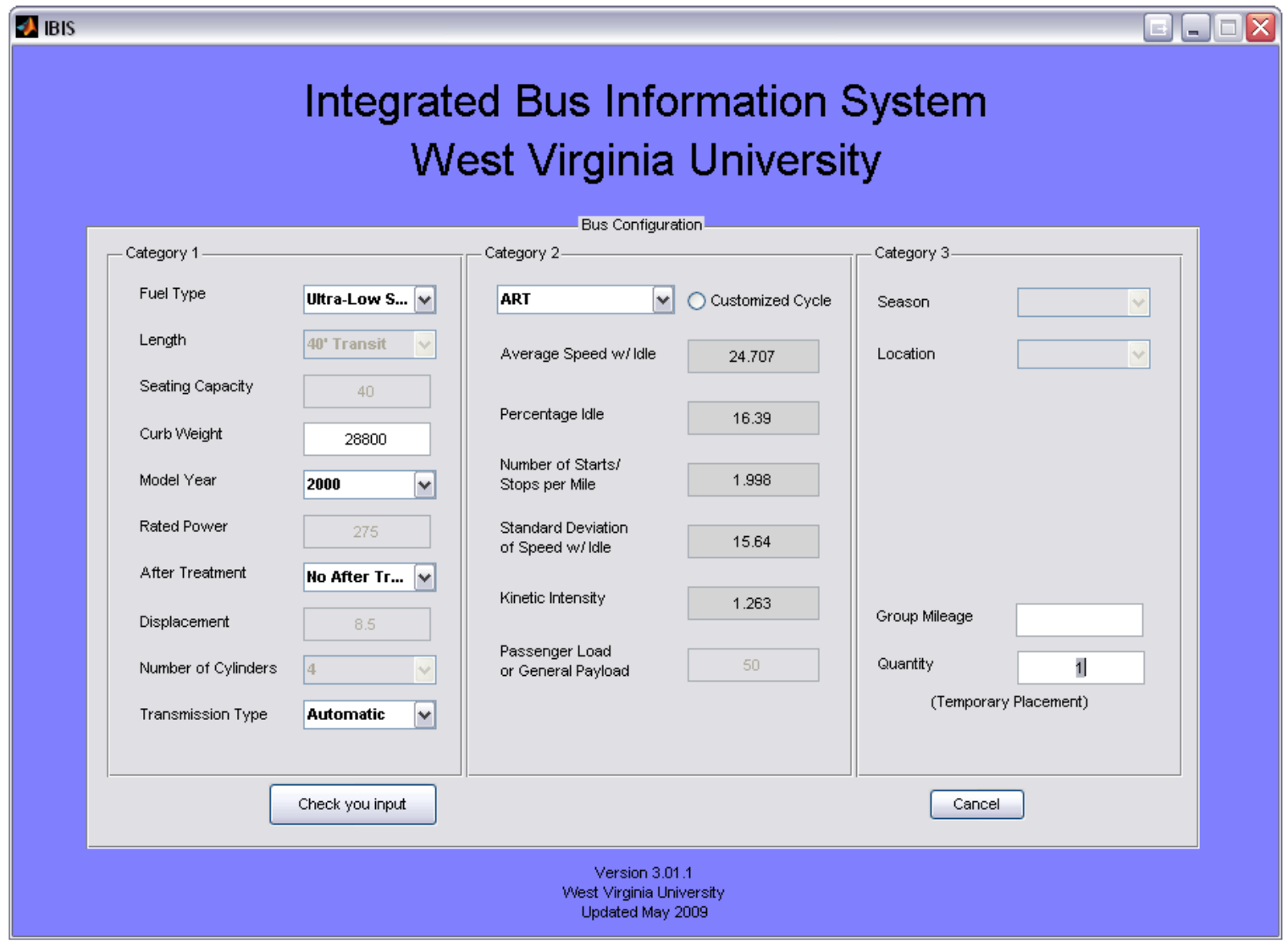

Figure 5.5: Bus design window

Once all selections have been made, the tool checks the validity of each input. For category 2 , the average speed must fall within the range of 2-50 mph. From this designation of average speed, each additional driving characteristic is evaluated via a function of average speed as seen in Figures $4.13-16$.

Once all buses have been defined, the tool evaluates each individual bus based on its design. This included evaluation of the backbone models and the application of any necessary correction factors. Finally the user is presented with the results window as shown in Figure 5.6. In this window the user may select individual bus groups to evaluate, and is presented with fleet outputs. The fleet output is given in grams or gallons per unit time, and in cases of mixed fuel types data is represented in diesel energy equivalents. If mileage for each group is provided, the user is given the option to display fleet results as totals. 


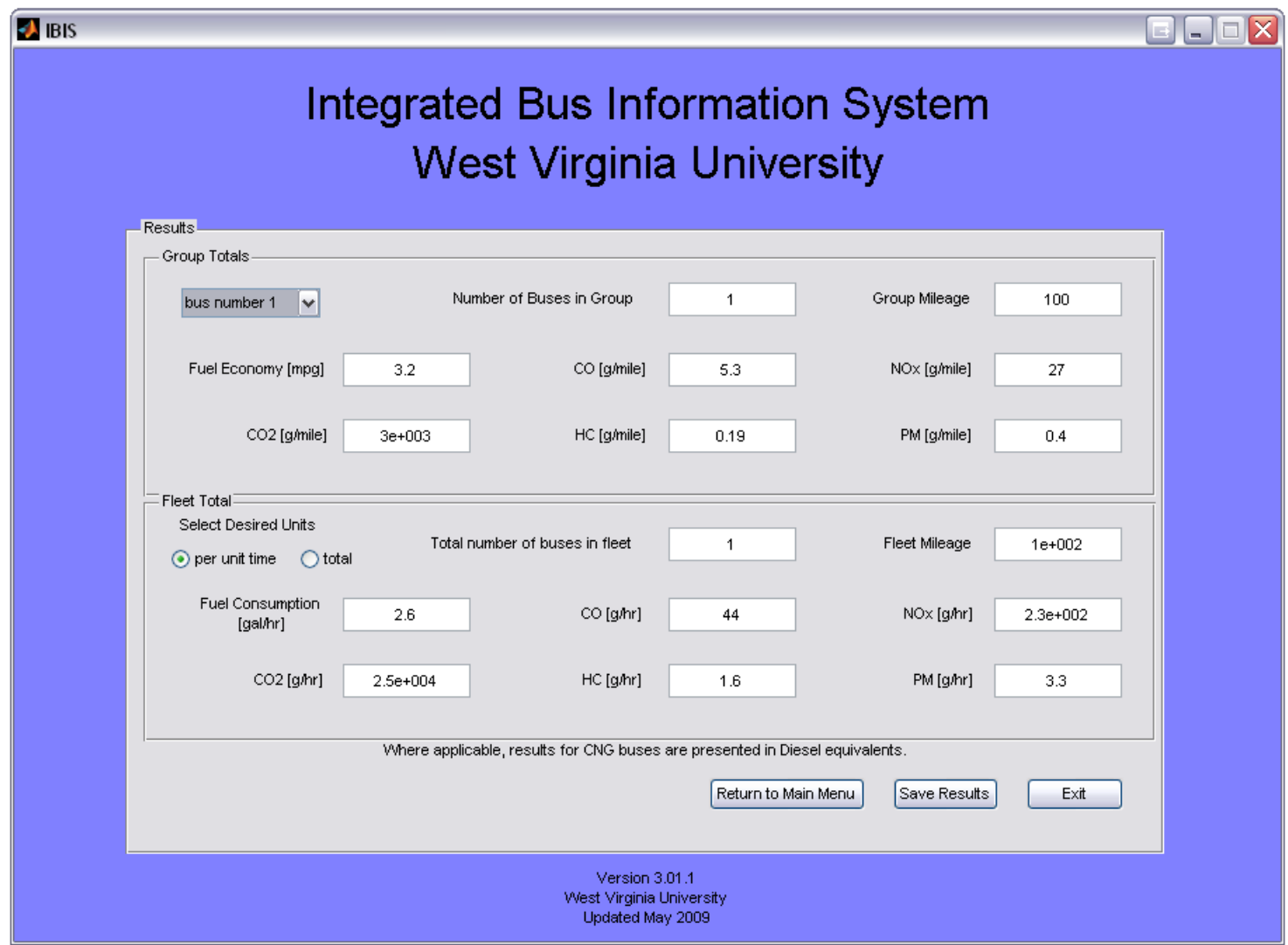

Figure 5.6: Results window for the offline IBIS tool 


\section{Chapter 6 Results}


The cycle generator was created to fill gaps in the available testing data. Without the additional cycles created by the cycle generator, the polynomial tool is unable to calculate reasonable models. As you can see in Figure 6.1, the search space has several gaps, such as between 25 and $42 \mathrm{mph}$. It can be assumed that interpolation will produce poor results in these areas.

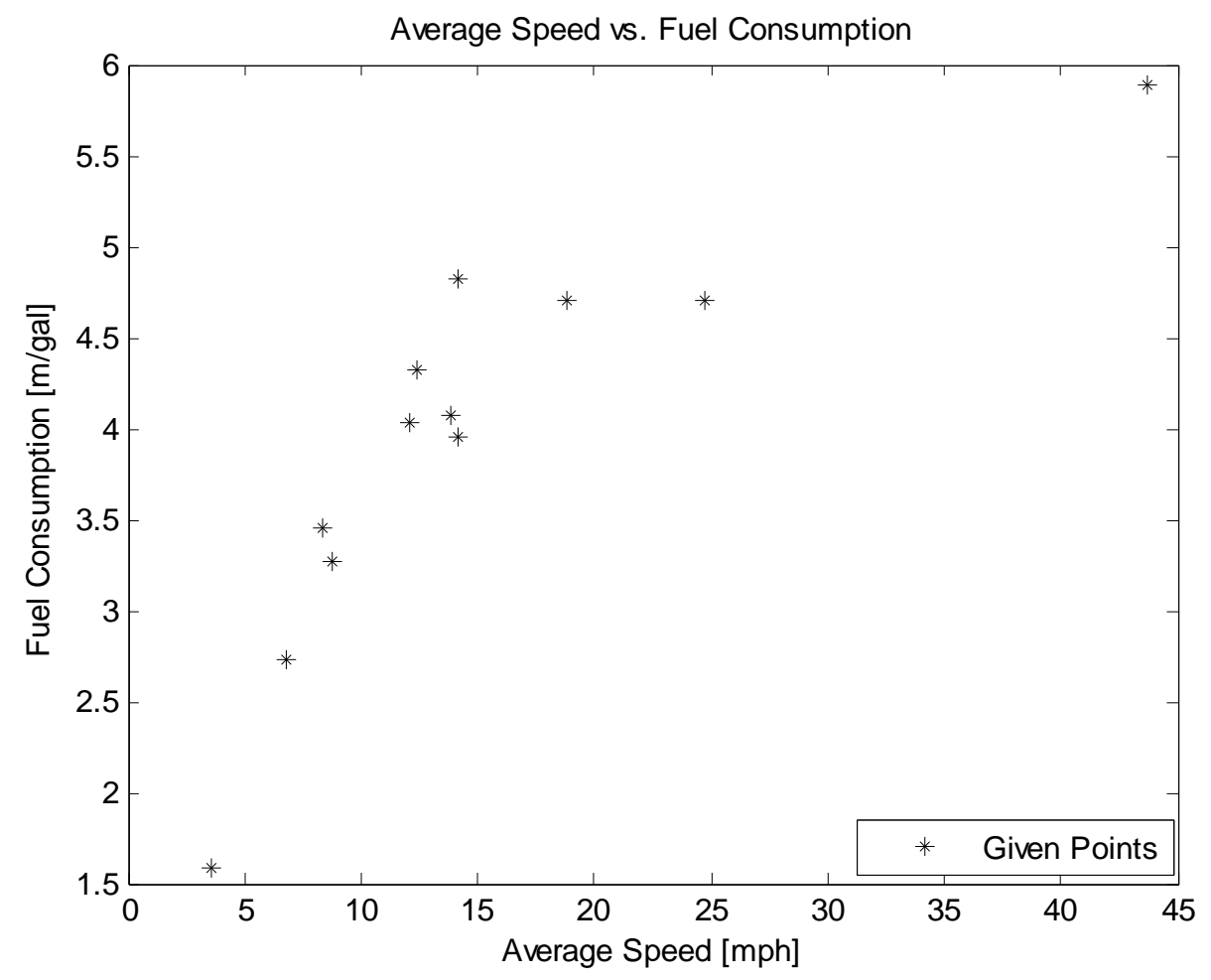

Figure 6.1: Average speed vs. fuel consumption illustrating gaps in the given (limited) data.

The cycle generator was used to create a database of new virtual driving cycles that would fill in the gaps in the data as shown above. Figure 6.2 shows this new data set. As you can see, the search space is more complete following cycle creation. The black points are the original 12 standard driving cycles, and the red points are the cycles that were generated with the driving cycle generator. 


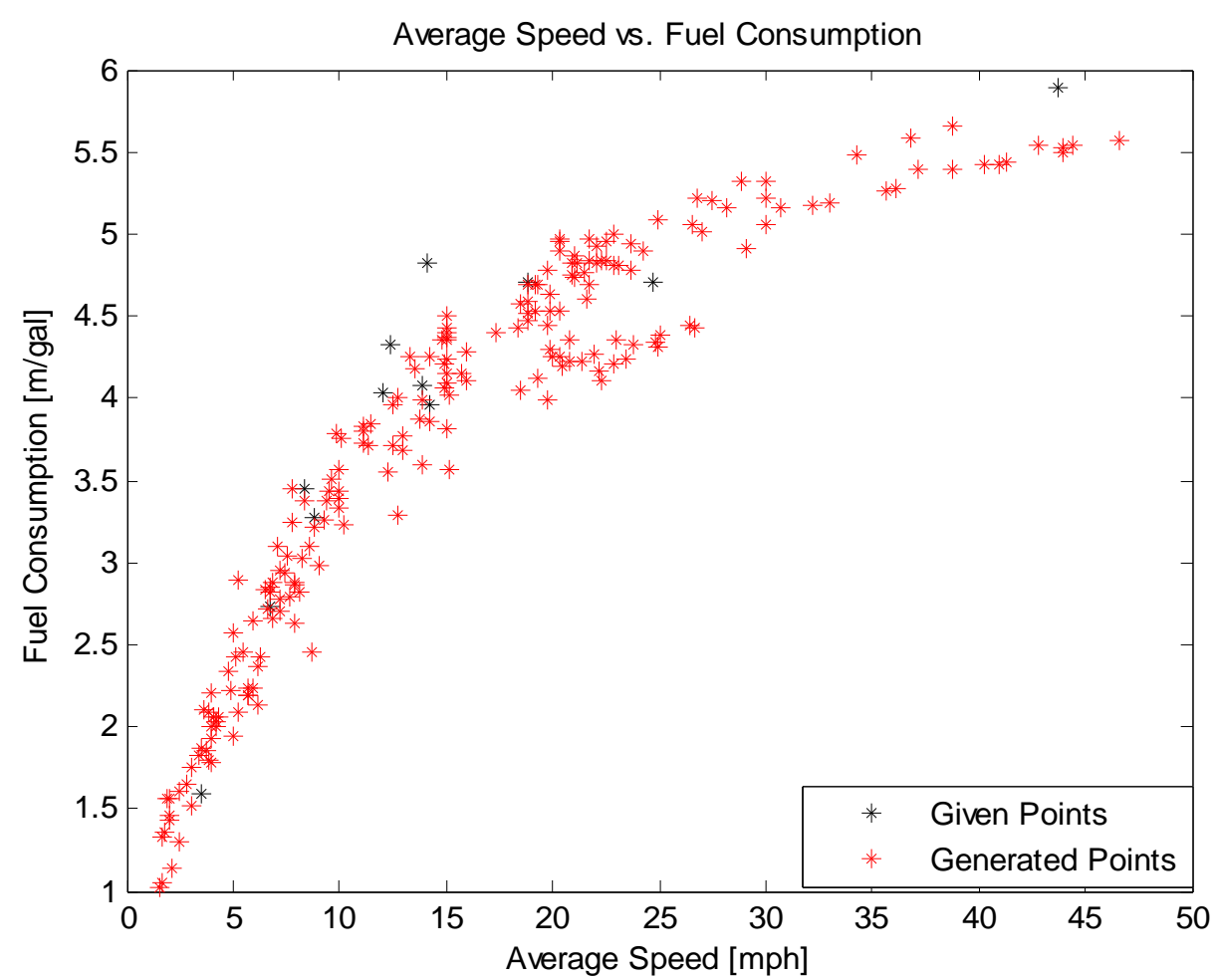

Figure 6.2: Expanded driving cycle database

Using the same example of a 2006 diesel bus from section 3.3, we see in Figure 6.3 that the PolyTool is unable to generate a reasonable model even after extensive manipulation of the regression terms. This is a $5^{\text {th }}$ degree model of fuel economy using average speed and number of stops per mile. 


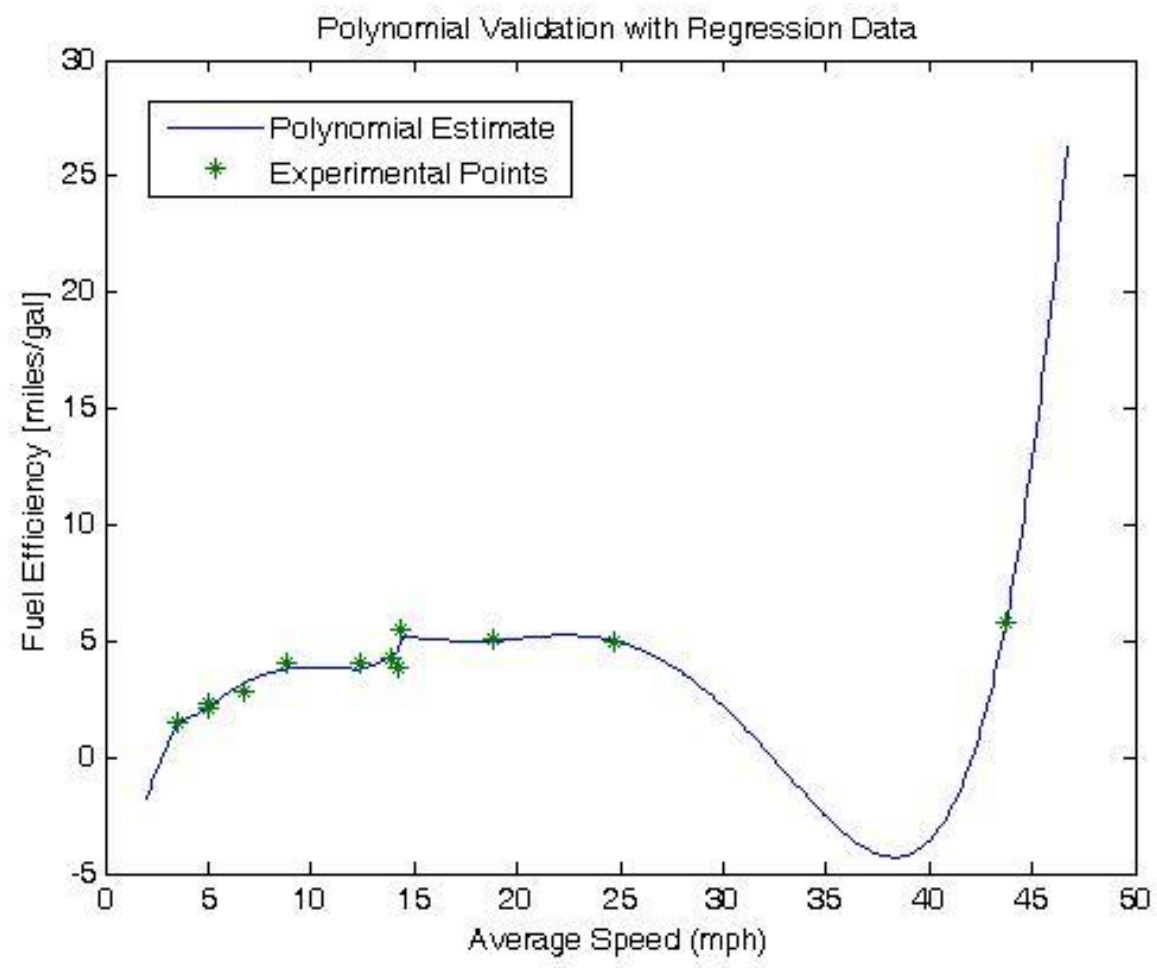

Figure 6.3: Projection of a model created with limited data

Not only is there undesirable behavior between points, but the model will return negative fuel economy values in certain areas. Comparing this limited model to that in Figure 6.4, it further demonstrates the effectiveness of the cycle generator to improve the polynomial model. 


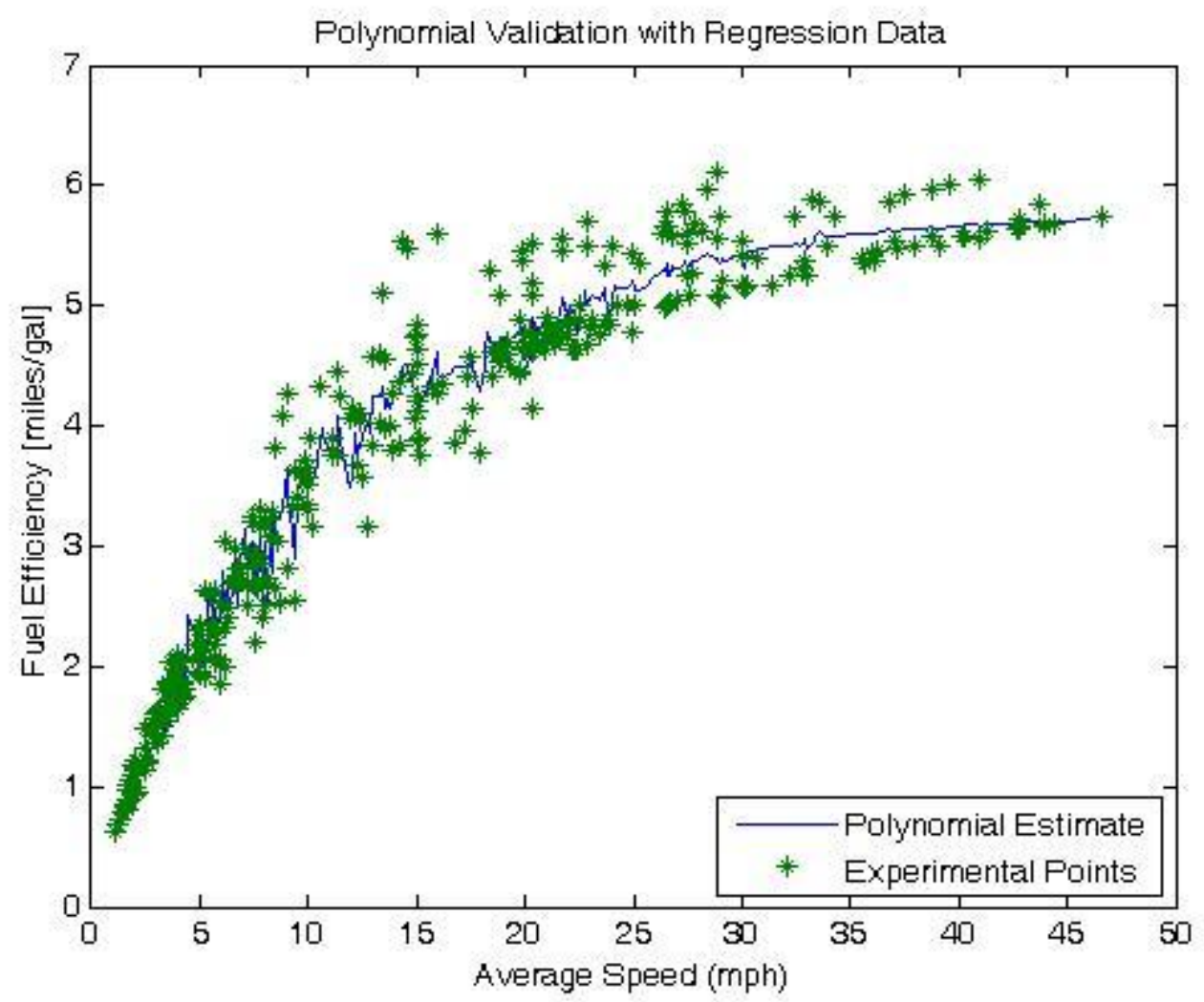

Figure 6.4: Projection of the model with an expanded cycle database

The overall goal of both the driving cycle generator and the PolyTool is to create backbone models for IBIS. To maintain usability, users should have the flexibility within IBIS to specify 1 to all 5 parameters to define their driving cycle since all 5 parameters may not be readily available. To meet this goal, the backbone models governing calculations in IBIS are constructed as pair of parameters. In this pair structure, average speed is considered to be mandatory and each pair is constructed accordingly.

Modeling, with an expanded cycle database, was implemented for fuel economy and each emission species for each back bone model. Figure 6.5 shows an example of a backbone model for a 2006 diesel transit bus. This backbone was used to compute fuel economy when average speed and percentage idle are given by the user; simarly, Figures 6.6-6.8 show example backbones for 2000 model year diesel, CNG, and diesel-hybrid respectively. Additional backbone examples for the 2006 diesel bus are available in Appendix C. 


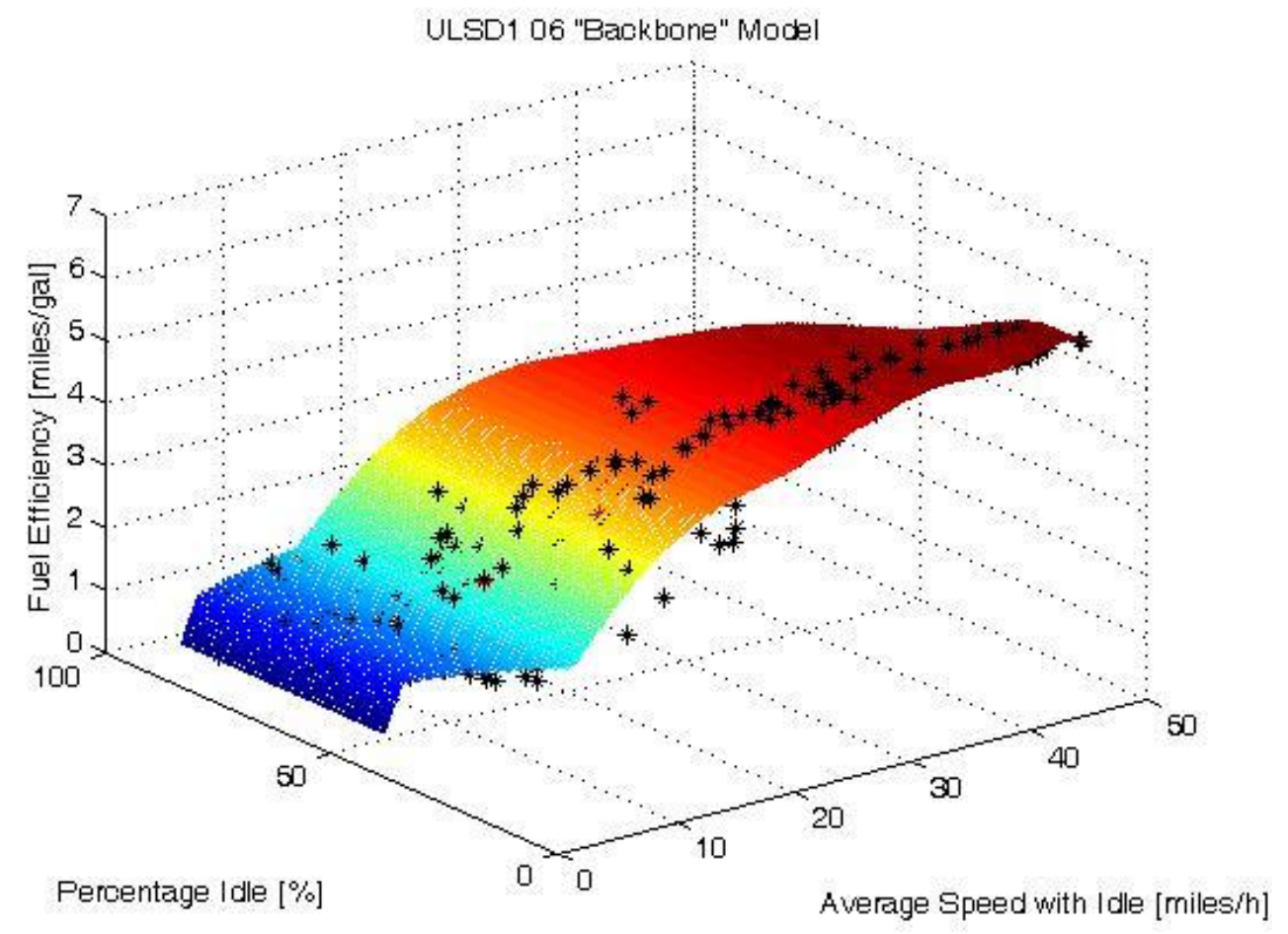

Figure 6.5: Example backbone model- 2006 Diesel Transit Bus

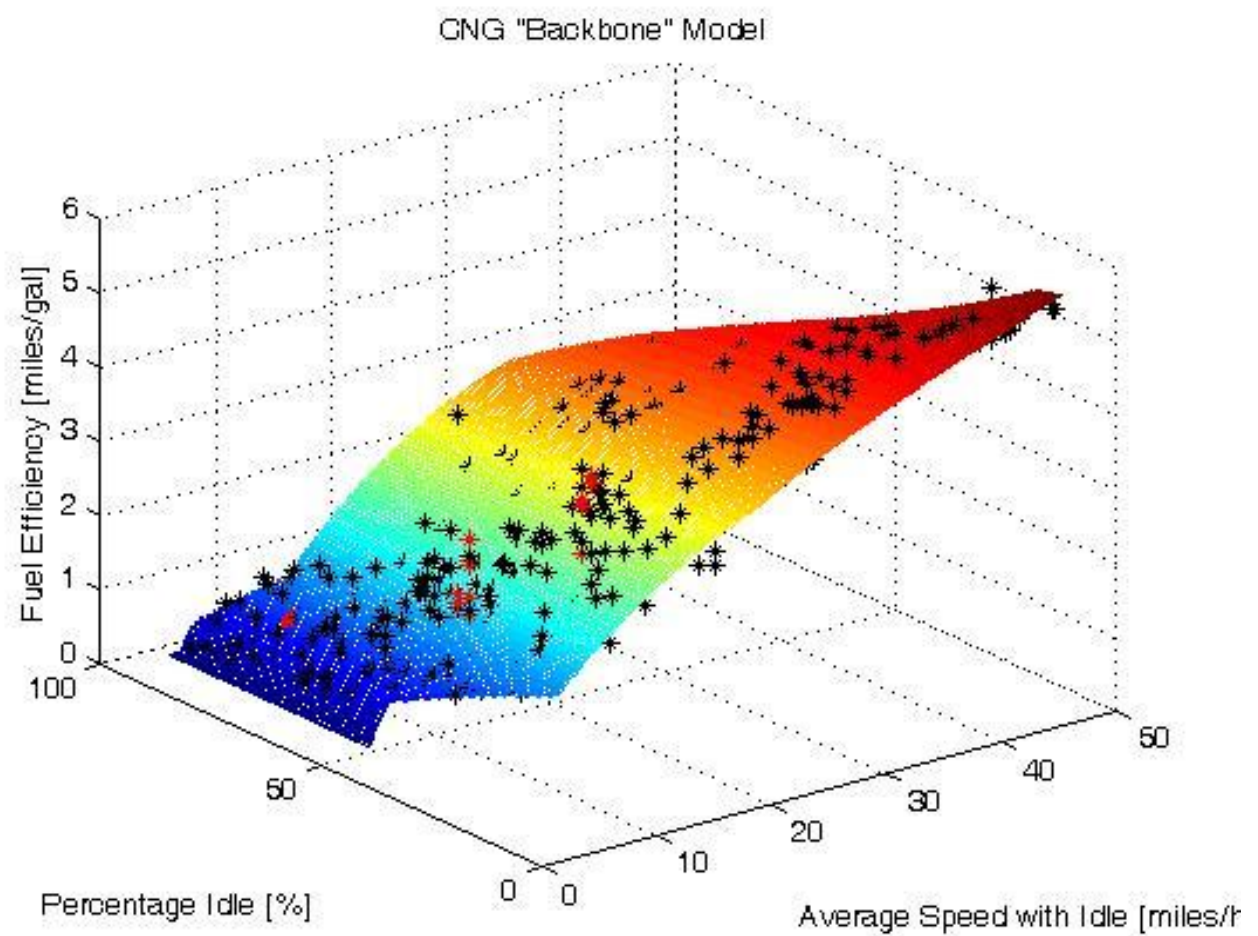

Figure 6.6: Example backbone model- 2006 Diesel Transit Bus 


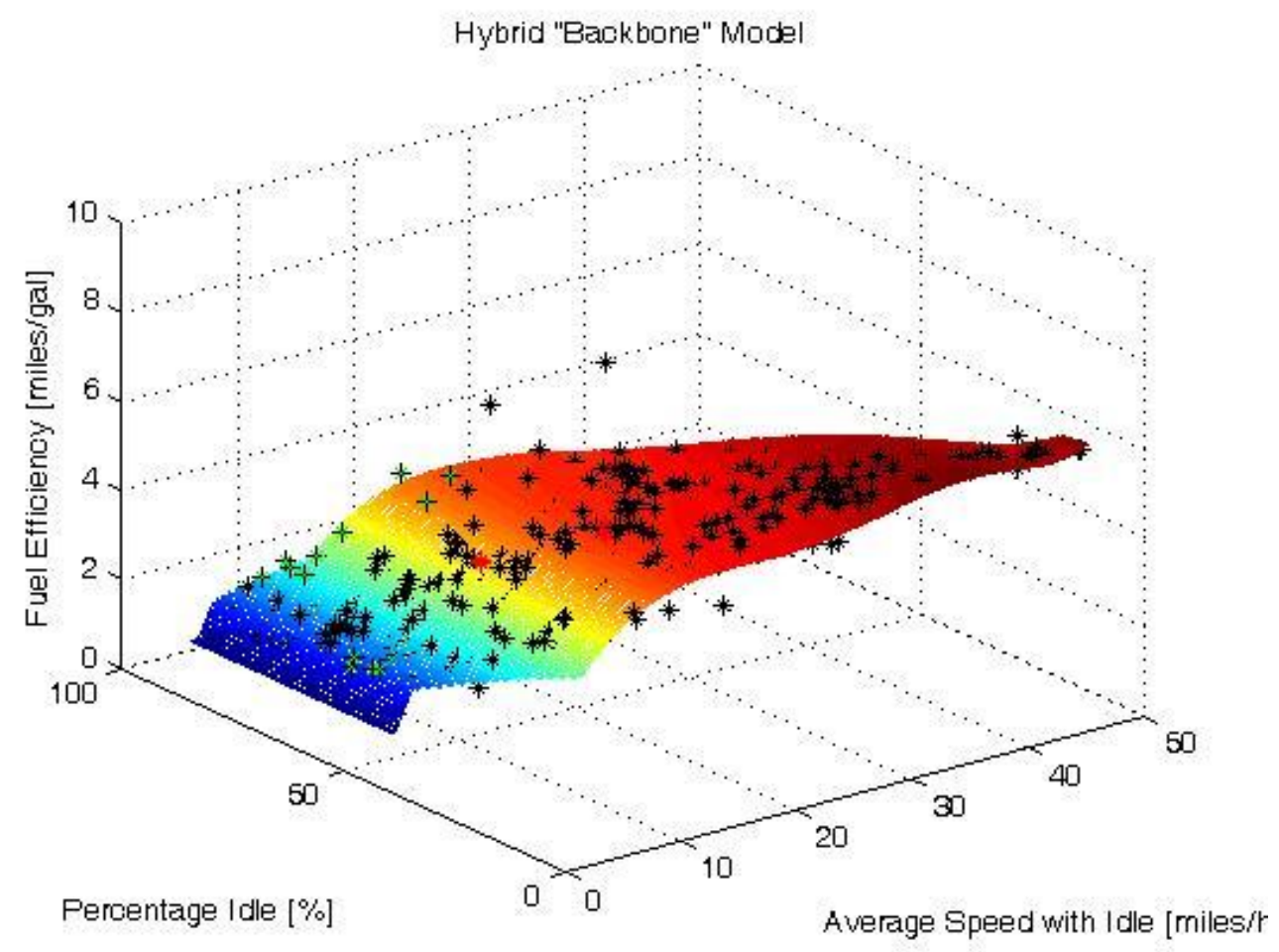

Figure 6.7: Example backbone model- 2006 Diesel Transit Bus

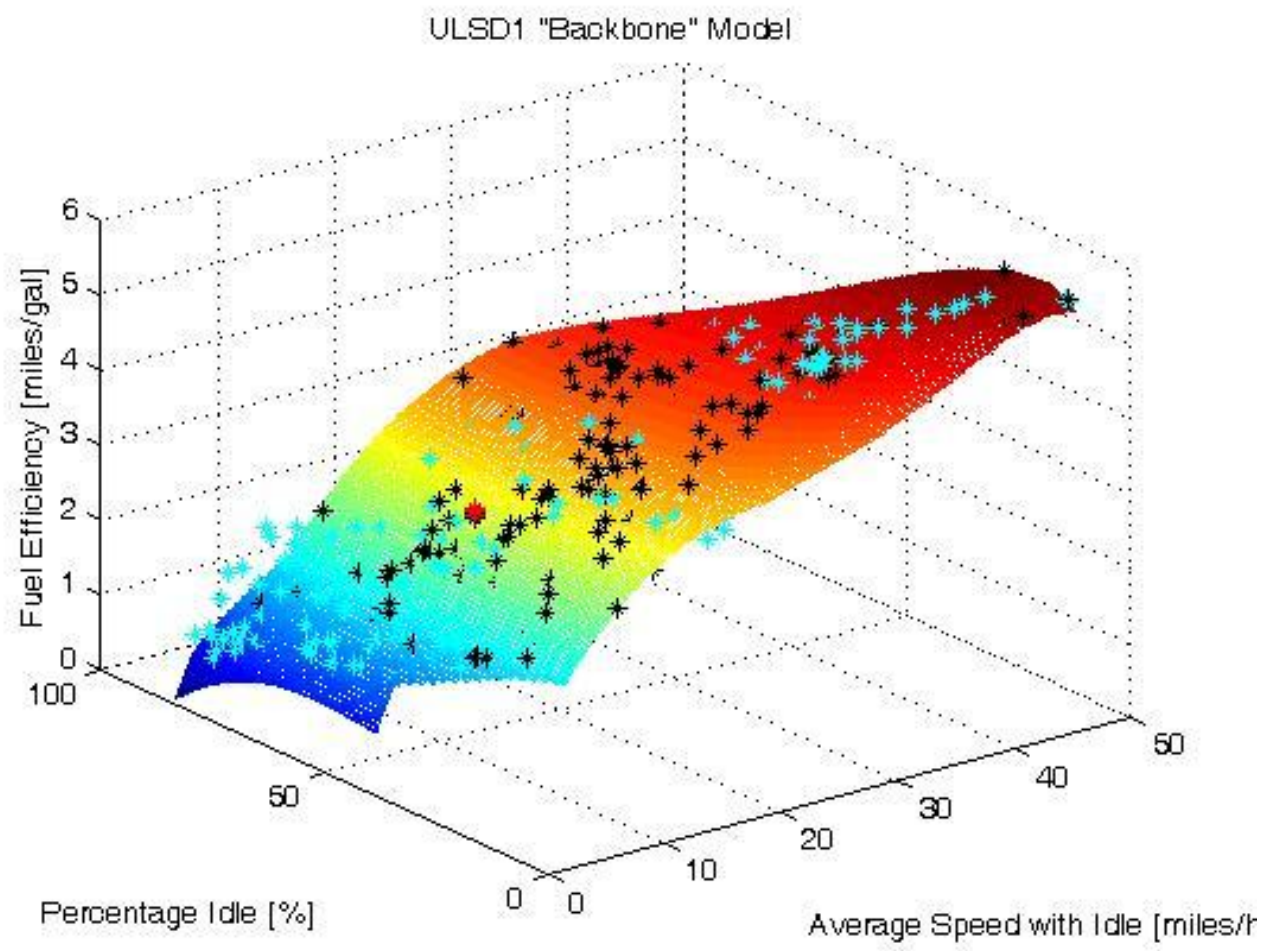

Figure 6.8: Example backbone model- 2006 Diesel Transit Bus 


\section{Chapter 7 Conclusions}


Development of IBIS required accurate models for engine fuel efficiency and emissions. To achieve this goal, advanced computational tools were developed to aid in the construction of backbone models for different fuel types, model years, and vehicle architectures. The first tool considered was a polynomial regression tool.

This tool is an effective method for creating multiple variable polynomial models. Being that the tool is structured in a manner that allows for extensive user manipulation of regression terms, the user is able to address the varying significance of secondary parameters in the output of the model. Although the tool is able to produce accurate and timely results, it is reliant upon a large dataset for proper modeling. Subsequent testing of the tool indicated that insufficient data was available to address this concern. This led to the necessary development of a driving cycle generator.

The driving cycle generator operates using a genetic search technique. It reorders microtrips assembled from existing test data. The cycle generator was able to produce new virtual driving schedules, complete with second-by-second emissions and fuel consumption data. These new cycles were created to fill gaps in the available test data search space. Not only did the addition of newly created cycles vastly improve the accuracy of model generated with the polynomial tool, but it also aided in the definition of acceptable limits for user inputs for the IBIS tool.

Upon integration and subsequent testing of these tools, backbone models were created for emissions and fuel consumption. These models were then introduced to the overall IBIS tool. The effectiveness of these tools can be realized by comparing IBIS output to available prediction software. The final IBIS product shows comparable results to available methodologies, but the usability and approachability of IBIS is superior to these available software packages.

While backbone models created by the polynomial regression tool are reliant upon large datasets, financial and time costs associated in retrieving this data are alleviated through the integrated use of the cycle generator. When combined, these two tools provide accurate models and insight to the domain of a vehicle operating at or beyond standard driving cycles. 


\section{References}

1. "Calculating emission inventories for vehicles in California User's Guide." California Air Resources Board. <http://www.arb.ca.gov/msei/onroad/downloads/docs/ user_guide_emfac2007.pdf $>$.

2. Rakha, Hesham, Kyoungho Ahn, and Antonio Trani. "Comparison of MOBILE5a, MOBILE6, VT-MICRO, and CMEM models for estimating hot-stabilized light-duty gasoline vehicle emissions." Canadian Journal of Civil Engineering 30.6 (Dec. 2003): 1010-1021.

3. "User's Guide to MOBILE6.1 and MOBILE6.2." United States Environmental Protection Agency. 2003 < http://www.epa.gov/OTAQ/models/mobile6/420r03010.pdf>.

4. Cumberworth, Mitch, et al. "MOVES-HVI Demonstration Version User Guide." United States Environmental Protection Agency. 2007 < http://www.epa.gov/otaq/models/ moves/420b09008.pdf>.

5. Rakha, Hesham, Kyoungho Ahn, and Antonio Trani. "Development of VT-Micro model for estimating hot stabilized light duty vehicle and truck emissions." Transportation Research: Part D 9.1 (Jan. 2004): 49.

6. Tu, Jun. "Investigation of Predictive Models for Emissions and Fuel Economy of Transit Buses (tentative).” Diss. West Virginia University, 2009. Morgantown, WV: in progress.

7. Klein, Vladislav, and Eugene A. Morelli. Aircraft System Identification Theory and Practice. Reston, VA: American Institute of Aeronautics and Astronautics, 2006.

8. Michalewicz, Z., Genetic Algorithms + data Structure = Evolution Programs. New York, NY: Springer Verlag, 1994.

9. Davis, L., Handbook of Genetic Algorithms. New York, NY: Van Nostrand Reinhold, 1991.

10. Min Li, et al. "Evolution of virulence in epidemic community-associated methicillinresistant Staphylococcus aureus." Proceedings of the National Academy of Sciences of the United States of America 106.14 (07 Apr. 2009): 5883-5888.

11. Chambers, Henry F. "The Changing Epidemiology of Staphylococcus Aureus?." Emerging Infectious Diseases 7.2 (Mar. 2001): 178.

12. Holland, J.H.. Adaptation in Natural and Artificial Systems: An Introductory Anaylsis with Applications to Biology, Control, and Artificial Intelligence. Cambridge, Mass: MIT Press, 1992. 
13. Hirani, H., and N.P. Suh.. "Journal bearing design using multiobjective genetic algorithm and axiomatic design approaches." Tribology International 38.5 (May 2005): 481491.

14. Kim, Cheol-Ung, et al. "Optimal design of filament wound type 3 tanks under internal pressure using a modified genetic algorithm." Composite Structures 71.1 (15 Oct. 2005): $16-25$.

15. Kers, J., and J. Majak.. "Modelling a new composite from a recycled GFRP." Mechanics of Composite Materials 44.6 (Nov. 2009): 623-632.

16. Guo, Jiangfeng, Mingtian Xu, and Lin Cheng. "The application of field synergy number in shell-and-tube heat exchanger optimization design." Applied Energy 86.10 (Oct. 2009): 2079-2087.

17. Sheng, W., et al. "Consensus fingerprint matching with genetically optimised approach." Pattern Recognition 42.7 (July 2009): 1399-1407.

18. Cheng, M.-Y., and C.-S. Lin.. "Genetic algorithm for control design of biped locomotion." Journal of Robotic Systems 14.5 (May 1997): 365-373.

19. Perhinschi M. G., "Controller Design for Autonomous Helicopter Model Using a Genetic Algorithm", Proceedings of the American Helicopter Society 53rd Annual Forum, Virginia Beach VA, USA, May 1997, pp. 788-794.

20. Crawford L. S., Cheng V. H. L., Menon P. K., "Synthesis of Flight Vehicle Guidance and Control Laws Using Genetic Search Methods", AIAA99-4153 Proceedings of the AIAA Guidance, Navigation, and Control Conference, August 9-11, 1999 Portland, OR.

21. Sweriduk G. D., Menon P. K., Steinberg M. L., "Robust Command Augmentation System Design Using Genetic Search Methods", AIAA98-4131, Proceedings of the AIAA Guidance, Navigation, and Control Conference, August, 1998 Boston, MA.

22. Perhinschi M. G., "Parameter Optimization Via Genetic Algorithm of Fuzzy Controller for Autonomous Airvehicle", Proceedings of the AIAA Guidance, Navigation, and Control Conference, Portland OR, USA, August 1999, pp. 790-797.

23. Krishnakumar K., Goldberg D. E., "Control System Optimization Using Genetic Algorithms," Journal of Guidance, Control and Dynamics, Number 3, 1992.

24. Ljung, Lennart. System Identification: Theory for the User. Englewood Cliffs: PrenticeHall, 1987. 


\section{Appendix A- PolyTool User Guide}

\section{Requirements}

The Polynomial tool is created in MATLAB ver. 7.4.0. While MATLAB is generally backwards compatible, GUI files contained herein may not function properly in other versions of MATLAB.

\section{Introduction}

PolyTool allows a user to perform polynomial regression for 12 standard driving cycles, as well as points created with the GA tool. This regression is flexible in that a user may manipulate regression terms in order to satisfy a more realistic "real world" fit to the data. More specifically, in order to address secondary parameters and their affect on the more important primary parameter.

\section{Using the PolyTool}

First a user must load MATLAB. Next change your current directory to the appropriate directory. In the command window type "PolyGUI." You will be presented with the following window (A.1).

Figure A.1: Initialize Window

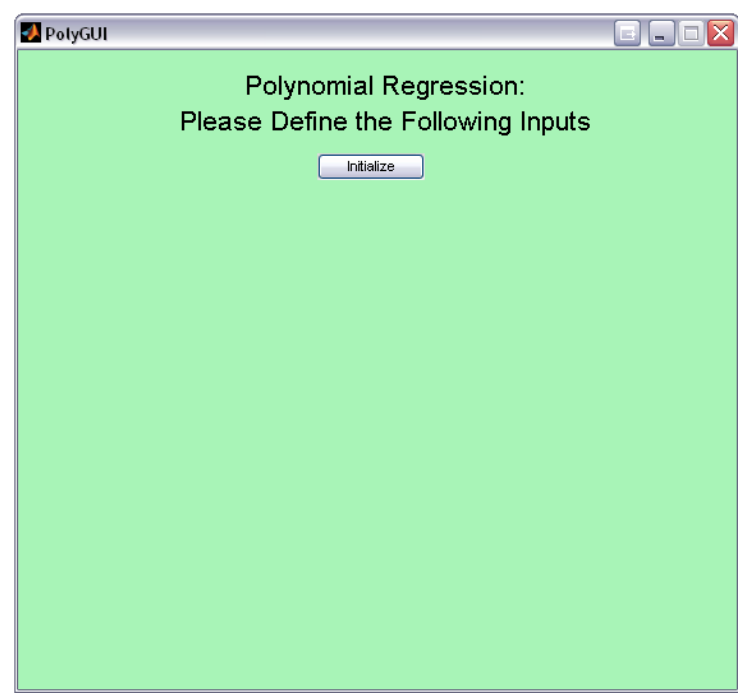

Next click initialize to start the tool. The user is now presented with a new window (A.2) in which to select output parameters defining the polynomial-shown below. In this version of the PolyTool, the user is limited to selecting Average Speed with Idle, Percentage Idle, Stops per Mile, Standard Deviation of Speed with Idle, and Kinetic Intensity. Input parameters are selected simply by clicking the radio button next to the appropriate input. The user must also 
specify degree of the polynomial. 1 is a linear fit, 2 is a second order fit, etc. For most practical purposes, polynomials of a much higher order yield little to no additional benefit over polynomial of 6 or 7 degrees. This leads to overfitting of the dataset and is not indicative of a "real world" solution. Finally the user must select an output for the polynomial. This can be one of the following: Fuel Consumption [gal/mile], $\mathrm{CO}$ [g/mile], $\mathrm{CO}_{2}[\mathrm{~g} / \mathrm{mile}], \mathrm{HC}[\mathrm{g} / \mathrm{mile}], \mathrm{NOx}$ [g/mile], or PM [g/mile]. It should be noted that this point PM data is largely unreliable due to the lack of accurate second-by-second PM emissions data. PM will yield a polynomial; however, regression is preformed only on the 12 standard driving cycles.

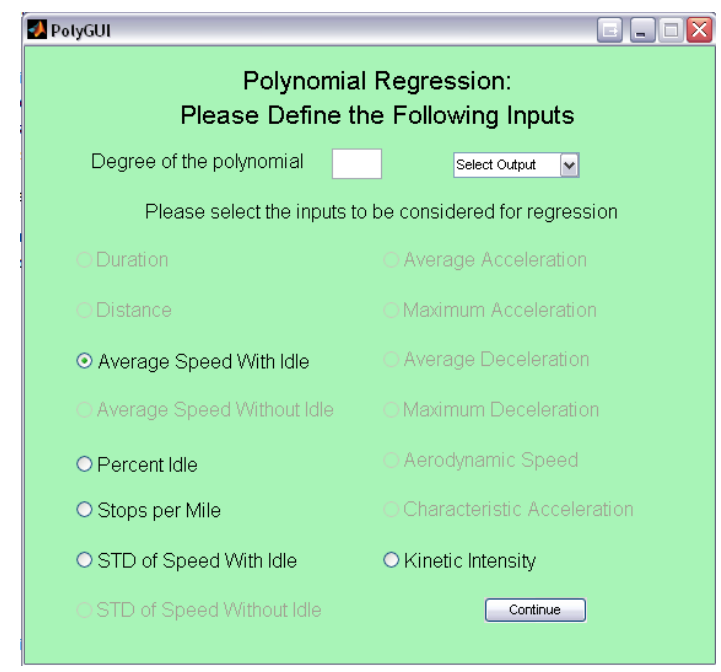

Figure A.2: Input Window

Once all input parameters have been defined, click continue and you will be presented with the next window-shown below (A.3). Again click initialize to load the menu. Now the user must select which driving cycles are to be used in the regression. Standard cycles as well as cycles created by the GA tool may be selected. These standard cycles include: ART, BEELINE, BRAUN, CBD, COMM, CSHVR, Manhattan, NYBUS, NY Composite, OCTA, UDDS, and/or, WMATA. To select a driving cycle for regression simply click the check box next to its name. If you would like to consider generated cycles from the GA tool (highly encouraged) click the check box next to "Include Additional Cycles" 


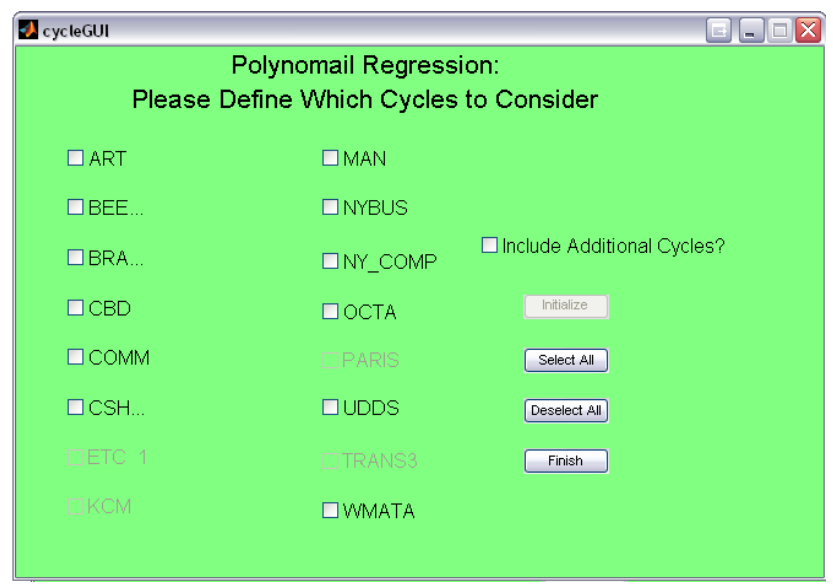

Figure A.3: Cycle Selection Window

Once all selections are made, click finish to run the tool.

After the tool has computed the regression for the specified inputs the user will be presented with a set of results windows (A.4-5). Of particular note is the window which shows a 2 dimensional projection of the polynomial- Average Speed vs. Emission/Fuel Consumption output- as seen below. Due to the projection it may appear that the model is not continuous. This is a result of variation within the secondary parameter.

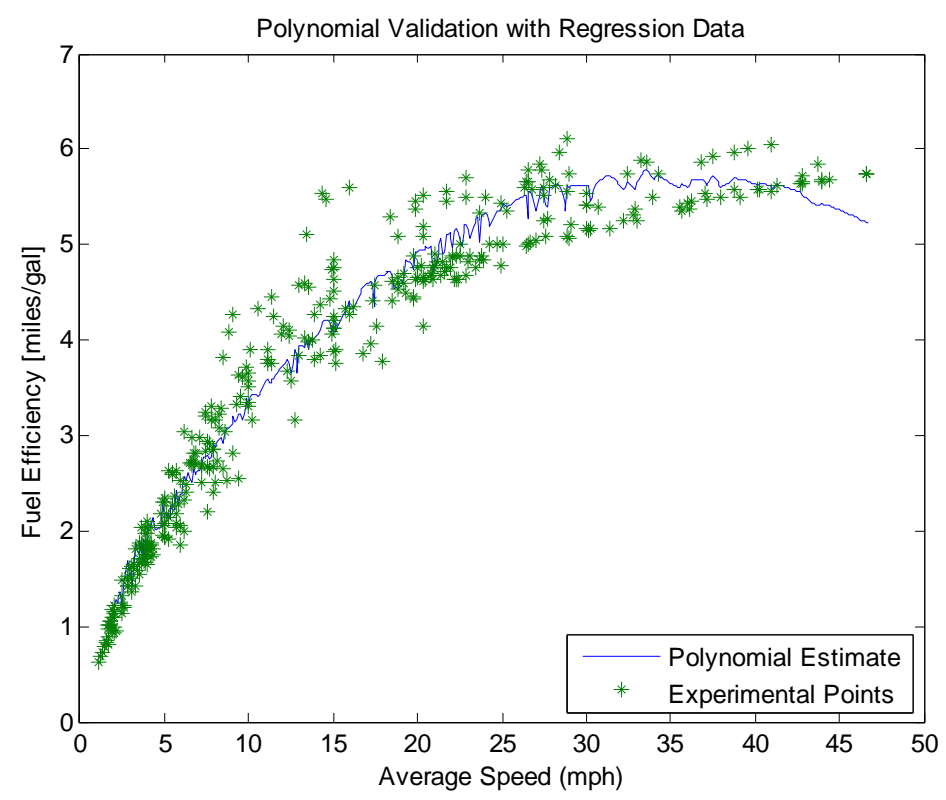

Figure A.4: Polynomial Projection 
Additional plots are presented as to ascertain the effect of varying the second parameter on the polynomial. These plots fix the secondary parameter, and identify points where the secondary parameter is within $20 \%$ of the fixed value. An example of this output window is shown below.

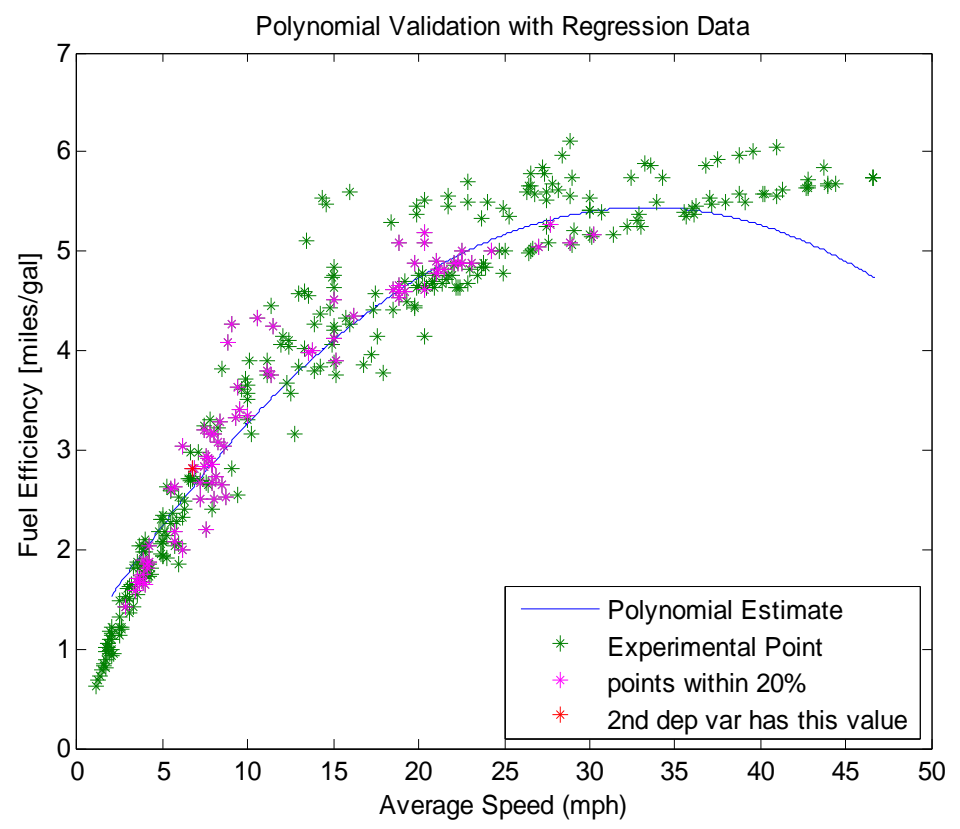

Figure A.5: Effect of Varying Second Parameter

The final window supplied by the tool is where the user may manipulate regression terms by eliminating thetas from the regression algorithm. These terms are presented as well as are their respective values. To remove a term, type its index in the text box within the window and click OK. The index is the order in which it appears on the screen. For example, the index of the term theta $_{0}$ in the example window below would be 1 since theta $a_{0}$ or the free term appears first in the list of thetas. Should you not wish to remove regression terms, simply click ok leaving the text box blank. Additionally, you may reset the list of regression terms by clicking reset. This essentially recomputes the polynomial using the original inputs supplied by the user, eliminating the need to reenter the inputs in case of mistakenly deleting a desired regression term. 


\section{Appendix B- Cycle Generator User Guide}

\section{Using the GA Tool}

\section{Requirements}

The Cycle Generator tool is created in MATLAB ver. 7.4.0. While MATLAB is generally backwards compatible, GUI files contained herein may not function properly in other versions of MATLAB.

\section{Introduction}

The Cycle Generator serves primarily to expand the 12 standard driving cycles employed by the PolyTool. Additionally, this tool illustrates practical limits for user inputs. For example, average speed without idle is defined as a primary parameter and limits the acceptable ranges of each secondary parameter. Reason dictates that a cycle having an average speed of $30 \mathrm{mph}$ would be unlikely to have a large number of stops per mile-i.e. 50 .

\section{Using the Cycle Generator}

First a user must load MATLAB. Next change your current directory to the appropriate directory. In the command window type "CycleGenerator02." You will be presented with the following window (B.1).

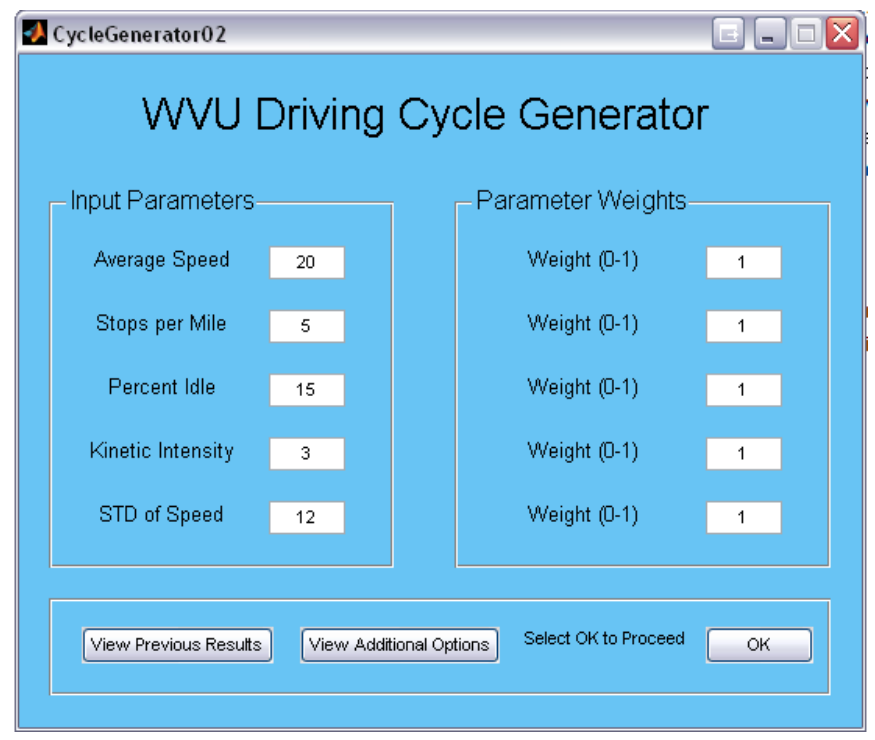

Figure B.1: Menu to Define Desired Output

In this window the user will specific your desired output parameters defining the newly created cycle. These parameters are average speed with idle, number of stops per mile, 
percentage idle, kinetic intensity, and standard deviation of speed with idle. Additionally the user may choose to specify the importance of each parameter in the final cycle. This is done by varying the weights associated with each parameter. These values are set to 1 by default indicating each parameter having equal importance. The user may also opt to change default parameters of the algorithm by clicking the View Additional Options button. Should the user wish to alter the default parameters they are presented with the following menu (B.2).

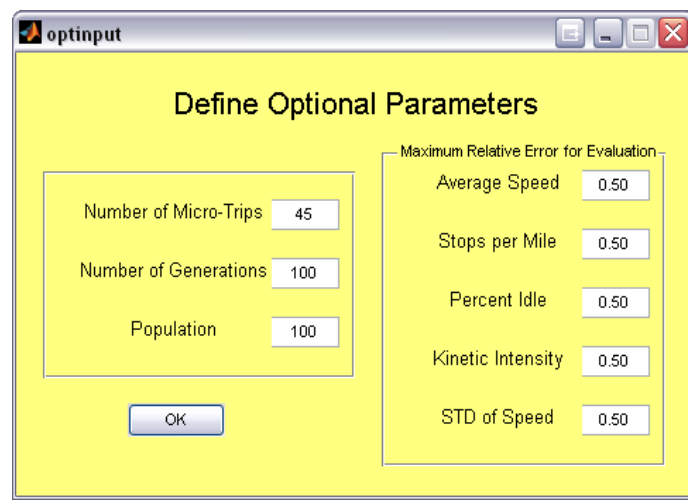

Figure B.2: Optional Parameters

In this menu, the user can change the length of each cycle (number of microtrips per cycle), number of generations (iterations), and the size of the initial population (number of cycles). The user may also change the maximum relative error for evaluation for each input parameter. Once all alterations have been made, click $O K$ and you will return to the main menu. Again click $O K$ to run the algorithm. Alternatively you may view the results from the last run of the algorithm by clicking View Previous Results; this option is available as long as a run has been completed previously regardless of whether or not the program has been closed.

Following a successful run of the tool the user is presented with the following results pane (B.3). 


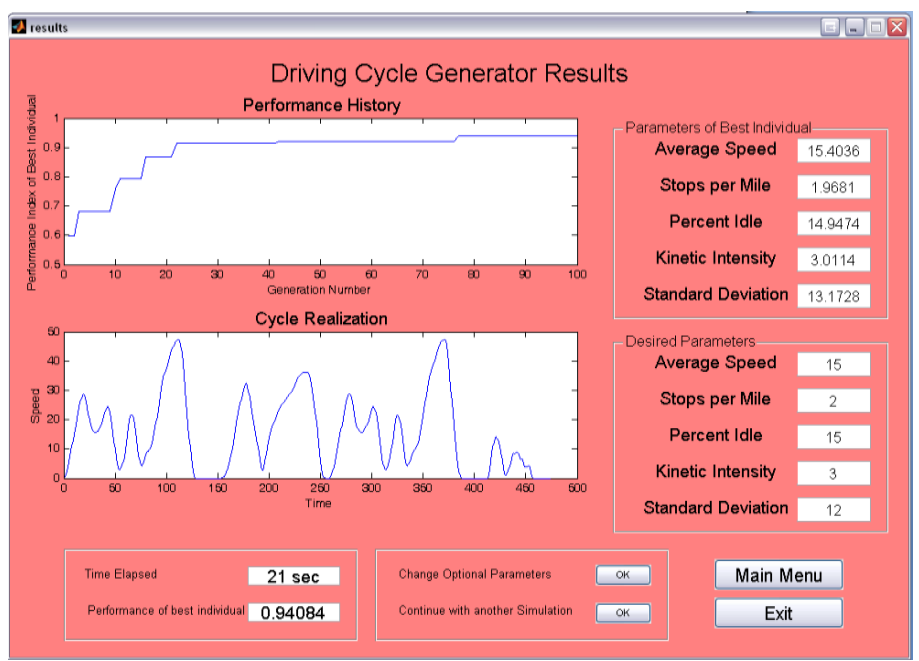

Figure B.3: Results Window

Here the user is presented with various data pertaining to the newly created cycle. Displayed is a time trace of the cycle, and a performance history of the cycle; also the time elapsed and the overall performance of the new cycle. The target parameters as well as the parameters gained by the tool are displayed.

The user may now opt to return to the main menu to create another new cycle. Alternatively the user can choose to continue the simulation with the same algorithm parameters (population number, cycle length, etc.). The user can also change the optional algorithm parameters and continue the simulation-i.e. the first simulation is run for 25 generation but only an additional 10 generations is desired. This method is useful when a user lacks the processing power to handle large numbers of generations. When all desired simulations have been completed, click exit to close the tool. 


\section{Appendix C- Created Backbone Models}

The following Figures show backbone models created for a 2006 model year ULSD1 transit bus.

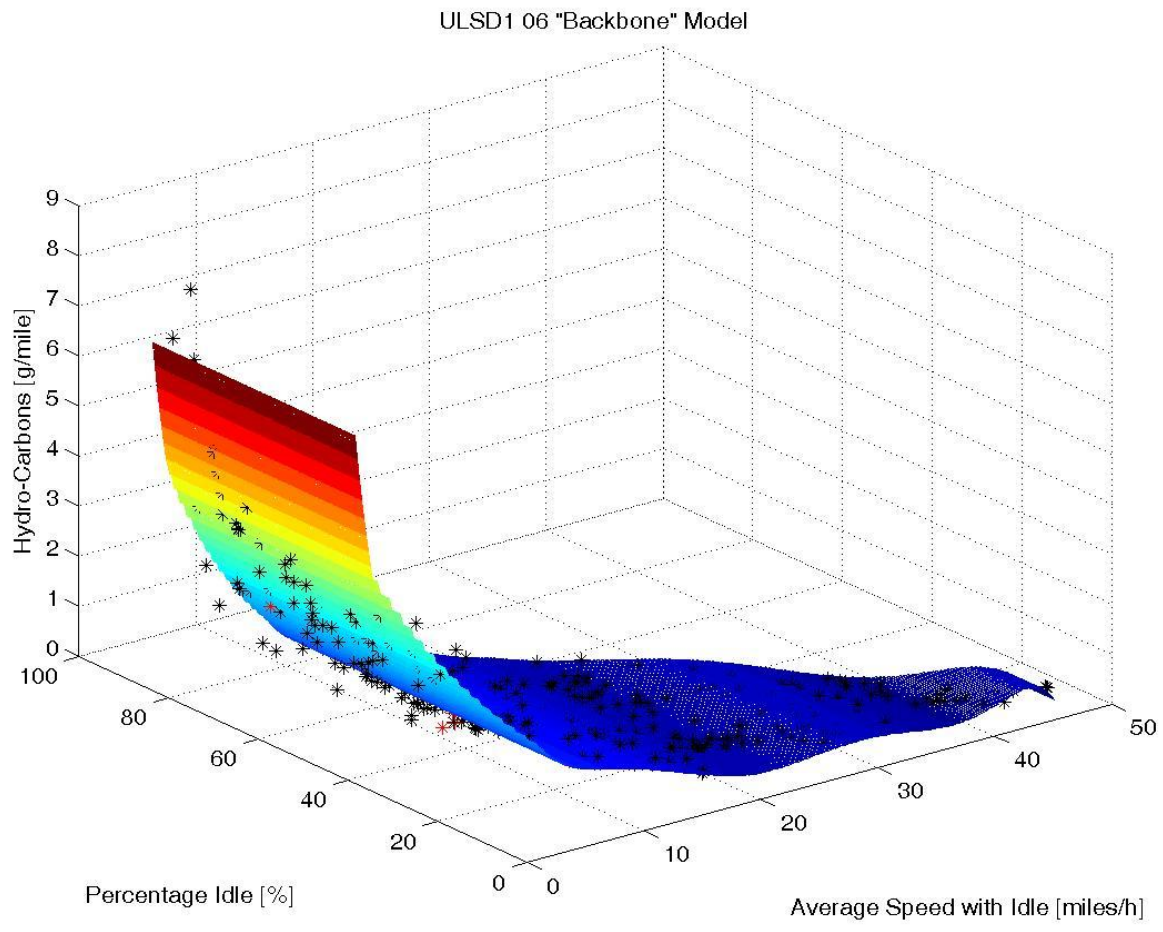

Figure C.1: Average speed \& PI backbone for HC 


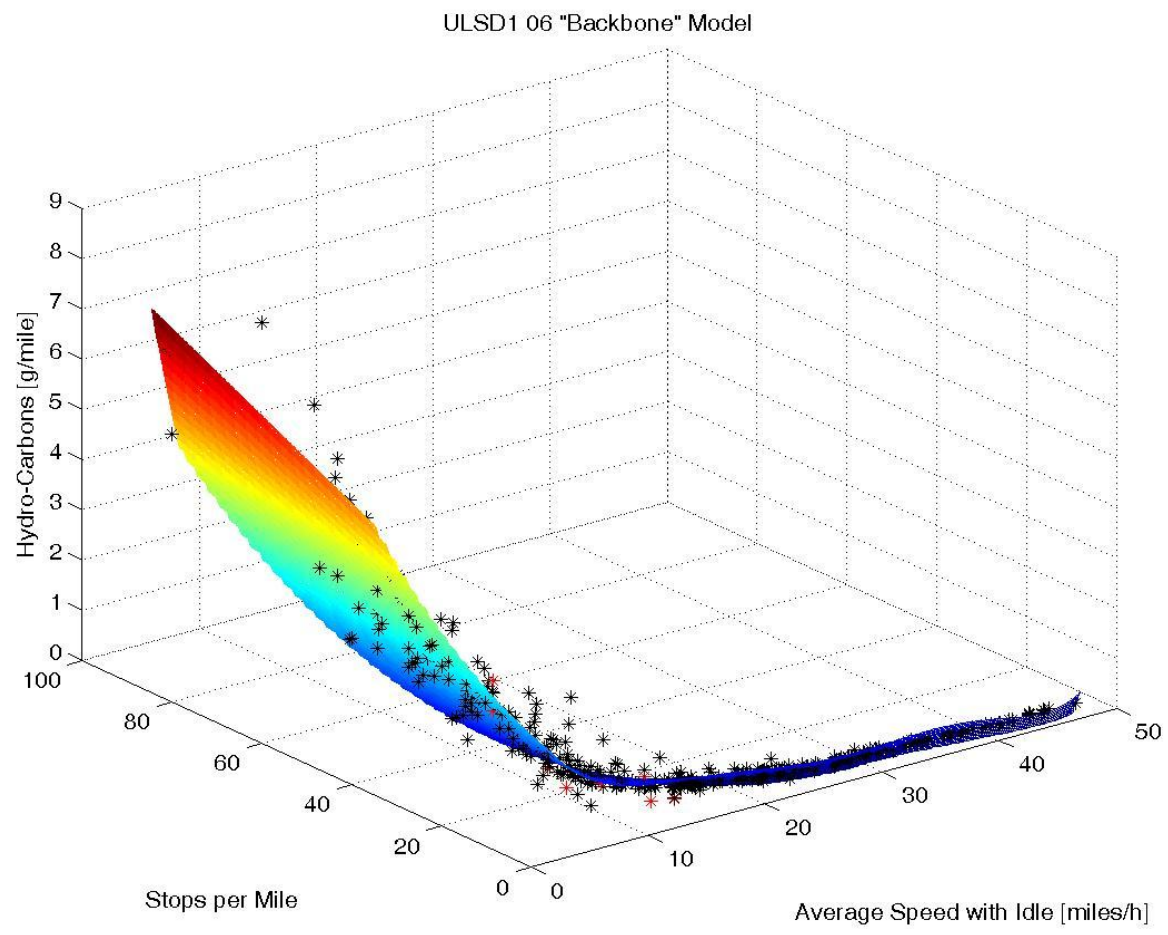

Figure C.2: Average speed \& stops per mile backbone for $\mathrm{HC}$

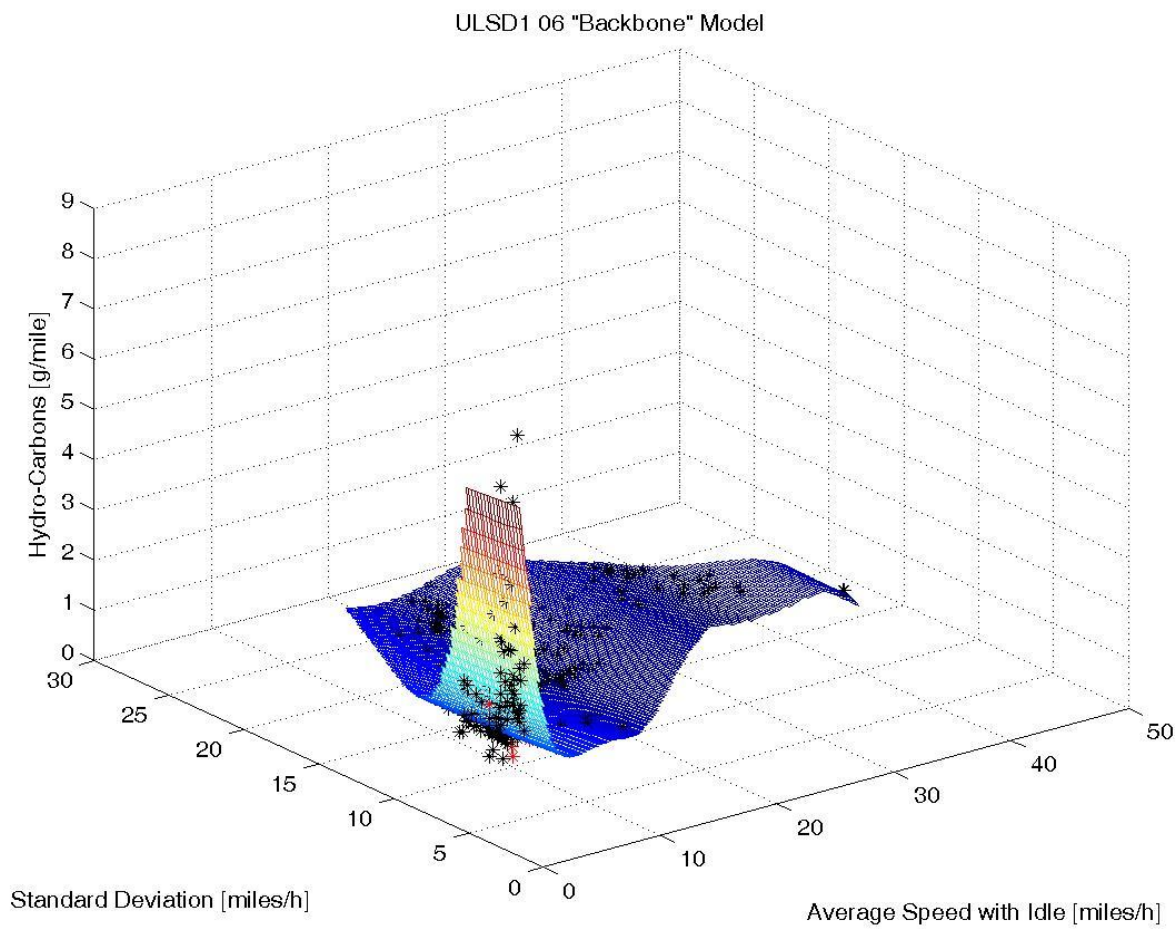

Figure C.3: Average speed \& STD backbone for HC 


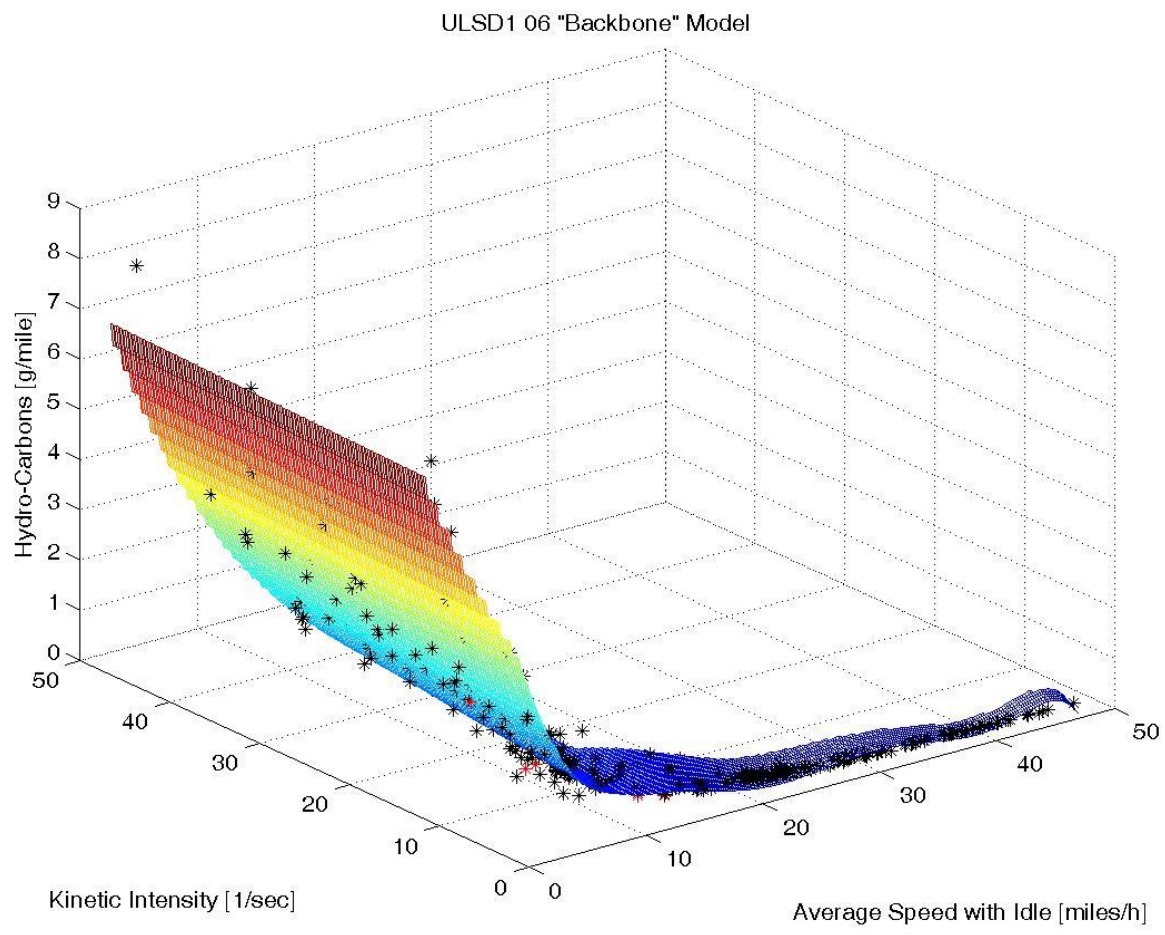

Figure C.4: Average speed \& KI backbone for HC

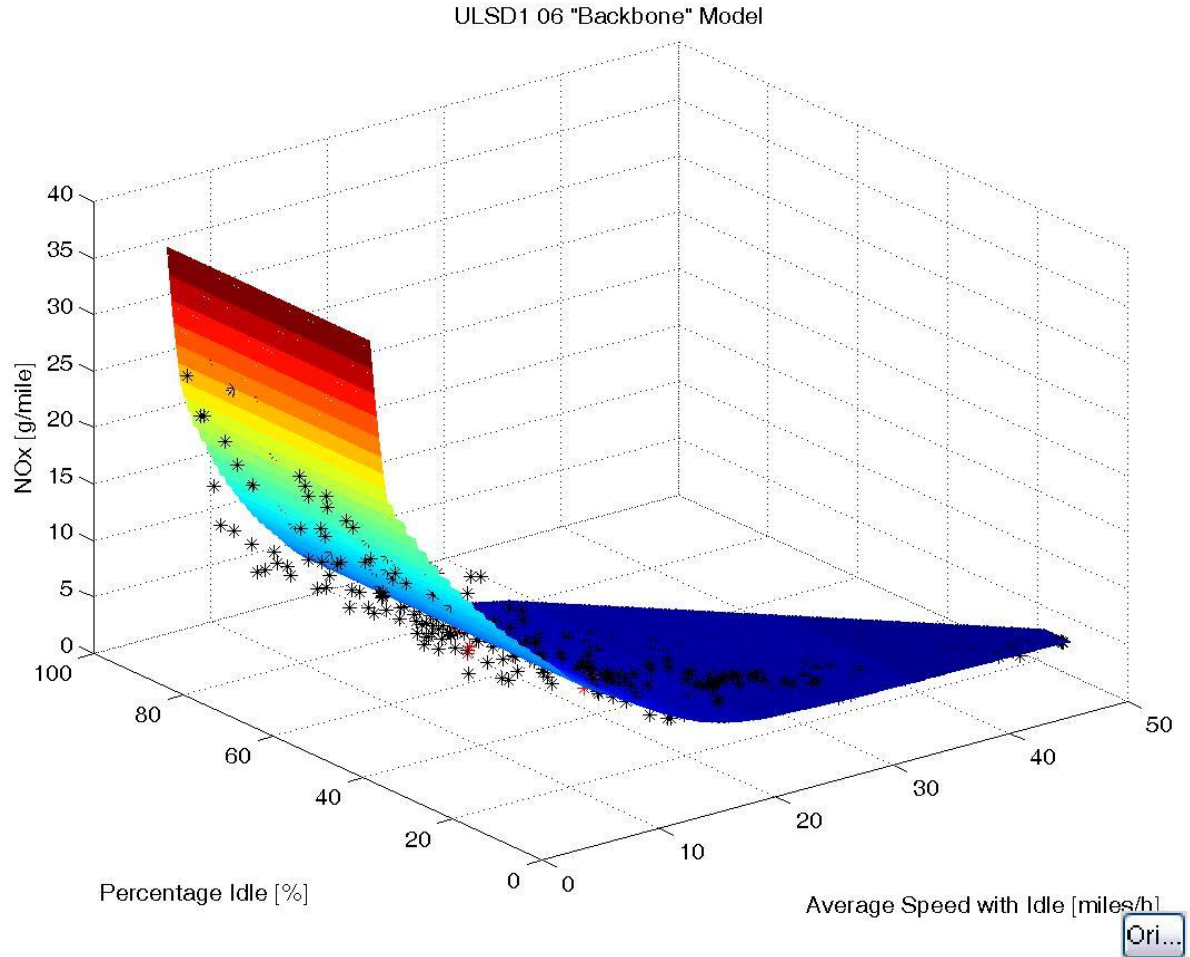

Figure C.5: Average speed \& PI backbone for NOx 


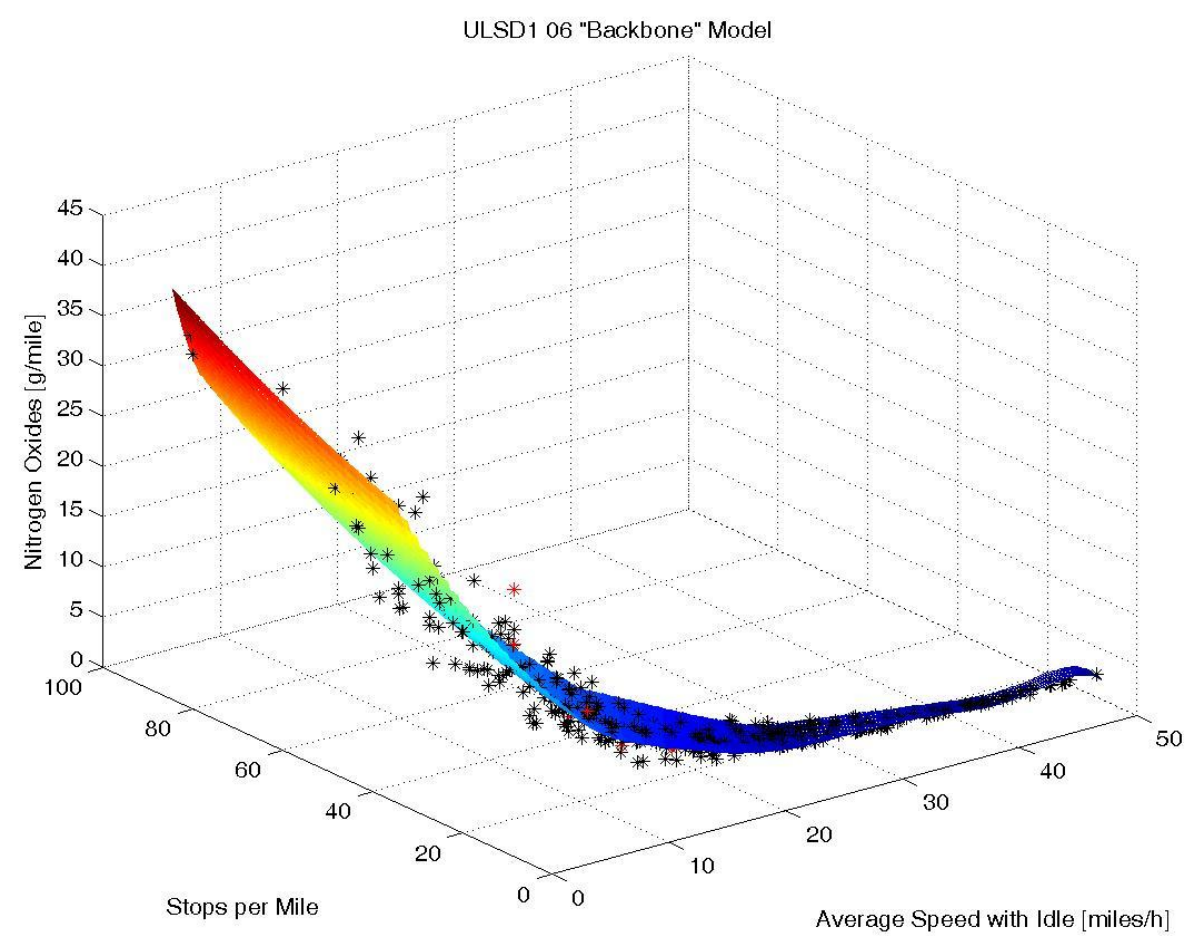

Figure C.6: Average speed \& stops per mile backbone for NOx

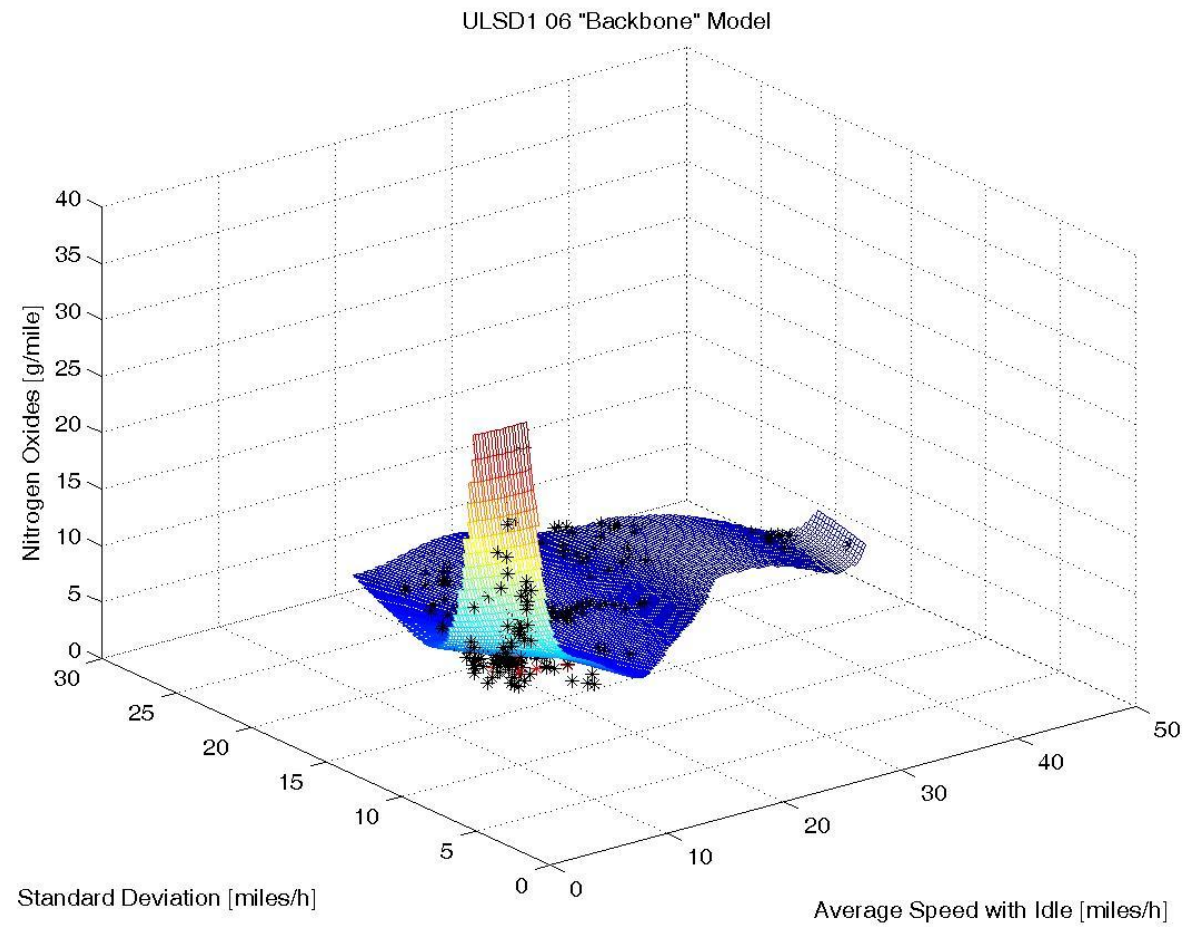

Figure C.7: Average speed \& STD backbone for NOx 


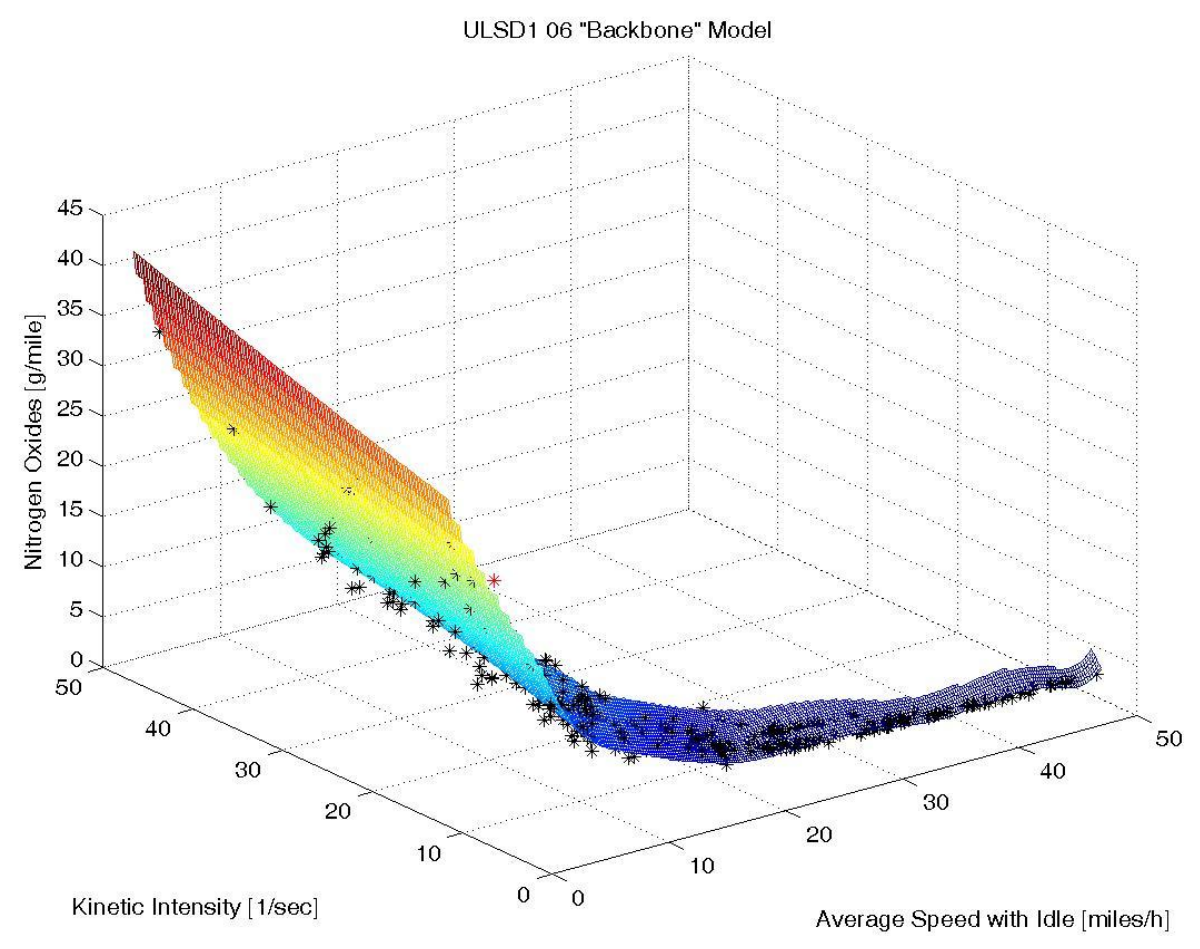

Figure C.8: Average speed \& KI backbone for NOx

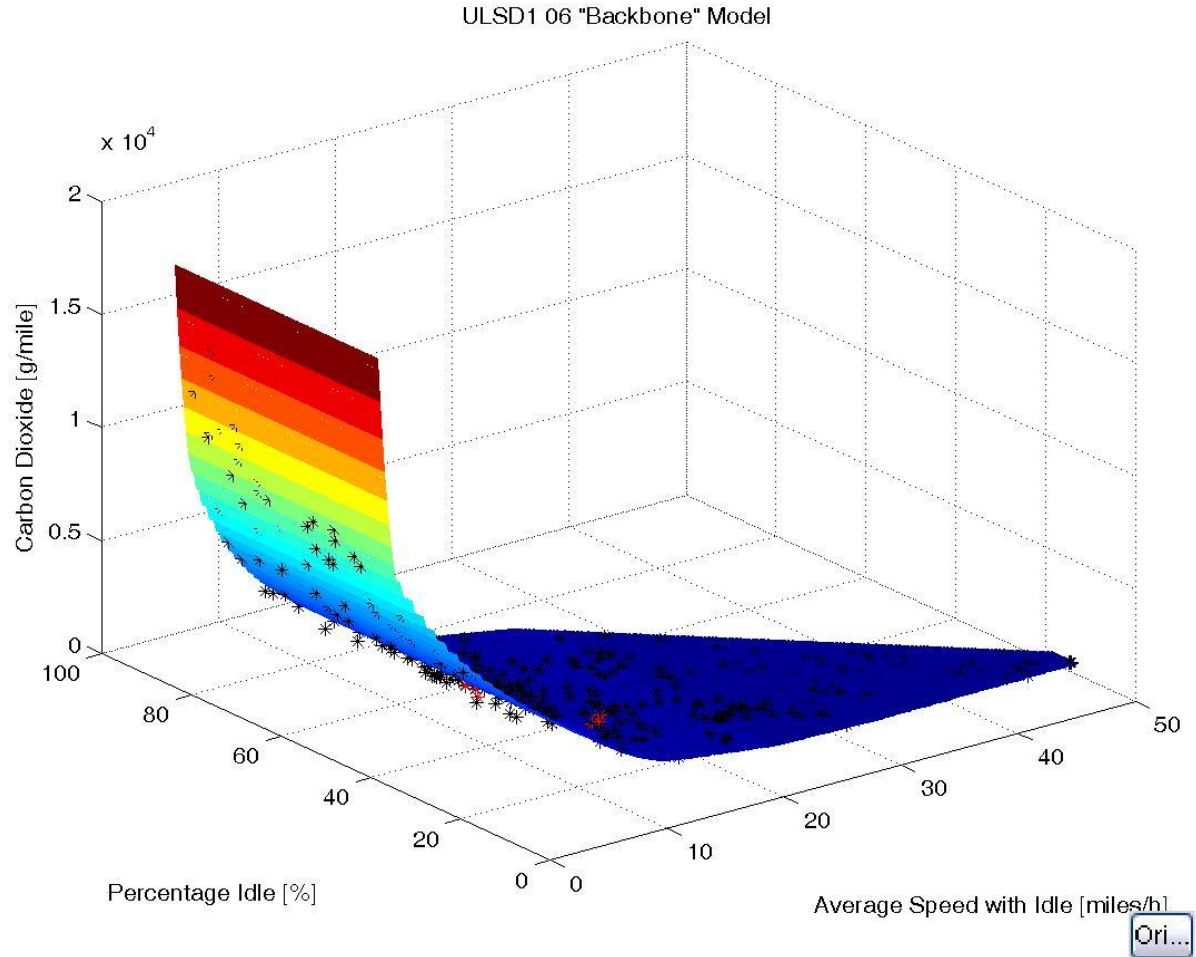

Figure C.9: Average speed \& PI backbone for $\mathrm{CO}_{2}$ 


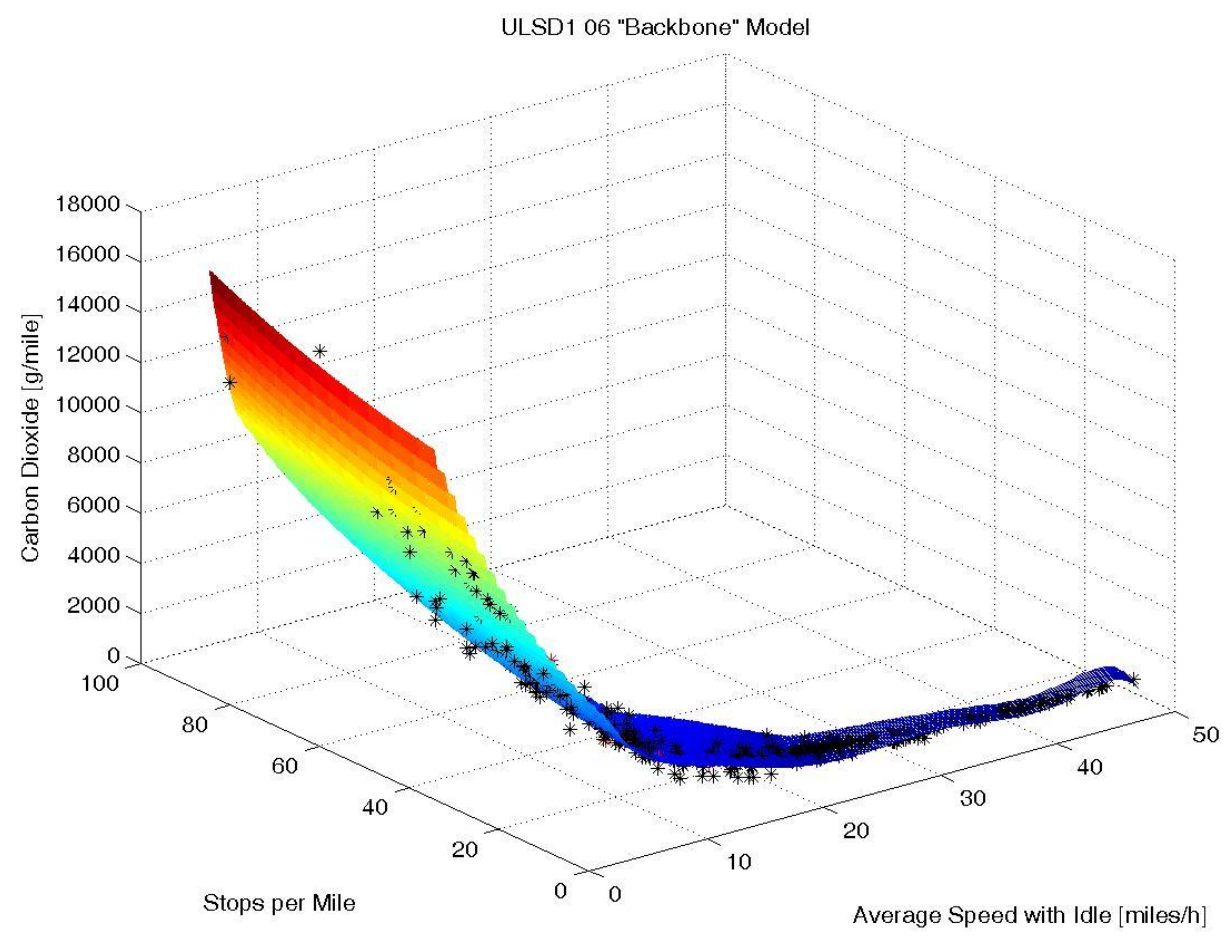

Figure C.10: Average speed \& stops per mile backbone for $\mathrm{CO}_{2}$

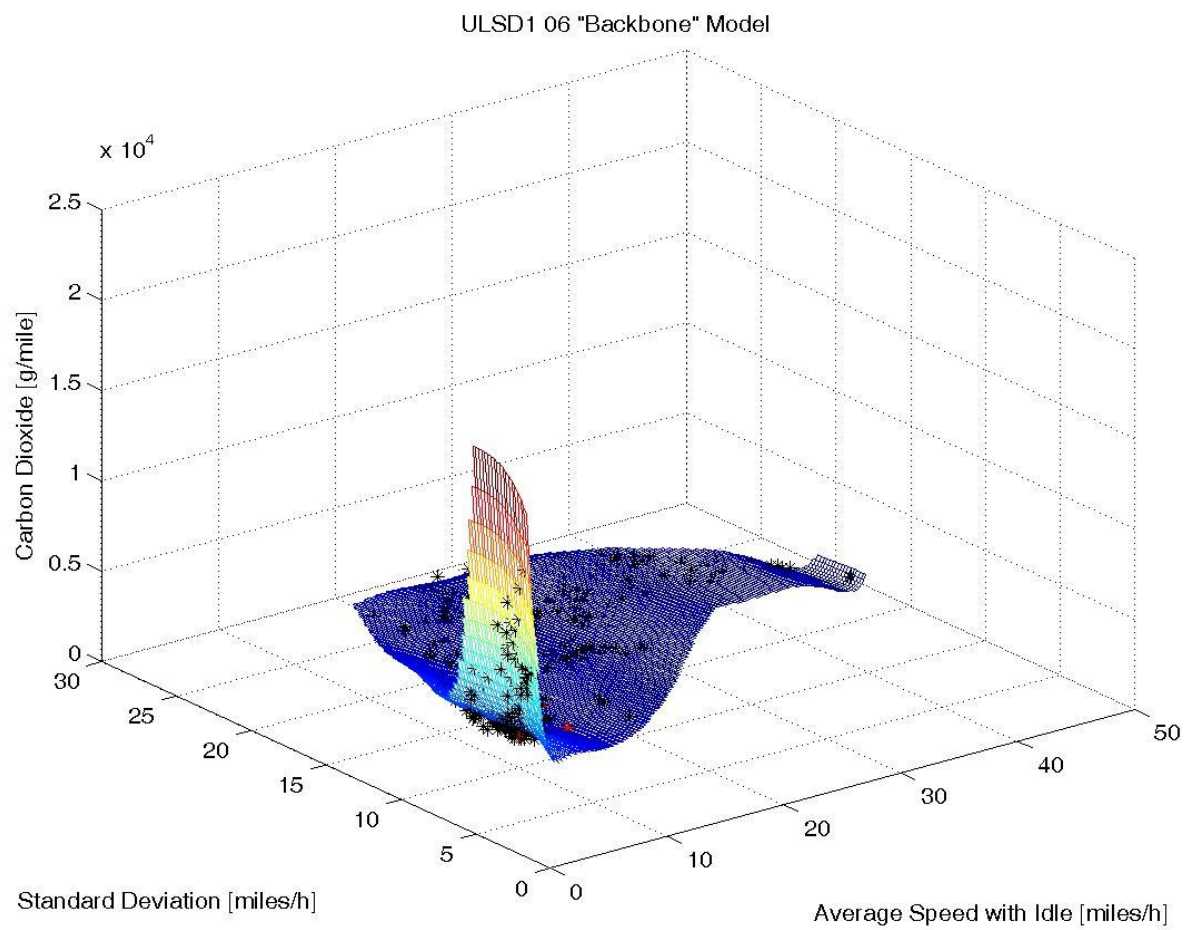

Figure C.11: Average speed \& STD backbone for $\mathrm{CO}_{2}$ 


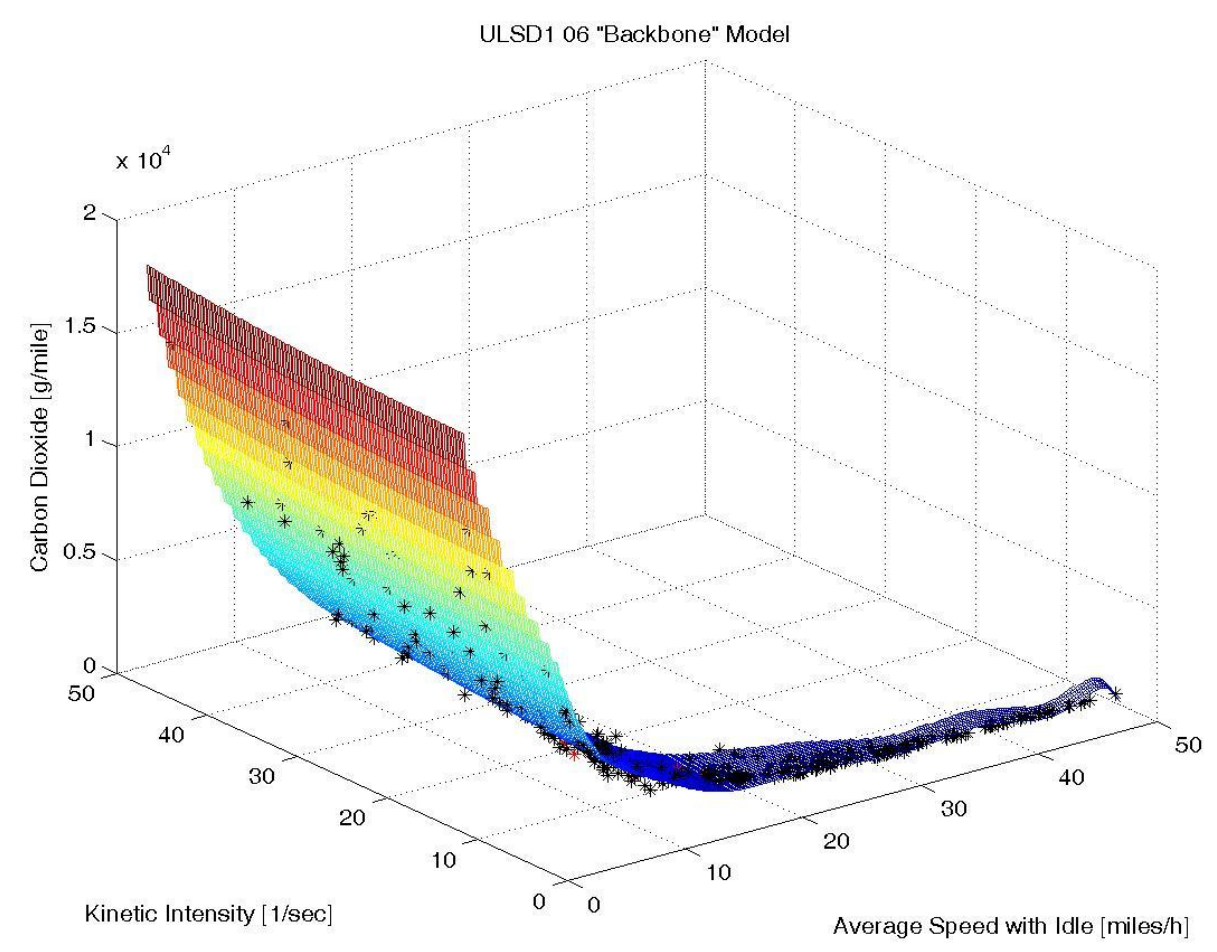

Figure C.12: Average speed \& $\mathrm{KI}$ backbone for $\mathrm{CO}_{2}$

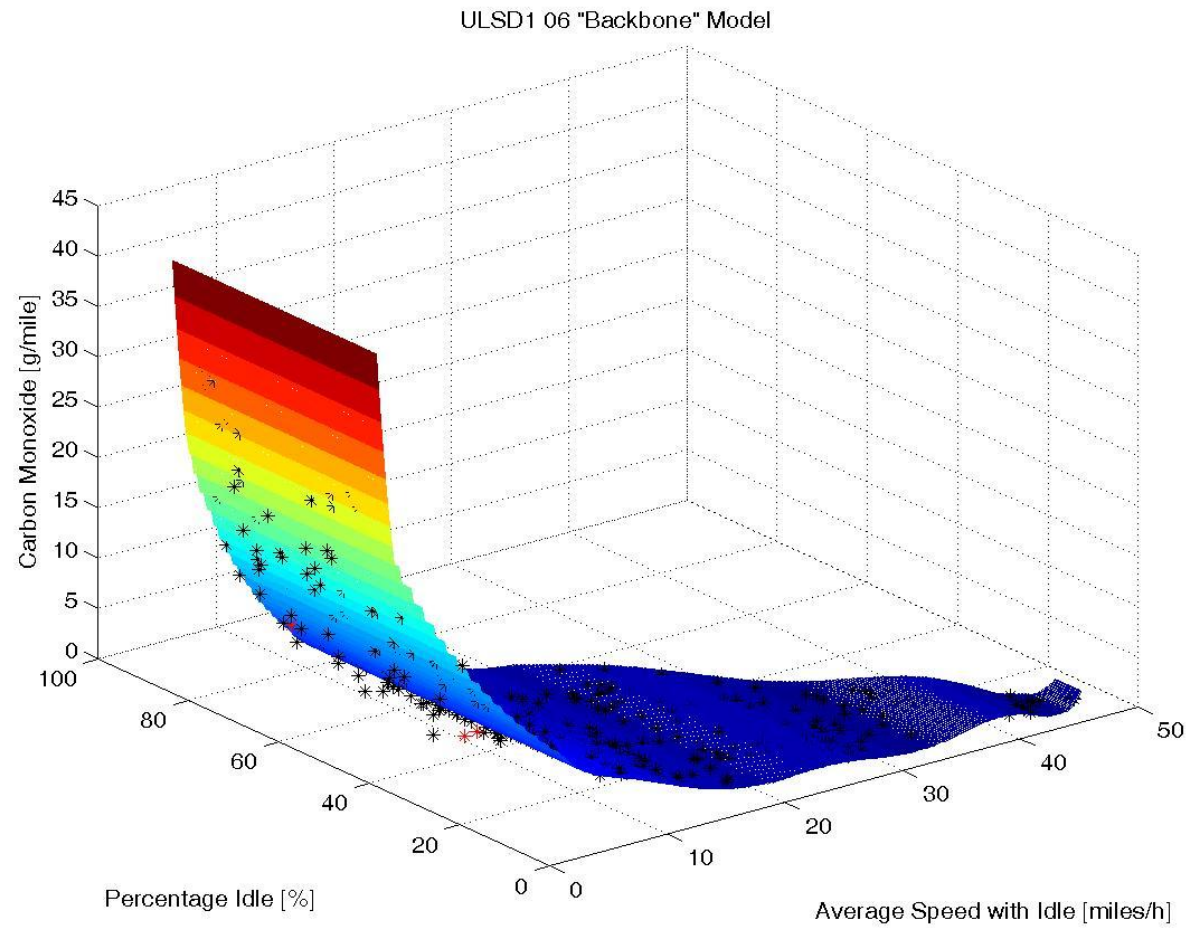

Figure C.13: Average speed \& PI backbone for CO 


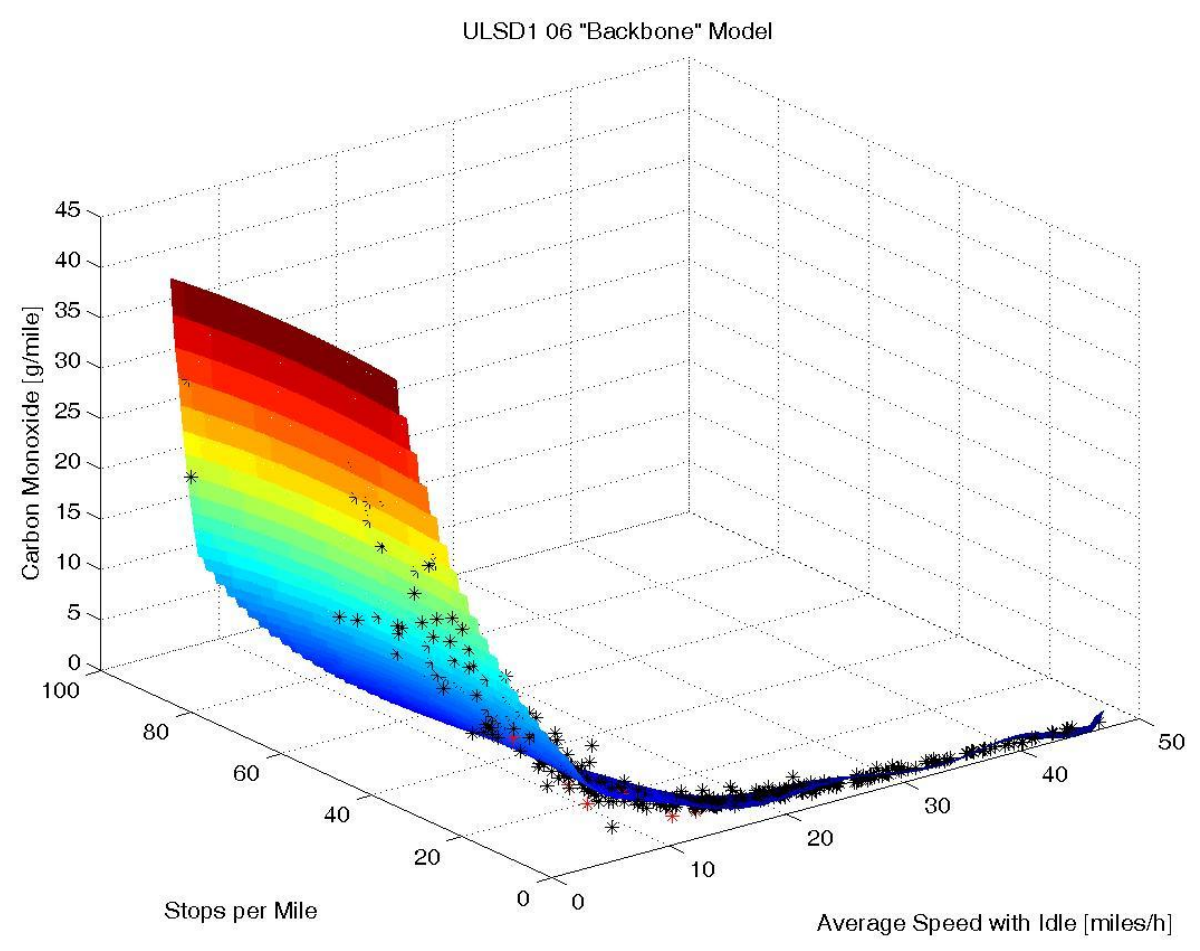

Figure C.14: Average speed \& stops per mile backbone for $\mathrm{CO}$

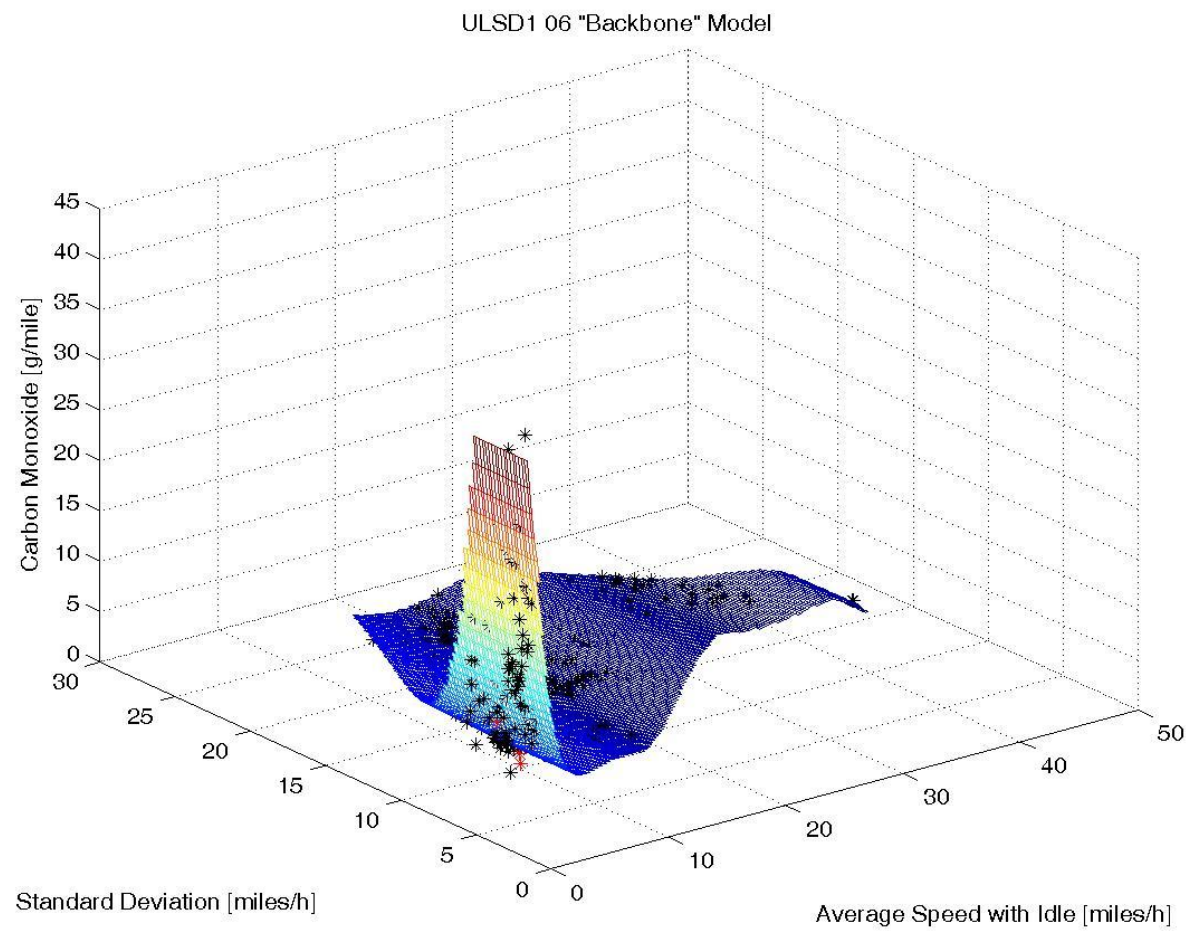

Figure C.15: Average speed \& STD backbone for CO 


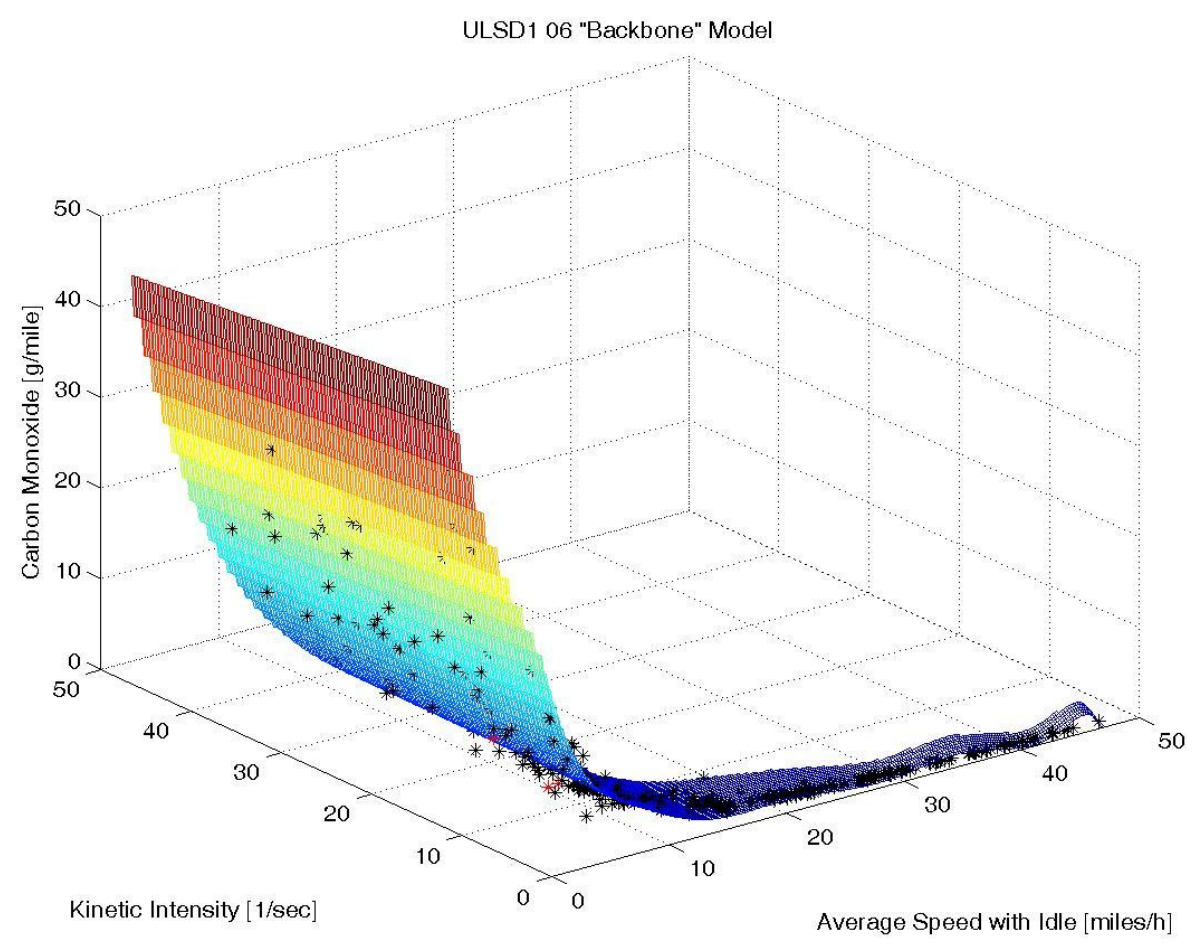

Figure C.16: Average speed \& KI backbone for $\mathrm{CO}$

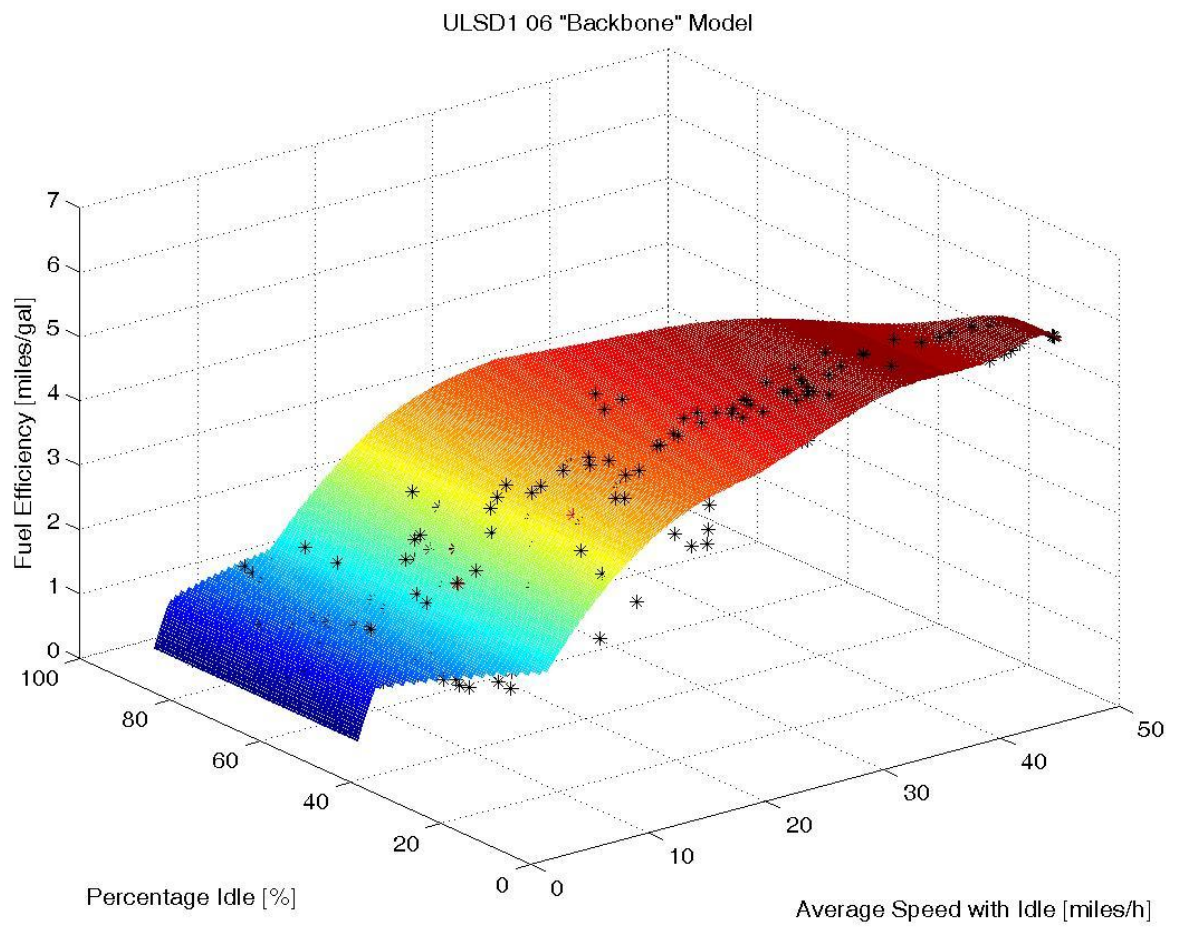

Figure C.17: Average speed \& PI backbone for Fuel Efficiency 


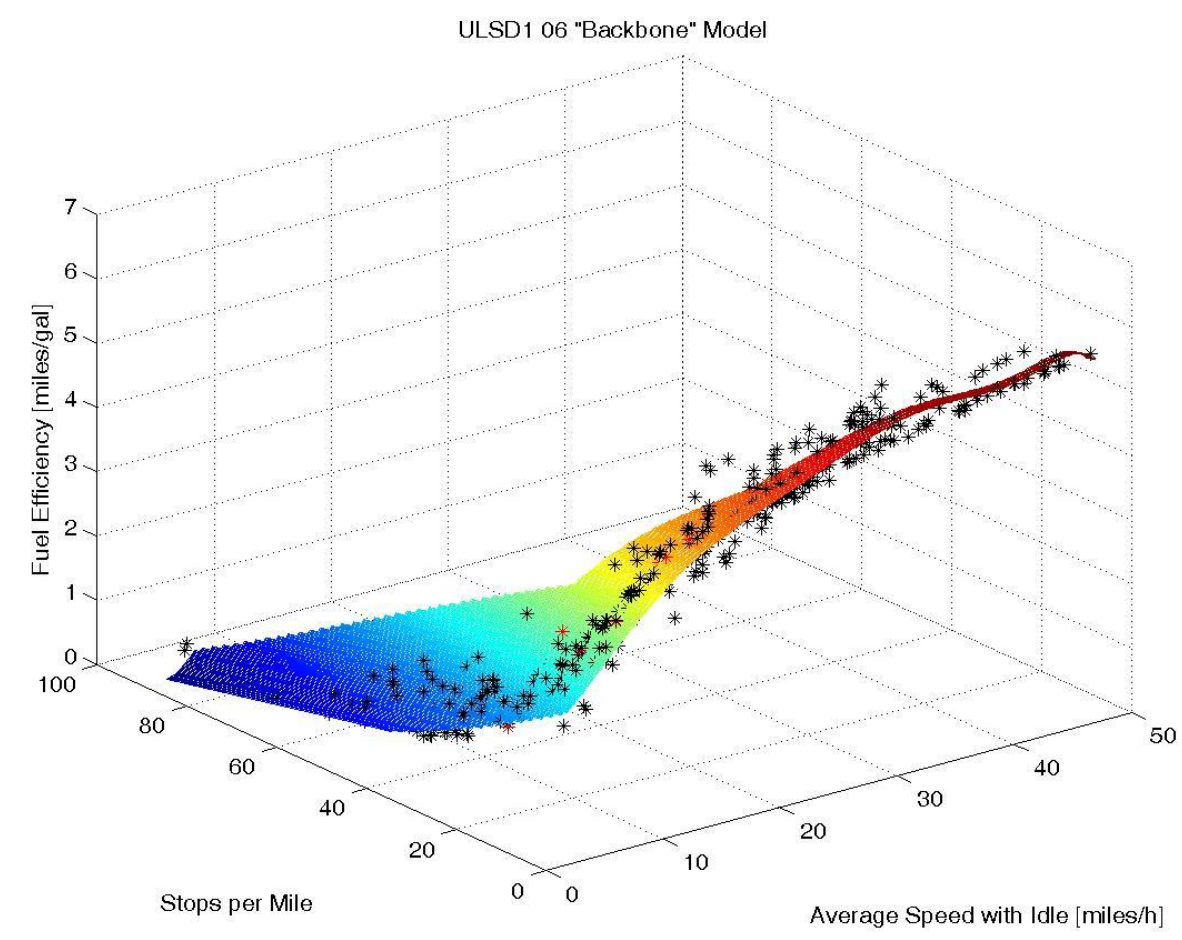

Figure C.18: Average speed \& stops per mile backbone for Fuel Efficiency

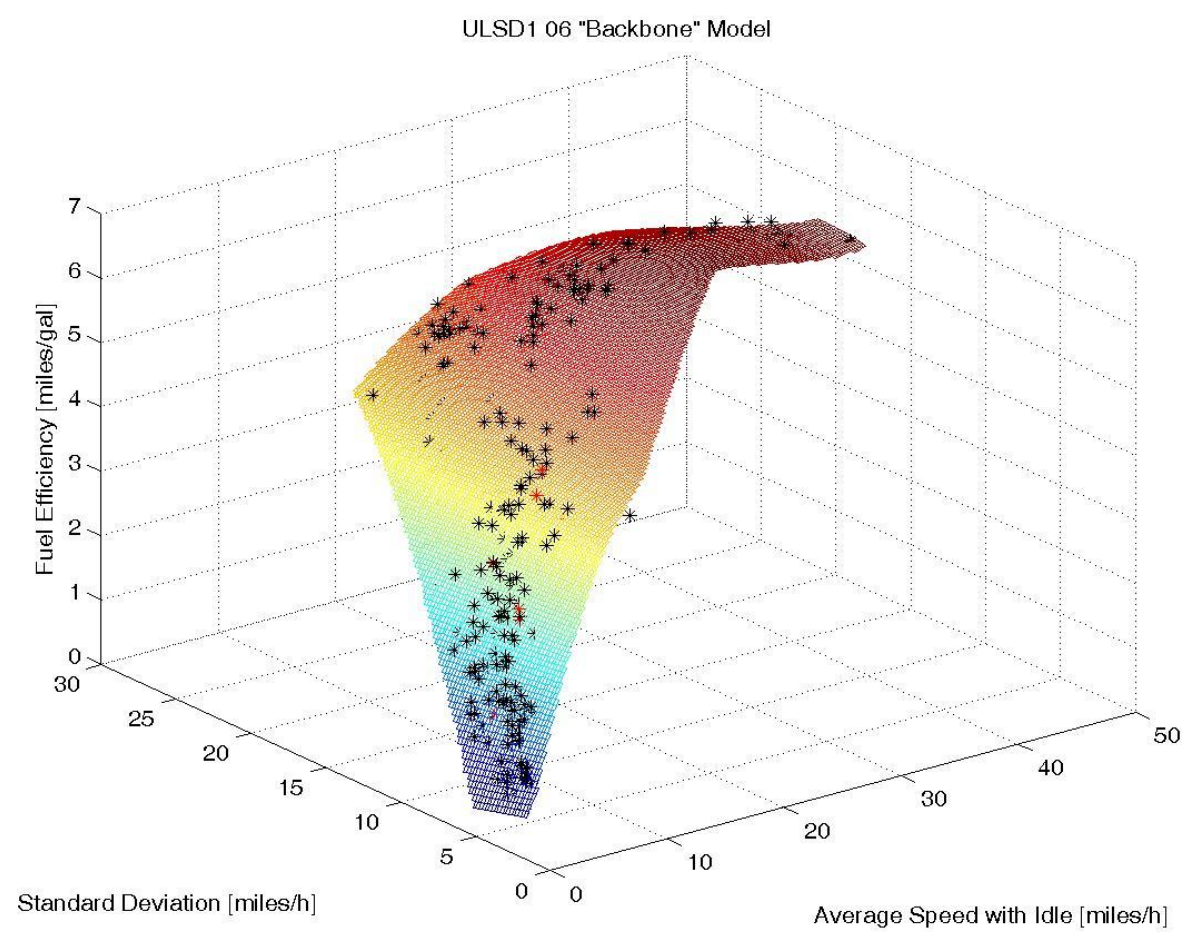

Figure C.19: Average speed \& STD backbone for Fuel Efficiency 


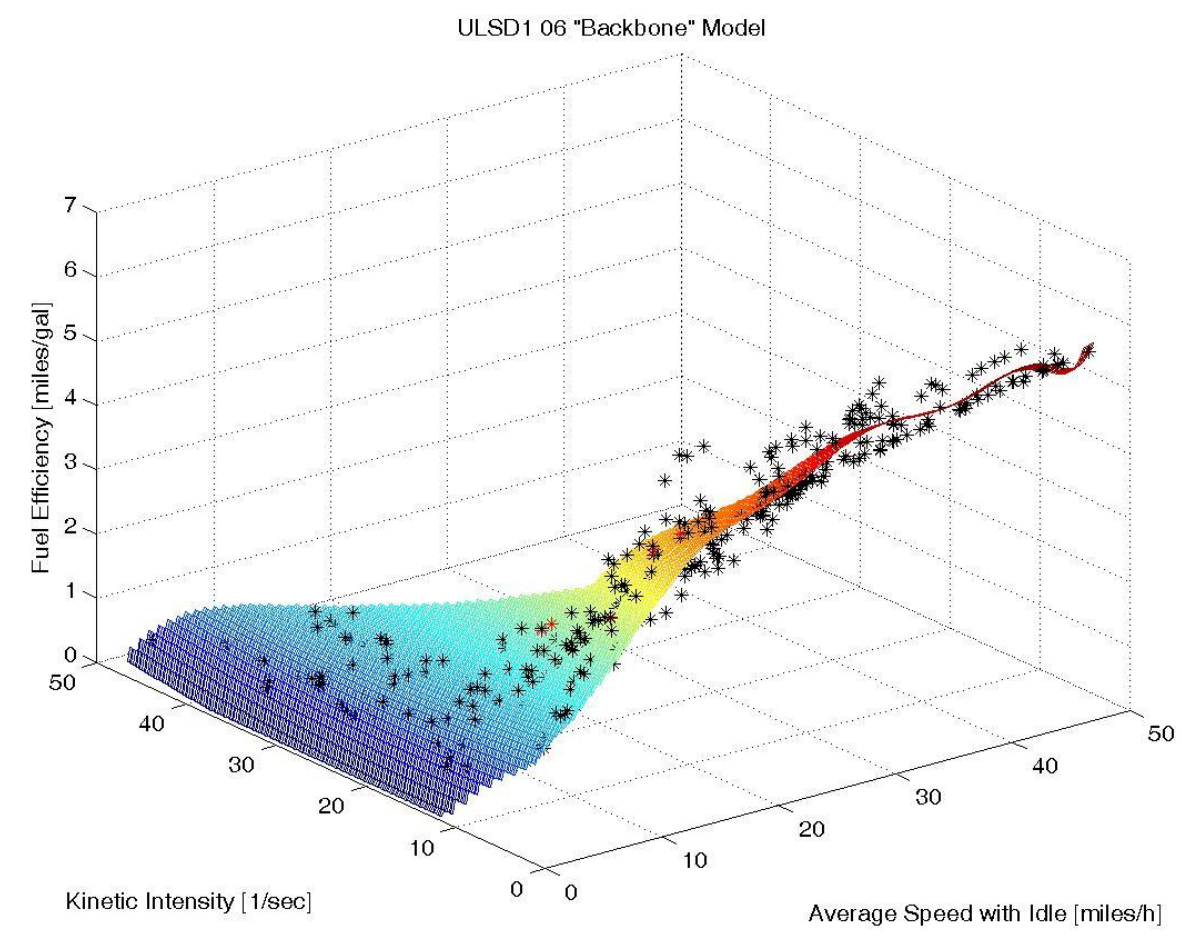

Figure C.20: Average speed \& KI backbone for Fuel Efficiency

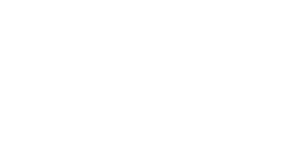

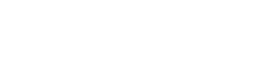

